\title{
Misregistration sensitivity in clustered-dot color halftones
}

\author{
Basak Oztan \\ University of Rochester \\ Department of Electrical and Computer Engineering \\ Rochester, New York 14627-0216 \\ E-mail: basak.oztan@ rochester.edu \\ Gaurav Sharma \\ University of Rochester \\ Department of Electrical and Computer Engineering \\ and \\ Department of Biostatistics and Computational Biology \\ Rochester, New York 14627-0126
}

\author{
Robert P. Loce \\ Xerox Research Center Webster \\ Xerox Corporation \\ Webster, New York 14580
}

\begin{abstract}
$\overline{\text { Abstract. Halftoned separations of individual colorants, typically }}$ cyan, magenta, yellow, and black, are overlaid on a print substrate in typical color printing systems. Displacements between these separations, commonly referred to as "interseparation misregistration", can cause objectionable color shifts in the prints. We study this misregistration-induced color shift for periodic clustered-dot halftones using a spatiospectral model for the printed output that combines the Neugebauer model with a periodic lattice representation for the individual halftones. Using Fourier analysis in the framework of this model, we obtain an analytic characterization for the conditions for misregistration invariance in terms of colorant spectra, periodicity of the individual separation halftones, dot shapes, and misregistration displacements. We further exploit the framework in a hybrid analytical-numerical simulation that allows us to obtain quantitative estimates of the color shifts due to misregistration, thereby providing a characterization for these shifts as a function of the optical dot gain, halftone periodicities, spot shapes, and interseparation misregistration amounts. We present simulation results that demonstrate the impact of each of these parameters on the color shift and demonstrate qualitative agreement between our approximation and experimental data. (c) 2008 SPIE and IS\&T. [DOI: 10.1117/1.2917517]
\end{abstract}

\section{Introduction}

*Halftoning is a method of encoding a continuous tone (contone) image using a reduced number of quantization levels, typically two, such that at normal viewing distances,

\footnotetext{
*Tis paper is available online as an open-access article, with color versions of several of the figures. In particular, Figs. $2,5,7,8$, and 17 may be difficult to interpret without color; print readers should refer to the online version at http:// SPIEDigitalLibrary.org for these figures.

Parts of this work were presented at the 2005 and 2006 Society for Imaging Science and Technology (IS\&T)/SPIE Electronics Imaging Symposiums ${ }^{1,2}$ and at the Institute of Electrical and Electronic Engineers (IEEE) International Conference on Image Processing $2007 .^{3}$

Paper 07147R received Jul. 19, 2007; revised manuscript received Oct. 25, 2007; accepted for publication Nov. 14, 2007; published online Jun. 5, 2008; corrected Jun. 9, 2008.

$1017-9909 / 2008 / 17(2) / 023004 / 30 / \$ 25.00$ @ 2008 SPIE and IS\&T.
}

the perception of the printed or displayed halftone image closely approximates that of the contone image. Color halftoning is used in most printing processes for the production of hardcopy prints. The halftoning operation is applied to the colorant image separations or channels, which usually correspond to the cyan $(\mathrm{C})$, magenta $(\mathrm{M})$, yellow $(\mathrm{Y})$, and black $(\mathrm{K})$ colorants. The halftoning operation produces a bilevel output for each separation and the printing process prints these colorant image separations in overlay on a white substrate such as paper. ${ }^{4,5}$ Halftoned images exercise only two extremes of a printing device's response corresponding to on/off states that represent a saturated amount of colorant or no colorant, respectively. Through process controls, it is easier to maintain these extremes in a stable condition over time, whereas, maintaining the stability of the printer response at intermediate levels is much more demanding. For a number of printing technologies, halftoning thus enables the production of prints that are relatively stable from print to print in color and tones and low in noise.

In an idealized, noninteracting, printing process, the halftoning algorithm for each colorant separation could be designed independently. ${ }^{6,7}$ In actual practice, however, there are interactions among the colorant separations that must be considered jointly in order to provide acceptable image quality. The interactions are best understood by considering that the light reflected from the paper to the observer is transmitted through the layer of toner or ink of each colorant separation. At a given point on the printed image, the resulting transmittance for each individual wavelength of light is, to first-order, the multiplication of transmittances from each colorant. For any wavelength that is absorbed by more than one colorant, this multiplicative phenomenon produces an interaction that varies spatially 
according to the halftone pattern produced by the halftoning algorithm. The spatial implications can be understood by recognizing that sum and difference frequency components are produced in the Fourier spectrum [i.e., a spatial Fourier spectrum per wavelength in the (visible) electromagnetic spectrum] via the convolution property of the Fourier transform [The convolution theorem (see Ref. 8, p. 108) states that the Fourier transform of the convolution/ multiplication of two signals is the multiplication/ convolution of their Fourier transforms]. These additional spatial frequency components could appear as undesirable texture or moiré in the printed image.

Due to the halftone interactions just described, most color halftoning methods in practice use halftone image structures that are designed to minimize the negative visual impact of low spatial frequency components that are the sum or difference of frequency components of the individual halftone separation images. A component at zero spatial frequency (commonly referred to as the "DC component") that arises from the sum or difference of other nonzero frequency components has, however, often not been considered undesirable. One reason for this apparent anomaly is that the color control of the printer eliminates the impact of a fixed DC component through the process used to map desired color values to printer CMYK combinations. However, the color response of a printer at DC (and in fact at any frequency) depends not only on the frequency components in the individual separation halftones but also on their relative phase. Spatial misregistration between the halftone colorant separations corresponds to a change in relative phase and can, therefore, alter the value of the DC term, which manifests as a color shift. Some amount and orientation of misregistration between halftone image separations is unavoidable due to various operations within a printing process such as mechanical paper transport, paper shrinkage, and misalignment of imagers. In the present work, we investigate this interseparation misregistration-induced color shift by examining the zero spatial frequency component produced in the overlay. Our goal is to investigate the impact of misregistration both analytically and by means of a simulation framework.

The preceding discussion is applicable to almost all halftoning methods that process colorant separations independently. Several techniques have been proposed for the halftoning of individual separations (or equivalently monochrome images) that fall under one of the following three categories: ${ }^{5}(1)$ point processes, (2) neighborhood algorithms, and (3) iterative methods. The characteristics of the printing technology, specific application requirements, and computational complexity are some of the common factors that impact the choice of a halftoning technique. Due to their stability and predictability, clustered-dot halftones are commonly used in the two primary methods of high volume printing: lithography and xerography. Clustered-dot halftoning evolved from Talbot's original photographic screening process ${ }^{9}$ and, for digital imagery, is typically accomplished by pixel-by-pixel thresholding against a periodic halftone threshold array. Conventional clustered-dot halftones are considered amplitude modulated (AM) signals in the sense that different gray levels are reproduced by varying the size of halftone spots while keeping their periodicity constant. In the present paper, we re- strict our attention to methods used for xerographic and offset printers and, thus, consider only clustered-dot halftones in our analysis of color variation with interseparation misregistration.

The remainder of this paper is organized as follows. Section 2 reviews the literature on this problem and connects it to our contributions. In Sec. 3, we develop a characterization of halftone color sensitivity to interseparation misregistration using Fourier analysis in a lattice framework. In Sec. 4, the conditions under which the average color is invariant to displacement misregistration are described. Next, in Sec. 5, we incorporate an analytic model for the halftone screens in our lattice framework to develop a numerical model for quantitative estimation of color shifts due to misregistration in clustered-dot color halftones. We present results from the model along with comparisons to experimental data in Sec. 7. A discussion of the additional practical implications and extensions is included in Sec. 8. Based on that development, we also propose a metric for evaluating misregistration sensitivity of twocolorant halftone configurations. Finally, Sec. 9 summarizes the main conclusions.

\section{Related Work}

The average spectrum of a halftone image may be modeled (to first-order) using the Neugebauer equations. ${ }^{10,11}$ Using these equations, we can readily see that if the areas of overlap between the colorant separations can be modeled as statistically random, that is, satisfy the Demichel equations, ${ }^{12}$ the average spectrum is independent of the interseparation alignment. Therefore, randomization of the interseparation overlaps eliminates the problem of misregistration-induced color shift in color halftones. With this motivation, rotated halftone screens, in which the halftones for different separations are rotated relative to each other, are commonly used in practice. Though alternatives to the Demichel equations have been proposed for nonrotated halftone configurations, ${ }^{13,14}$ the validity of the Demichel equations for the common rotated screen configurations has not received much attention until recently.

Rogers ${ }^{15}$ examined the validity of Demichel equations for the superposition of two halftone screens with circular halftone spots, where each screen has two orthogonal frequency vectors and the four frequency vectors for the screens have equal magnitudes. In this scenario, he demonstrated that the equations hold only for certain angular separations between the screens. Rogers achieved this result by deriving an expression for the neighbor distribution of halftone spots and determining the conditions under which this distribution is statistically random.

Amidror and Hersch ${ }^{16}$ extend the work to the superposition of an arbitrary number of screens. They present a general proposition that characterizes the failure of the Demichel equations in the Fourier domain by a singular configuration of frequency vectors [We provide a precise mathematical definition of the term singular in the next section]. Using computer simulations on a high resolution pixel grid, they numerically demonstrate the validity of the proposition. In particular, they verify that the conventional 30-deg angular separation equifrequency CMK halftone configuration (see Ref. 6, pp. 339-341) used in lithographic printing is not invariant to color misregistration. In related 


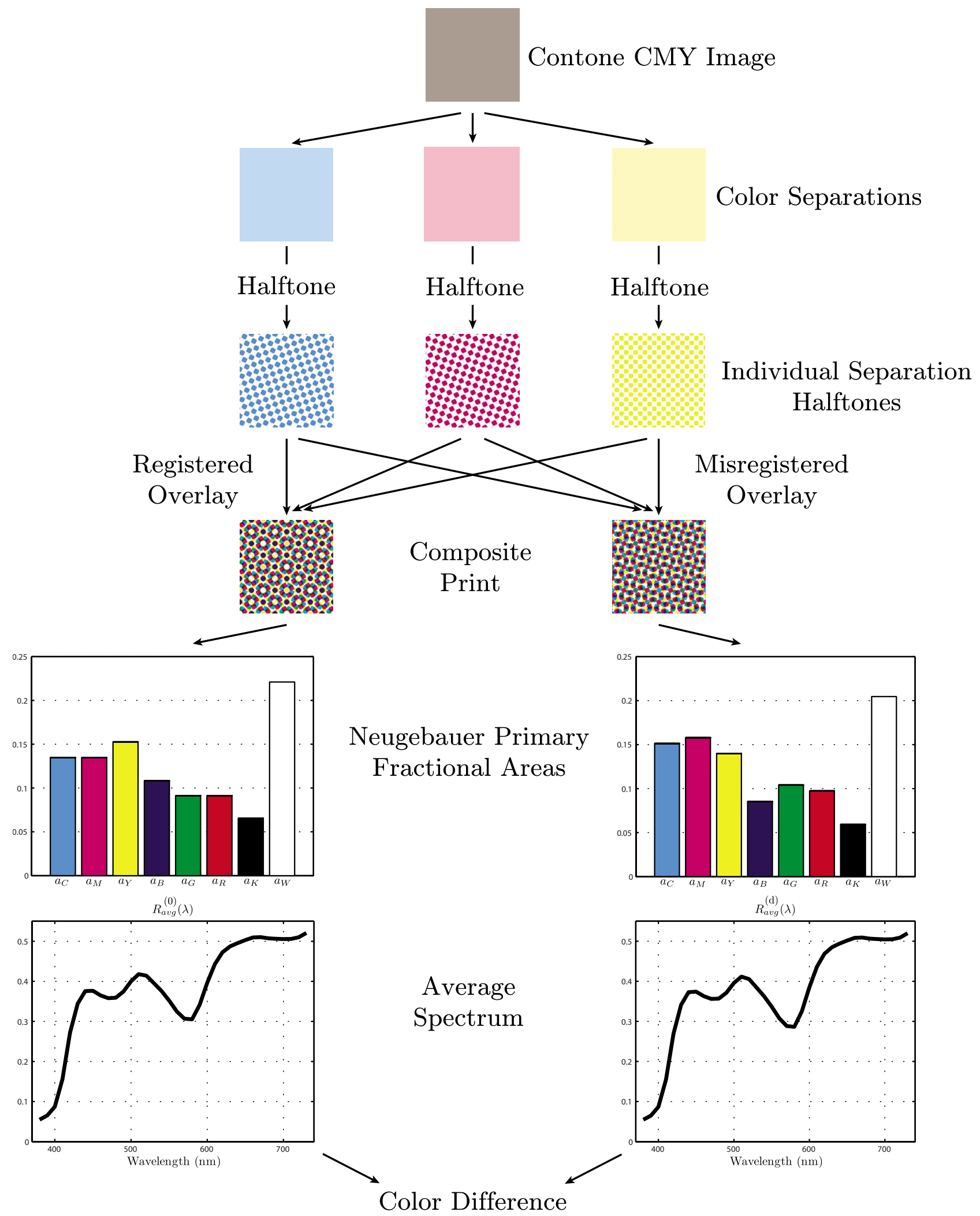

Fig. 1 Modeling framework for analyzing the effect of interseparation misregistration on the color (average reflectance spectrum) of periodic clustered-dot halftone prints. (Color online only.) 
work, ${ }^{17}$ they also consider the stability of the rosette structure, also known as microstructure, resulting from the overlap of multiple screens and demonstrate that the microstructure is sensitive to layer misregistration for singular configurations and insensitive for nonsingular configurations.

In the first part of our work, we build on the foundations of Rogers ${ }^{15}$ and Amidror and Hersch. ${ }^{16,17}$ We cast the general $K$-screen superposition problem in a lattice framework that has previously been used for the frequency analysis of halftone superpositions. Each halftone separation is modeled as a periodic function with periodicity determined by a two-dimensional (2D) lattice. Using Fourier analysis in our lattice framework, we obtain mathematical expressions for the average halftone color (spectrum) as a function of screen periodicities, halftone spots, interseparation screen displacements, and Neugebauer primary spectra. The analysis provides a comprehensive framework for understanding the conditions for color insensitivity to displacement misregistration. Conventionally, it is believed that misregistration insensitivity is achieved if either the colorants have nonoverlapping absorption bands, ${ }^{6}$ or if the halftone periodicities meet a "nonsingularity" condition. ${ }^{16}$ In addition to validating these known criteria, our analysis reveals additional situations under which insensitivity to misregistration is achievable despite these conditions being violated. In particular, we demonstrate that in some scenarios, the halftone spot functions may provide invariance to misregistration. The analysis also reveals the existence of nontrivial (different from an integral number of lattice periods) displacements for which the invariance holds.

In the second part of our work, we address the quantitative estimation of the color shift induced by misregistration. For this purpose, we use the Neugebauer model in a semianalytic simulation that exploits the analysis from the first part to obtain spatial domain expressions for the average spectrum of the print expressed in terms of colorant overlap areas. The latter are computed analytically for common dot shapes. When combined with measured Neugebauer primary spectra, these allow for a quantification of the color shift for different misregistrations. Prior work in this area has also been based on the Neugebauer model with estimation of the area coverages for the Neugebauer primaries performed either experimentally by using a planimeter for the dot-on-dot geometry, ${ }^{18}$ or through computer simulations, where halftone images are generated on a high resolution digital grid and areas computed by pixel-counting methods. ${ }^{1,19,16}$ The experimental approach has only been applied in a limited context due to measurement challenges. The pixel-counting simulation approaches become quite memory intensive for certain configurations and can also suffer from a limitation in accuracy due to the use of a finite grid. Our semianalytic methodology allows the model to operate without the computationally expensive high resolution simulation, thereby enabling evaluation of numerous screen configurations. Comparison of the framework against experimental data with independent measurement of misregistration demonstrates good qualitative agreement.

\section{Color Halftone Misregistration Analysis Framework}

For our analysis, we assume that color printing is accomplished by halftoning $K$ individual colorant separations, where $K=4$ for the typical CMYK scenario, and printing these in overlay. In Fig. 1, we schematically illustrate the overall process for a typical clustered-dot color halftoning with CMY colorants. We model individual colorant clustered-dot halftones in terms of a lattice that represents their periodicity and a spot function that represents the shape of the halftone dots. The average color for the printed overlay of these halftones is obtained by using the spectral Neugebauer model. ${ }^{10}$ The model computes the spatially averaged reflectance for the print as a weighted average of reflectances of all possible overlays of the colorants on the substrate, where the overlays are referred to as the Neugebauer primaries and the weights correspond to the fractional areas of the Neugebauer primaries. A change in these fractional areas due to interseparation misregistration is the primary source of color shifts when the colorants have overlapping absorption bands. We therefore derive our model in this framework and obtain expressions for the average reflectance spectrum and the fractional areas, which we use in turn to characterize the conditions under which the average spectrum is invariant to displacement misregistration.

\subsection{Individual Colorant Halftones}

A halftone image $h_{k}(\mathbf{x})$ generated for the $k$ 'th colorant plane of a constant gray-level contone image can be modeled as the convolution of a planar lattice $\boldsymbol{\Lambda}_{k}$ and a binary halftone spot function $s_{k}(\mathbf{x}),{ }^{20,21}$ where $\mathbf{x}=[x, y]^{T}$ represents the spatial coordinates. $\boldsymbol{\Lambda}_{k}$ represents the $2 \mathrm{D}$ periodicity of the $k^{\prime}$ th halftone separation and is mathematically defined as ${ }^{22}$

$\boldsymbol{\Lambda}_{k}=\left\{\mathbf{V}_{k} \mathbf{n}_{k} \mid \mathbf{n}_{k} \in \mathbb{Z}^{2}\right\}$,

where $\mathbb{Z}$ denotes the set of integers and $\mathbf{V}_{k}=\left[\mathbf{v}_{1}^{k} \mathbf{v}_{2}^{k}\right]$ is a $2 \times 2$ real-valued matrix, with two $2 \times 1$ linearly independent vectors $\mathbf{v}_{1}^{k}=\left[v_{x_{1}}^{k}, v_{y_{1}}^{k}\right]^{T}$ and $\mathbf{v}_{2}^{k}=\left[v_{x_{2}}^{k}, v_{y_{2}}^{k}\right]^{T}$ as its columns. Thus, $\boldsymbol{\Lambda}_{k}$ is the (discrete) set of all integer linear combinations of the vectors $\mathbf{v}_{1}^{k}$ and $\mathbf{v}_{2}^{k}$ in $\mathbb{R}^{2}$. The vectors $\mathbf{v}_{1}^{k}$ and $\mathbf{v}_{2}^{k}$ represent a basis for the lattice $\boldsymbol{\Lambda}_{k}$ and for any point $\mathbf{V}_{k} \mathbf{n}_{k}$ in the lattice, the vector $\mathbf{n}_{k}=\left[n_{k x}, n_{k y}\right]^{T}$ is the representation of the point in the lattice with respect to the basis $\mathbf{V}_{k}$.

The halftone spot function $s_{k}(\mathbf{x})$ is confined within a unit cell of $\boldsymbol{\Lambda}_{k}$, denoted by $\mathcal{U}_{k}$, and takes values 1 or 0 corresponding to the situation that ink $k$ is, or is not, deposited at the position $\mathbf{x} . h_{k}(\mathbf{x})$ can accordingly be written as

$h_{k}(\mathbf{x})=s_{k}(\mathbf{x}) * \sum_{\mathbf{n}_{k}} \delta\left(\mathbf{x}-\mathbf{V}_{k} \mathbf{n}_{k}\right)$

where $\delta(\mathbf{x})$ is the Dirac delta function. Displacement misregistration of the $k^{\prime}$ th separation by the vector $\mathbf{d}_{k}$ $=\left[\Delta x_{k}, \Delta y_{k}\right]^{T}$ is readily incorporated in this representation by replacing $s_{k}(\mathbf{x})$ with $s_{k}\left(\mathbf{x}-\mathbf{d}_{k}\right)$, where $\Delta x_{k}$ and $\Delta y_{k}$ are the registration errors along the $x$ and $y$ axes, respectively. The halftone separation $h_{k}(\mathbf{x})$ can accordingly be written as 


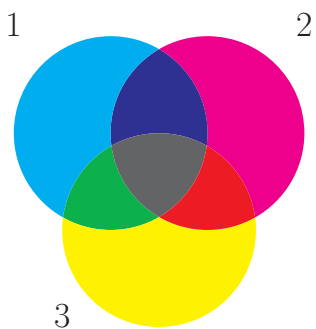

(a) Colorant overlay
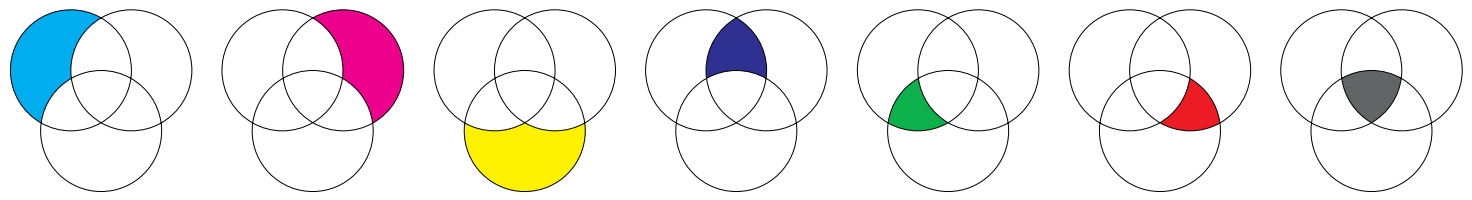

$a_{100}$

$a_{010}$

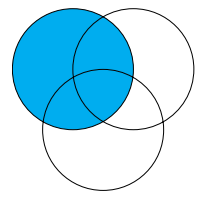

$\beta_{1}$

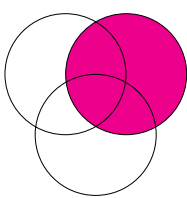

$\beta_{2}$ $a_{001}$

$a_{110}$

$a_{101}$

$a_{011}$

$a_{111}$

(b) Neugebauer primary area $a_{\mathbf{c}}$

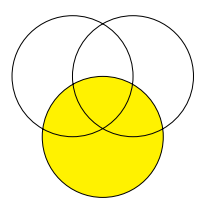

$\beta_{3}$

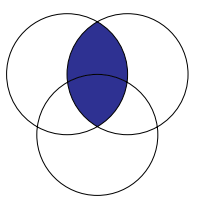

$\beta_{12}$

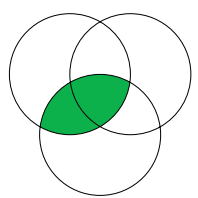

$\beta_{13}$

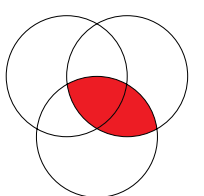

$\beta_{23}$

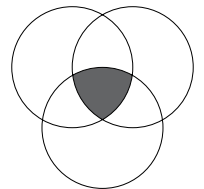

$\beta_{123}$

(c) Colorant overlap area $\beta_{\mathbf{k}(\mathbf{c}}$

Fig. 2 Illustration of the relation between the Neugebauer primary areas $a_{\mathrm{c}}$ and colorant overlap areas $\beta_{\mathbf{k}(\mathbf{c})}$ for $K=3$ with CMY colorants. (Color online only.)

$h_{k}^{\left(\mathbf{d}_{k}\right)}(\mathbf{x})=s_{k}\left(\mathbf{x}-\mathbf{d}_{k}\right) * \sum_{\mathbf{n}_{k}} \delta\left(\mathbf{x}-\mathbf{V}_{k} \mathbf{n}_{k}\right)$.

\subsection{Spectral Neugebauer Model}

On a color print, these multiple halftone image separations are overlaid, typically producing all possible $2^{K}$ overlays of $K$ colorants. The colors associated with each of the $2^{K}$ overlays are referred to as the Neugebauer primaries. Using the Yule-Nielsen (YN) modified Neugebauer model, ${ }^{10,11,23}$ the average spectrum of the printed halftone is

$R_{\text {avg }}(\lambda)=\left(\sum_{i=0}^{2^{K}-1} a_{i} R_{i}^{\frac{1}{\gamma}}(\lambda)\right)^{\gamma}$,

where $a_{i}$ and $R_{i}(\lambda)$ are the fractional area coverage and the spectral reflectance of the $i$ 'th Neugebauer primary, respectively, and $\gamma$ is the empirical YN correction factor. This factor accounts for optical dot gain, that is, the scattering of the absorbed light within the paper due to which halftone spots appear larger than their physical size on the paper. Paper quality, spectral characteristics of the colorants, halftone periodicities, and spot shapes are some of the elements that affect optical dot gain. Generally $1 \leq \gamma \leq 2$ is accepted as physically meaningful, ${ }^{24,25}$ although, empirically, values of $y>2$ often provide better agreement with data, particularly for high frequency printers. ${ }^{26}$

To obtain expressions for the Neugebauer primary areas, we first represent these areas in terms of an alternative but equivalent (in the sense that either is obtainable from the other) set of areas that are more readily amenable to analysis. Figure 2 illustrates this alternate representation, which we describe next. For notational convenience, in this process, we index each of the $2^{K}$ possible combinations of the $K$ colorants by a $K$-bit binary index string $\mathbf{c}=c_{1} \ldots c_{K}$, where $c_{k}=1$ indicates the presence of the $k^{\prime}$ th colorant and $c_{k}=0$ its absence in the combination. Interpreting the string as the binary representation of a Neugebauer primary index between 0 and $2^{K}-1$, we can rewrite Eq. (4) as

$R_{\text {avg }}(\lambda)=\left(\sum_{c_{1}=0}^{1} \cdots \sum_{c_{K}=0}^{1} a_{c_{1} \ldots c_{K}} R_{c_{1} \cdots c_{K}}^{1 / \gamma}(\lambda)\right)^{\gamma}$.

Now if we denote by $\beta_{k}$ the fraction of the total area covered by the $k^{\prime}$ th colorant (which is possibly covered by additional colorants and, therefore, distinct from the area for the corresponding Neugebauer primary), we have

$\beta_{k}=\sum_{\left\{c_{1} \ldots c_{K} \mid c_{k}=1\right\}} a_{c_{1} \ldots c_{K}}$.

We extend this notation and use $\beta_{k_{1} \ldots k_{m}}$ to represent the fractional area covered by the colorants $k_{1} \ldots k_{m}$ (parts of which are also possibly covered by additional colorants), where $k_{1} \ldots k_{m}$ is a set of distinct colorant indices drawn from $\{1, \ldots, K\}$. For uniqueness, we consider only subscripts $k_{1} \ldots k_{m}$ indices arranged in ascending order, that is $k_{1}<k_{2}<\cdots<k_{m}$. Then, just as with the individual colorant areas, these fractional areas can be represented in terms of the Neugebauer primary areas as 


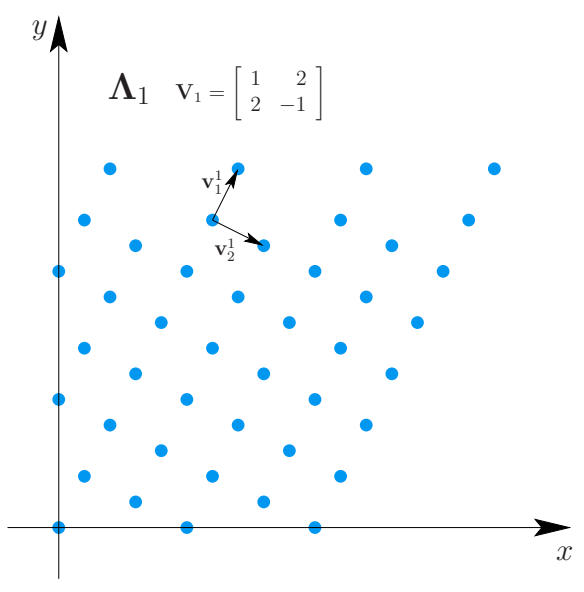

(a)

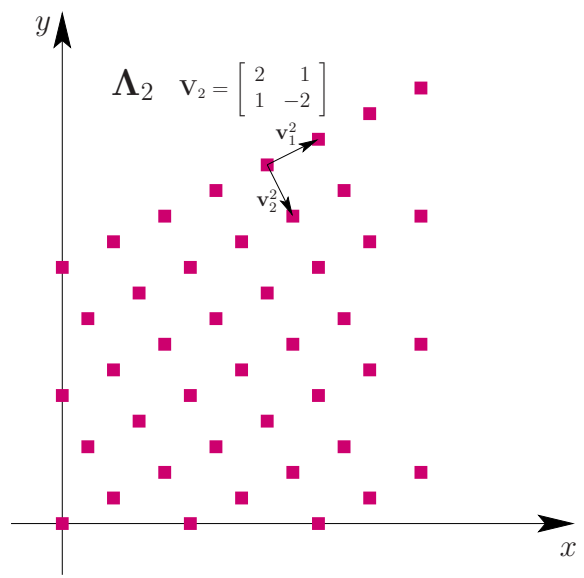

(b)

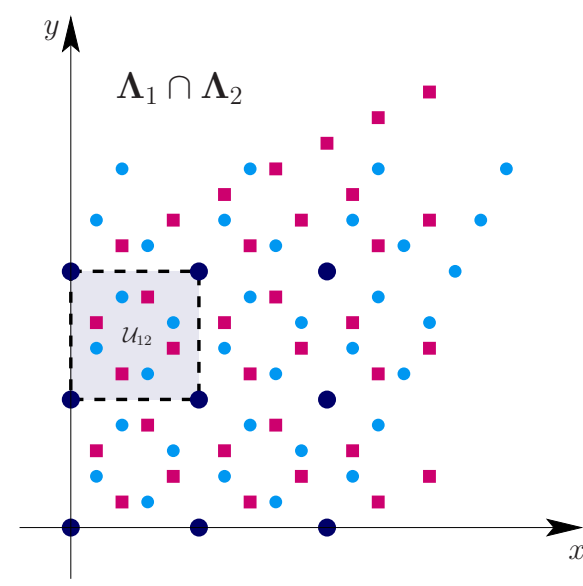

(c)

Fig. 3 Example of lattice intersection. Panels (a) and (b) show points on lattices $\Lambda_{1}$ and $\Lambda_{2}$, respectively. The larger blue circles in (c) show the intersection points of the overlaid lattices and the region inscribed by the dashed lines shows a unit cell of the intersection denoted by $\mathcal{U}_{12}$.

$$
\beta_{k_{1} \ldots k_{m}}=\sum_{\left\{c_{1} \ldots c_{K} \mid c_{k_{1}}=1, \ldots, c_{k_{m}}=1\right\}} a_{c_{1} \ldots c_{K}} .
$$

The above system of equations can be inverted to obtain an expression for the fractional areas of the primaries $a_{c_{1} \ldots c_{K}}$ in terms of the fractional areas $\beta_{k_{1} \ldots k_{m}}$ for the colorant combinations. One can see that $a_{1 \ldots 1}=\beta_{1 \ldots K}$ and

$a_{c_{1} \ldots c_{K}}=\left\{\begin{array}{c}1-\sum_{\mathbf{c} \in \mathcal{I}^{+}(\mathbf{c})} a_{\mathbf{c}} \quad \text { if } c_{1} \ldots c_{K}=0 \ldots 0, \\ \beta_{\mathbf{k}(\mathbf{c})}-\sum_{\mathbf{c} \in \mathcal{I}^{+}(\mathbf{c})} a_{\mathbf{c}} \quad \text { otherwise, }\end{array}\right.$

where the notation $\mathbf{k}(\mathbf{c})=k_{1} \ldots k_{m}$ denotes the string of indices $\left\{k_{l}\right\}_{l=1}^{m}$ for which $c_{k_{l}}$ is nonzero, and $\mathcal{I}^{+}(\mathbf{c})$ is the set of all indices that include the nonzero indices $c_{k_{l}}$ and at least one additional (distinct) nonzero index [Note that each element of $\mathcal{I}^{+}(\mathbf{c})$ denotes a combination of colorants that include the nonzero $m$ colorants indicated by $\mathbf{c}$ as a subset and at least one additional nonzero colorant]. The above relation [Eq. (8)] can also be seen from Fig. 2, which illustrates the relation between the areas $a_{\mathbf{c}}$ and $\beta_{\mathbf{k}(\mathbf{c})}$ for $K=3$ with CMY colorants.

Due to the equivalence of the Neugebauer primary fractional areas $a_{c_{1} \ldots c_{K}}$ to the colorant overlap areas $\beta_{\mathbf{k}(\mathbf{c})}$, invariance properties with respect to displacement misregistration established for one are applicable to the other. We therefore proceed by obtaining expressions for the areas $\beta_{\mathbf{k}(\mathbf{c})}$. Note that we adopt this approach because it is easier to directly obtain expressions for the terms $\beta_{\mathbf{k}(\mathbf{c})}$, as opposed to the Neugebauer primary areas $a_{\mathbf{c}}$.

\subsection{Fractional Areas of Colorant Combinations}

To compute $\beta_{\mathbf{k}(\mathbf{c})}$, consider the overlay of the halftones that constitute $\mathbf{k}(\mathbf{c})$. The function $h_{\mathbf{k}(\mathbf{c})}\left(\mathbf{x} ; \mathbf{d}_{\mathbf{k}(\mathbf{c})}\right)=\prod_{k \in \mathbf{k}(\mathbf{c})} h_{k}^{\left(\mathbf{d}_{k}\right)}(\mathbf{x})$

indicates the spatial locations covered by the colorants in $\mathbf{k}(\mathbf{c})$, taking a value 1 if $\mathbf{x}$ is covered by the colorants in $\mathbf{k}(\mathbf{c})$ and 0 otherwise, where $\mathbf{d}_{\mathbf{k}(\mathbf{c})}=\left[\mathbf{d}_{k_{1}}, \ldots, \mathbf{d}_{k_{m}}\right]$ are the displacement vectors of the individual separations that constitute $\mathbf{k}(\mathbf{c})$. The fractional area $\beta_{\mathbf{k}(\mathbf{c})}$ is the spatial average of $h_{\mathbf{k}(\mathbf{c})}\left(\mathbf{x} ; \mathbf{d}_{\mathbf{k}(\mathbf{c})}\right)$.

If each of the matrices $\mathbf{V}_{k_{j}}^{-1} \mathbf{V}_{k_{j}}$ has only rational numbers as their elements for all pairs $k_{i} \neq k_{j}$ in $\mathbf{k}(\mathbf{c})$, the intersection of the lattices $\boldsymbol{\Lambda}_{\mathbf{k}(\mathbf{c})}=\bigcap_{k \in \mathbf{k}(\mathbf{c})} \boldsymbol{\Lambda}_{k}$ is a 2D lattice, ${ }^{27,28}$ whose periodicity may be represented in terms of a basis matrix $\mathbf{V}_{\mathbf{k}(\mathbf{c})}$. The basis matrix $\mathbf{V}_{\mathbf{k}(\mathbf{c})}$ can be obtained using a least common right multiple computation for integer matrices (see Ref. 27 pp. 35-38). ${ }^{29}$

As it can be seen from the illustration in Fig. 3, a period of $\boldsymbol{\Lambda}_{\mathbf{k}(\mathbf{c})}$ includes at least one period of each of the constituent lattices. The overlay $h_{\mathbf{k}(\mathbf{c})}\left(\mathbf{x} ; \mathbf{d}_{\mathbf{k}(\mathbf{c})}\right)$ of the constituent halftones is then periodic over this lattice and thus the spatial average is obtained as

$\beta_{\mathbf{k}(\mathbf{c})}=\frac{1}{\left|\mathcal{U}_{\mathbf{k}(\mathbf{c})}\right|} \int_{\mathbf{x} \in \mathcal{U}_{\mathbf{k}(\mathbf{c})}} h_{\mathbf{k}(\mathbf{c})}(\mathbf{x}) d \mathbf{x}$,

where $\mathcal{U}_{\mathbf{k}(\mathbf{c})}$ denotes a unit cell of $\boldsymbol{\Lambda}_{\mathbf{k}(\mathbf{c})}$ and $\left|\mathcal{U}_{\mathbf{k}(\mathbf{c})}\right|$ the area of this unit cell. We note that for digital halftone screens, that is, screens defined on a common discrete periodic grid for any $k, l \in\{1, \ldots, K\}, \mathbf{V}_{k}^{-1} \mathbf{V}_{l}$ has only rational entries and thus the above assumption holds for any colorant combination in c. Situations in which the assumption does not hold may be viewed as a limiting case for out analysis, where $\left|\mathcal{U}_{\mathbf{k}(\mathbf{c})}\right| \rightarrow \infty$. Our ensuing frequency domain analysis [particularly Eq. (17)] still holds for these situations even though the basis matrix $\mathbf{V}_{\mathbf{k}(\mathbf{c})}$ does not exist.

From the Fourier transform properties it follows that 
$\beta_{\mathbf{k}(\mathbf{c})}=H_{\mathbf{k}(\mathbf{c})}(\mathbf{0})$,

where $H_{\mathbf{k}(\mathbf{c})}(\mathbf{u})$ represents the Fourier transform of $h_{\mathbf{k}(\mathbf{c})}(\mathbf{x})$ and $\mathbf{u}=[u, v]^{T}$ denotes the coordinates in frequency space. In other words, $\beta_{\mathbf{k}(\mathbf{c})}$ is the DC term of the frequency spectrum of the overlay of its constituent colorants. The Fourier transform of Eq. (9) yields

$H_{\mathbf{k}(\mathbf{c})}(\mathbf{u})=H_{k_{1}}^{\left(\mathbf{d}_{k_{1}}\right)}(\mathbf{u}) * \ldots * H_{k_{m}}^{\left(\mathbf{d}_{k_{m}}\right)}(\mathbf{u})$,

where $H_{k}^{\left(\mathbf{d}_{k}\right)}(\mathbf{u})$ represents the Fourier transform of the halftone image $h_{k}^{\left(\mathbf{d}_{k}\right)}(\mathbf{x})$. Let $S_{k}(\mathbf{u})$ represent the Fourier transform of the halftone spot function $s_{k}(\mathbf{x})$. Applying the shift and convolution property of the Fourier transform on Eq. (3), $H_{k}^{\left(\mathbf{d}_{k}\right)}(\mathbf{u})$ can be written as

$H_{k}^{\left(\mathbf{d}_{k}\right)}(\mathbf{u})=\frac{1}{\left|\mathbf{V}_{k}\right|} S_{k}(\mathbf{u}) \exp \left(-2 \pi j \mathbf{d}_{k}^{T} \mathbf{u}\right) \sum_{\mathbf{n}_{k}} \delta\left(\mathbf{u}-\mathbf{W}_{k} \mathbf{n}_{k}\right)$,

where the Fourier transform of the "comb" function $\Sigma_{\mathbf{n}_{k}} \delta\left(\mathbf{x}-\mathbf{V}_{k} \mathbf{n}_{k}\right)$ takes nonzero values on the elements of the reciprocal lattice of $\boldsymbol{\Lambda}_{k}$ (see Ref. 22, pp. 23-24), ${ }^{28}$ which is represented by

$\Lambda_{k}^{*}=\left\{\mathbf{W}_{k} \mathbf{n}_{k}=\left(\mathbf{V}_{k}^{-1}\right)^{T} \mathbf{n}_{k} \mid \mathbf{n}_{k} \in \mathbb{Z}^{2}\right\}$

where $\mathbf{W}_{k}=\left(\mathbf{V}_{k}^{-1}\right)^{T}$ represents the basis matrix for the reciprocal lattice $\boldsymbol{\Lambda}_{k}^{*}$.

Using these results we see that (see Appendix A)

$$
\begin{aligned}
H_{\mathbf{k}(\mathbf{c})}(\mathbf{u})= & \sum_{\mathbf{n}_{k_{1}}} \ldots \sum_{\mathbf{n}_{k_{m}}} \prod_{k \in \mathbf{k}(\mathbf{c})}\left(\frac{1}{\left|\mathbf{V}_{k}\right|} S_{k}\left(\mathbf{W}_{k} \mathbf{n}_{k}\right)\right. \\
& \left.\times \exp \left(-2 \pi j \mathbf{d}_{k}^{T} \mathbf{W}_{k} \mathbf{n}_{k}\right)\right) \delta\left(\mathbf{u}-\sum_{l \in \mathbf{k}(\mathbf{c})} \mathbf{W}_{l} \mathbf{n}_{l}\right),
\end{aligned}
$$

which can only take nonzero values if $\mathbf{u}=\Sigma_{k \in \mathbf{k}(\mathbf{c})} \mathbf{W}_{k} \mathbf{n}_{k}$. Let $\mathcal{N}_{\mathbf{k}(\mathbf{c})}$ represent the set $\left\{\left(\mathbf{n}_{k_{1}}, \ldots, \mathbf{n}_{k_{m}}\right) \mid \sum_{i=1}^{m} \mathbf{W}_{k_{i}} \mathbf{n}_{k_{i}}=\mathbf{0}\right\}$, which includes the indices of all the frequency vectors that sum up to the zero vector.

Then, Eq. (11) can be computed as

$$
\begin{aligned}
\beta_{\mathbf{k}(\mathbf{c})} & =H_{\mathbf{k}(\mathbf{c})}(\mathbf{0}) \\
& =\sum_{\left(\mathbf{n}_{k_{1}}, \ldots, \mathbf{n}_{k_{m}}\right) \in \mathcal{N}_{\mathbf{k}(\mathbf{c})}} \prod_{k \in \mathbf{k}(\mathbf{c})} \frac{1}{\left|\mathbf{V}_{k}\right|} S_{k}\left(\mathbf{W}_{k} \mathbf{n}_{k}\right) \exp \left(-2 \pi j \mathbf{d}_{k}^{T} \mathbf{W}_{k} \mathbf{n}_{k}\right) \\
& =\prod_{k \in \mathbf{k}(\mathbf{c})} \frac{1}{\left|\mathbf{V}_{k}\right|} S_{k}(\mathbf{0})+\sum_{\left(\mathbf{n}_{k_{1}}, \ldots, \mathbf{n}_{k_{m}}\right) \in \mathcal{N}_{\mathbf{k}(\mathbf{c})} \backslash\{\mathbf{0}\}} \prod_{k \in \mathbf{k}(\mathbf{c})} \frac{1}{\left|\mathbf{V}_{k}\right|} S_{k}\left(\mathbf{W}_{k} \mathbf{n}_{k}\right) \exp \left(-2 \pi j \mathbf{d}_{k}^{T} \mathbf{W}_{k} \mathbf{n}_{k}\right) \\
& =\prod_{k \in \mathbf{k}(\mathbf{c})} \beta_{k}+\sum_{\left(\mathbf{n}_{k_{1}}, \ldots, \mathbf{n}_{k_{m}}\right) \in \mathcal{N}_{\mathbf{k}(\mathbf{c})} \backslash\{\mathbf{0}\}} \prod_{k \in \mathbf{k}(\mathbf{c})} \frac{1}{\left|\mathbf{V}_{k}\right|} S_{k}\left(\mathbf{W}_{k} \mathbf{n}_{k}\right) \exp \left(-2 \pi j \mathbf{d}_{k}^{T} \mathbf{W}_{k} \mathbf{n}_{k}\right),
\end{aligned}
$$

where $\mathcal{N}_{\mathbf{k}(\mathbf{c})} \backslash\{\boldsymbol{0}\}$ denotes the elements in $\mathcal{N}_{\mathbf{k}(\mathbf{c})}$ with the exclusion of the all-zero vector $\mathbf{0}$. If $\mathcal{N}_{\mathbf{k}(\mathbf{c})} \backslash\{\mathbf{0}\}$ is a nonempty set, then the overlay of the halftones is said to be singular. ${ }^{16}$ Note that only terms indexed by variables of the summation symbol in Eq. (17) depend on the interseparation misregistration amounts.

\section{Conditions for Color Misregistration Insensitivity}

Denote by $R_{a v g}^{(\mathbf{0})}(\lambda)$ and $R_{a v g}^{(\mathbf{d})}(\lambda)$, the average spectra of "prints" with interseparation displacements 0 (perfect registration) and $\mathbf{d}=\left[\mathbf{d}_{1}, \ldots, \mathbf{d}_{K}\right]$ (misregistered), respectively. A difference in these terms represents a misregistrationinduced color shift [Strictly speaking, this is a shift in the average spectrum that will typically produce a corresponding color shift]. In this section, we consider the conditions under which these terms do not differ, yielding insensitivity to color misregistration in the average color. In Eq. (5), there are two elements that affect the value of these terms: spectral reflectances of the Neugebauer primaries, and fractional area coverages of the Neugebauer primaries. The former is affected by the spectral interactions of the colorants (inks) in their absorption bands of the spectra, the latter is a function of individual halftone separation periodicities, halftone spots, and the inter-separation misregistrations as shown in Eq. (17). These terms define conditions under which $R_{\text {avg }}(\lambda)$ is affected by interseparation misregistration as we show in the following subsections. We also observe here that the terms $\left\{\beta_{k}\right\}_{k=1}^{K}$, corresponding to the total fractional area covered by the individual colorants, are independent of misregistration.

\subsection{Spectral Sufficiency Condition}

If the colorants are transparent with nonoverlapping spectral absorption bands, it is well known that the resulting 


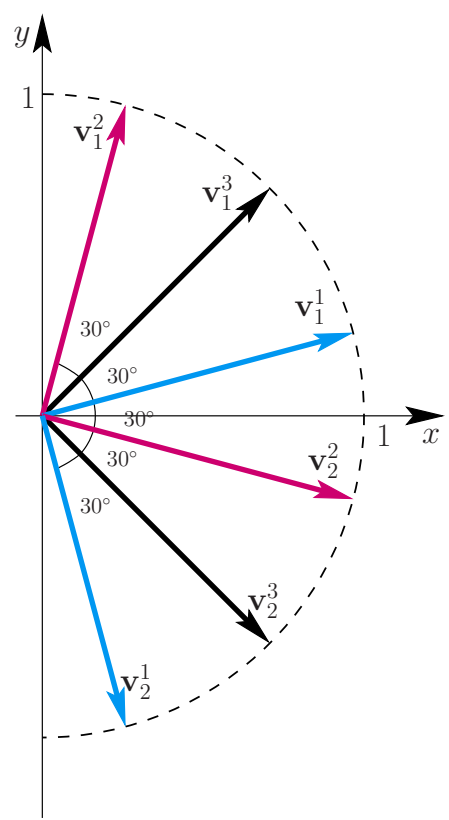

(a) Spatial domain

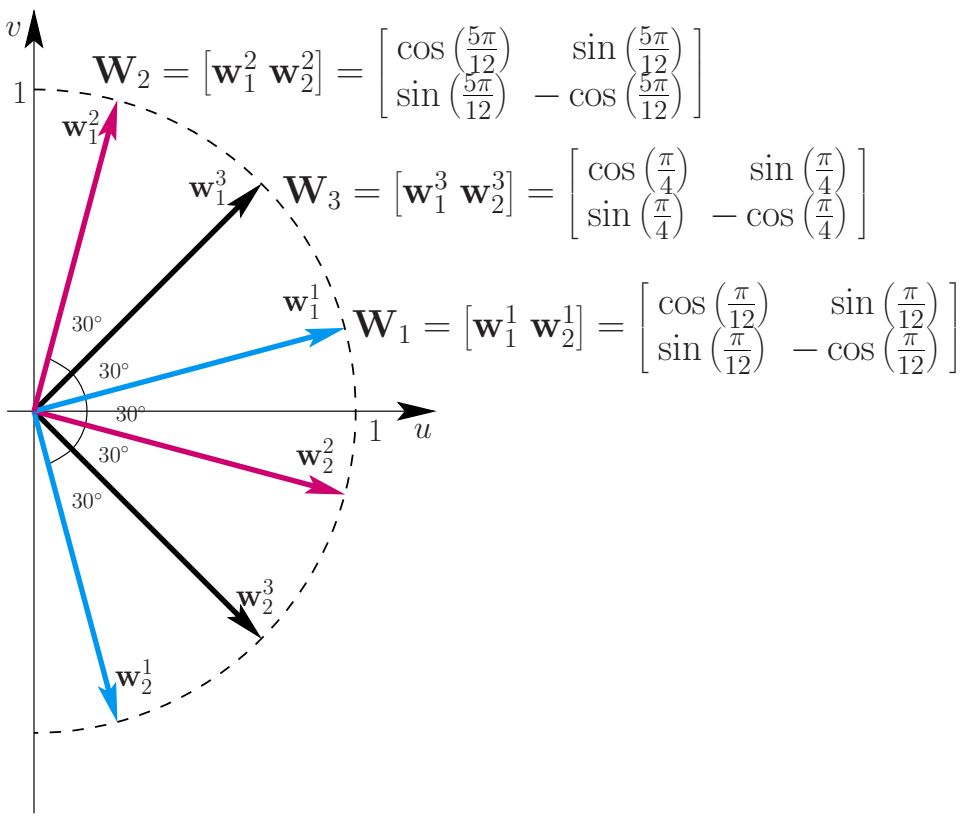

(b) Frequency domain

Fig. 4 Basis vectors for CMK colorants in conventional screens. (Color online only.)

color prints have no misregistration sensitivity. ${ }^{6}$ In our analysis, this can be seen by noting that in this scenario, one can represent the reflectance of a Neugebauer primary formed by the colorants in $\mathbf{k}(\mathbf{c})$ as $R_{\mathbf{k}(\mathbf{c})}(\lambda)$ $=R_{P}(\lambda) \Pi_{k \in \mathbf{k}(\mathbf{c})}\left[1-A_{k}(\lambda)\right]$, where $R_{P}(\lambda)$ is the reflectance of the paper substrate and $A_{k}(\lambda)$ is the absorptance of the $k$ 'th colorant. For a given wavelength $\lambda$, then there exists a single separation $k_{\lambda}$ for which the colorant has nonzero absorption at $\lambda$. Using this property, the average reflectance at $\lambda$ can be written as

$R(\lambda)=\left(\beta_{k_{\lambda}}\left[1-A_{k_{\lambda}}(\lambda)\right]^{1 / \gamma}+\left(1-\beta_{k_{\lambda}}\right)\right)^{\gamma} R_{P}(\lambda)$.

Because the $\beta_{k}$ terms corresponding to individual colorant separation fractional area coverages are insensitive to misregistration, $R(\lambda)$ is not affected by the interseparation misregistration. Thus, a color halftone is insensitive to interseparation misregistration if the aforementioned condition is satisfied. However, in most color printing systems, the colorants do not obey this condition [for instance, any pair of colorants that include the black $(K)$ colorant violates this requirement].

\subsection{Periodicity Sufficiency Condition}

Consider the expression in Eq. (17) in Sec. 3.3 for the fractional area of the colorant combination $\mathbf{k}(\mathbf{c})$. If $\mathcal{N}_{\mathbf{k}(\mathbf{c})} \backslash\{\boldsymbol{0}\}$ is an empty set, or in other words, none of the fundamental frequency vectors or their harmonics sum up to the zero vector, then the corresponding fractional area $\beta_{\mathbf{k}(\mathbf{c})}$ does not depend on the displacements $\left\{\mathbf{d}_{k}\right\}_{k \in \mathbf{k}(\mathbf{c})}$. If this property $\left(\mathcal{N}_{\mathbf{k}(\mathbf{c})} \backslash\{\boldsymbol{0}\}\right.$ empty) holds for every possible colorant combination, then the equivalence between the Neugebauer primary areas and areas of colorant combinations ensures that the Neugebauer primary areas $a_{c_{1} \ldots c_{K}}$ do not depend on the displacements $\left\{\mathbf{d}_{k}\right\}_{k=1}^{K}$. Therefore, in this scenario, the average spectrum of the printed halftone $R_{\text {avg }}(\lambda)$ is invariant to interseparation misregistration. A set of halftone screens for which the above property $\left[\mathcal{N}_{\mathbf{k}(\mathbf{c})} \backslash\{\boldsymbol{0}\}\right.$ empty for all $\mathbf{k}(\mathbf{c})]$ holds is referred to as a nonsingular halftone configuration. ${ }^{16}$

Let us visualize this case by examining the conventional 30-deg angular separation equifrequency CMK halftone screen overlay, which is commonly used in lithographic printing systems. Let $\mathbf{V}_{1}, \mathbf{V}_{2}$, and $\mathbf{V}_{3}$, which are formed by the basis vectors shown in Fig. 4(a), represent the basis matrices for the lattices $\boldsymbol{\Lambda}_{1}, \boldsymbol{\Lambda}_{2}$, and $\boldsymbol{\Lambda}_{3}$ for $\mathrm{C}, \mathrm{M}$, and $\mathrm{K}$ separations, respectively. We first consider the superposition of any two of these separations-for example, $\mathrm{C}$ and M. To compute $\beta_{12}$, we first consider the set of indices $\mathcal{N}_{12} \backslash\{\boldsymbol{0}\}$ in Eq. (17). From the frequency domain basis matrices shown in Fig. 4(b), it can be seen that there do not exist any $\left(\mathbf{n}_{1}, \mathbf{n}_{2}\right) \in \mathbb{Z}^{2}$ that can satisfy $\mathbf{W}_{1} \mathbf{n}_{1}+\mathbf{W}_{2} \mathbf{n}_{2}=\mathbf{0}$. Thus, $\mathcal{N}_{12} \backslash\{\boldsymbol{0}\}$ is an empty set and $\beta_{12}$ is invariant to interseparation misregistration. Similarly, it can be shown that $\mathcal{N}_{13} \backslash\{\boldsymbol{0}\}$ and $\mathcal{N}_{23} \backslash\{\boldsymbol{0}\}$ are also empty sets and consequently $\beta_{13}$ and $\beta_{23}$ are also invariant to interseparation misregistration. Note that, in these cases, $\beta_{\mathbf{k}(\mathbf{c})}$ terms are only determined by the first term in Eq. (17), which is the multiplication of the DC terms in each individual separation that constitutes $\mathbf{k}(\mathbf{c})$ and, therefore, the statistical randomness condition assumed by Demichel equations is satisfied.

Now consider overlay of all three of the separations and the $\beta_{123}$. In this case, we can see that $\mathcal{N}_{123} \backslash\{\boldsymbol{0}\}$ is not an empty set and the overlay is singular. For example, $\mathbf{n}_{1}$ $=[-1,0]^{T}, \mathbf{n}_{2}=[1,0]^{T}$, and $\mathbf{n}_{3}=[0,1]^{T}$ is a member of $\mathcal{N}_{123} \backslash\{\mathbf{0}\}$. The set $\mathcal{N}_{123} \backslash\{\mathbf{0}\}$ has an infinite number of elements and color sensitivity to misregistration is expected 


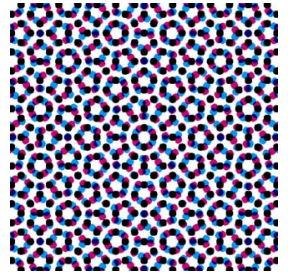

Clear-centered rosette

(a)

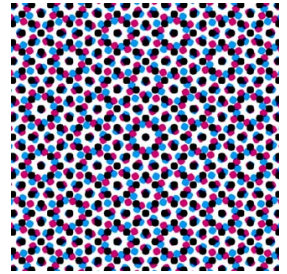

Dot-centered rosette

(b)
Fig. 5 Sensitivity of CMK overlap in conventional screens to misregistration. A half period displacement of each of the screen transforms the microstructure from the clear-centered rosette of (a) to the dot-centered rosette in (b). (Color online only.)

for the overlay of these three separations. Figure 5 illustrates this behavior of the conventional CMK halftone overlay. The configuration of Fig. 5(a), known as the clearcentered rosette, transforms into the so-called dot-centered rosette configuration of Fig. 5(b) when each of the separations is displaced by half its lattice period. The change from Fig. 5(a) and 5(b) illustrates the substantial change in the microstructure due to interseparation misregistration in a singular overlay.

\subsection{Spot Function Dependence}

From Eq. (17), we observe that if the set $\mathcal{N}_{\mathbf{k}(\mathbf{c})} \backslash\{\mathbf{0}\}$ is nonempty, this alone does not ensure that $R_{a v g}^{(\mathbf{d})}(\lambda)$ and $R_{a v g}^{(\mathbf{0})}(\lambda)$ differ, because $\beta_{\mathbf{k}(\mathbf{c})}$ terms are functions of the constituent halftone spots and the interseparation misregistration amounts. Depending on these, interseparation misregistration may still have no effect on the average color of the halftone if the summation in Eq. (17) is either zero or remains constant as the displacement $\mathbf{d}$ is varied. This condition holds trivially when colorant coverages take on only values of 0 or $100 \%$, but it can also hold for nontrivial cases, as we illustrate next by means of an example.

Consider an overlay of two separations with lattices $\Lambda_{1}$ and $\boldsymbol{\Lambda}_{2}$ having the basis matrices

$\mathbf{V}_{1}=\left[\begin{array}{cc}M & 0 \\ 0 & M\end{array}\right]$ and $\quad \mathbf{V}_{2}=\left[\begin{array}{cc}M & M \\ M & -M\end{array}\right]$,

respectively. Let us define the corresponding halftone spot functions $s_{1}(\mathbf{x})$ and $s_{2}(\mathbf{x})$ as shown in Fig. 6 within unit cells of the constituent lattices outlined by the dashed lines.

These functions can also be represented as

$s_{1}(\mathbf{x})=\operatorname{rect}\left(\frac{x-y}{M}\right) \operatorname{rect}\left(\frac{x+y}{M}\right)$

and

$s_{2}(\mathbf{x})=\operatorname{rect}\left(\frac{x}{M}\right) \operatorname{rect}\left(\frac{y}{M}\right)$.

The respective Fourier transforms of these functions can be written as

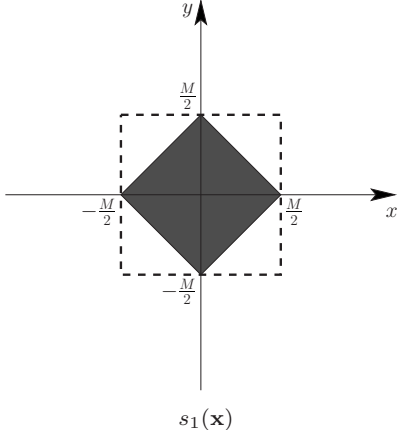

(a)

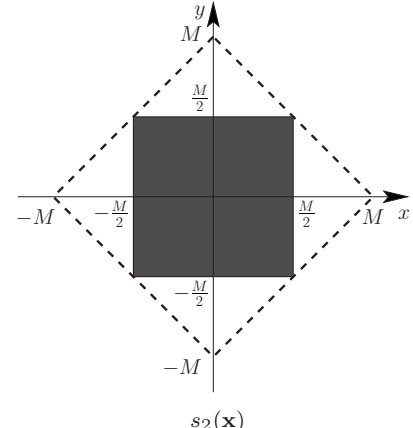

(b)
Fig. 6 Spot functions $s_{1}(\mathbf{x})$ and $s_{2}(\mathbf{x})$ exhibit misregistration invariance despite a potentially sensitive geometry.

$S_{1}(\mathbf{u})=\frac{M^{2}}{2} \operatorname{sinc}\left(\frac{u-v}{2} M\right) \operatorname{sinc}\left(\frac{u+v}{2} M\right)$

and

$S_{2}(\mathbf{u})=M^{2} \operatorname{sinc}(u M) \operatorname{sinc}(v M)$.

The summation in Eq. (17) requires the value of the previous functions at the frequency coordinates $\mathbf{W}_{1} \mathbf{n}_{1}$ and $\mathbf{W}_{2} \mathbf{n}_{2}$

$$
\begin{aligned}
S_{1}\left(\mathbf{W}_{1} \mathbf{n}_{1}\right) & =S_{1}\left(\frac{1}{M}\left[\begin{array}{l}
n_{1 x} \\
n_{1 y}
\end{array}\right]\right) \\
& =\frac{M^{2}}{2} \operatorname{sinc}\left(\frac{n_{1 x}-n_{1 y}}{2}\right) \operatorname{sinc}\left(\frac{n_{1 x}+n_{1 y}}{2}\right)
\end{aligned}
$$

and

$$
\begin{aligned}
S_{2}\left(\mathbf{W}_{2} \mathbf{n}_{2}\right) & =S_{2}\left(\frac{1}{2 M}\left[\begin{array}{l}
n_{2 x}+n_{2 y} \\
n_{2 x}-n_{2 y}
\end{array}\right]\right) \\
& =M^{2} \operatorname{sinc}\left(\frac{n_{2 x}+n_{2 y}}{2}\right) \operatorname{sinc}\left(\frac{n_{2 x}-n_{2 y}}{2}\right) .
\end{aligned}
$$

The indices $\mathbf{n}_{1}$ and $\mathbf{n}_{2}$ for the summation in Eq. (17) are defined by the condition

$$
\mathbf{W}_{1} \mathbf{n}_{1}+\mathbf{W}_{2} \mathbf{n}_{2}=\frac{1}{M}\left[\begin{array}{c}
n_{1 x}+\frac{n_{2 x}+n_{2 y}}{2} \\
n_{1 y}+\frac{n_{2 x}-n_{2 y}}{2}
\end{array}\right]=\mathbf{0} .
$$

The previous relation implies that only indices such that $n_{2 x}$ or $n_{2 y}$ are both odd or both even can contribute terms in $\mathcal{N}_{12} \backslash\{\boldsymbol{0}\}$. However, in these cases, the value of the sinc functions in Eq. (24) is zero. As Eq. (17) suggests, this ensures $\beta_{12}$ is the multiplication of the fractional area coverages of the individual separations, and therefore, the characteristics of the halftone spots can define a condition to ensure that $R_{\text {avg }}(\lambda)$ is insensitive to interseparation misregistration. In Fig. 7, we show an example using this configuration in which colorant overlap area does not change even though the microstructure changes due to interseparation misregistration. We note that for this specific configuration, the invariance under misregistration depends only on the 


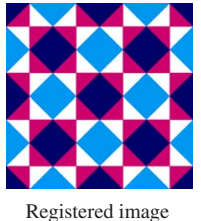

(a)

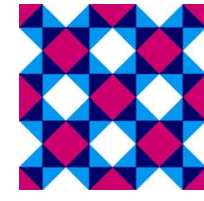

Misregistered image 1

(b)

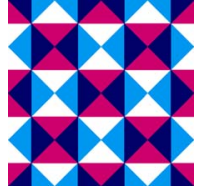

(c)

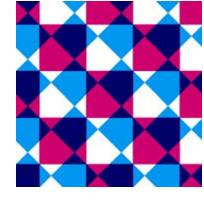

(d)
Fig. 7 Halftone images generated by using the periodicities and spot functions shown in Figs. 6(a) and 6(b) for $\mathrm{C}$ and $\mathrm{M}$ colorants, respectively. Observe that Neugebauer primary areas do not change for this configuration with change in misregistration displacement. (Color online only.)

spot function $s_{2}(\mathbf{x})$ and holds for any arbitrary choice of the spot function $s_{1}(\mathbf{x})$.

\subsection{Invariant Misregistrations}

Now we consider the invariant misregistration combinations, that is, the separation displacements for which we are assured zero color shift with respect to the perfectly registered print. It is readily seen from Eq. (17) that if the displacement of the $k^{\prime}$ th separation is a point on the corresponding lattice, the term $\mathbf{d}_{k}^{T} \mathbf{W}_{k} \mathbf{n}_{k}$ is integer-valued and the result of the summation is identical to that for a perfectly registered halftone. This represents the trivial case when the interseparation displacements are matched to the separations' lattice periodicities. Invariance is, however, also achievable for nontrivial displacements, as we illustrate next. We assume in our analysis that for the lattices under consideration, the basis matrices satisfy the constraint $\mathbf{V}_{k_{i}}^{-1} \mathbf{V}_{k_{j}} \in \mathbb{Q}^{2 \times 2}$, where $Q$ is the set of rational numbers so that the intersection and sum lattices are defined for any subset of lattices. ${ }^{27,28}$

Consider, again, an overlay of two separations with lattices $\boldsymbol{\Lambda}_{1}$ and $\boldsymbol{\Lambda}_{2}$. In this case, Eq. (17) simplifies to

$$
\begin{aligned}
\beta_{12}= & \beta_{1} \beta_{2}+\sum_{\left(\mathbf{n}_{1}, \mathbf{n}_{2}\right) \in \mathcal{N}_{12} \backslash\{0\}} \frac{1}{\left|\mathbf{V}_{1}\right|\left|\mathbf{V}_{2}\right|} S_{1}\left(\mathbf{W}_{1} \mathbf{n}_{1}\right) S_{2}\left(\mathbf{W}_{2} \mathbf{n}_{2}\right) \\
& \times \exp \left[-2 \pi j\left(\mathbf{d}_{1}^{T} \mathbf{W}_{1} \mathbf{n}_{1}+\mathbf{d}_{2}^{T} \mathbf{W}_{2} \mathbf{n}_{2}\right)\right] .
\end{aligned}
$$

If the terms $\exp \left[-2 \pi j\left(\mathbf{d}_{1}^{T} \mathbf{W}_{1} \mathbf{n}_{1}+\mathbf{d}_{2}^{T} \mathbf{W}_{2} \mathbf{n}_{2}\right)\right]$ in the above summation are unity for all $\left(\mathbf{n}_{1}, \mathbf{n}_{2}\right) \in \mathcal{N}_{12} \backslash\{\boldsymbol{0}\}$, then we can readily see that the value of $\beta_{12}$ is equal to the value obtained for the perfectly registered case $\left(\mathbf{d}_{1}=\mathbf{d}_{2}=\mathbf{0}\right)$. We readily see that this happens if the terms $\left(\mathbf{d}_{1}^{T} \mathbf{W}_{1} \mathbf{n}_{1}+\mathbf{d}_{2}^{T} \mathbf{W}_{2} \mathbf{n}_{2}\right)$ are integer-valued for all $\left(\mathbf{n}_{1}, \mathbf{n}_{2}\right)$ $\in \mathcal{N}_{12} \backslash\{\boldsymbol{0}\}$. Suppose $\left(\mathbf{n}_{1}, \mathbf{n}_{2}\right) \in \mathcal{N}_{12} \backslash\{\mathbf{0}\}$, that is, they are nonzero integer vectors satisfying $\mathbf{W}_{1} \mathbf{n}_{1}+\mathbf{W}_{2} \mathbf{n}_{2}=\mathbf{0}$. Then, $\quad \mathbf{W}_{2} \mathbf{n}_{2}=-\mathbf{W}_{1} \mathbf{n}_{1} \quad$ and $\quad\left(\mathbf{d}_{1}^{T} \mathbf{W}_{1} \mathbf{n}_{1}+\mathbf{d}_{2}^{T} \mathbf{W}_{2} \mathbf{n}_{2}\right)$ $=\left(\mathbf{d}_{1}-\mathbf{d}_{2}\right)^{T} \mathbf{W}_{1} \mathbf{n}_{1}=\left(\mathbf{d}_{2}-\mathbf{d}_{1}\right)^{T} \mathbf{W}_{2} \mathbf{n}_{2}$. Now $\mathbf{W}_{2} \mathbf{n}_{2} \in \boldsymbol{\Lambda}_{2}^{*}$ and $-\mathbf{W}_{1} \mathbf{n}_{1} \in \Lambda_{1}^{*}$, hence $\mathbf{w}=\mathbf{W}_{2} \mathbf{n}_{2}=-\mathbf{W}_{1} \mathbf{n}_{1} \in \Lambda_{1}^{*} \cap \Lambda_{2}^{*}$ and vice versa if $\mathbf{w} \in \boldsymbol{\Lambda}_{1}^{*} \cap \boldsymbol{\Lambda}_{2}^{*}$, then there exists a $\mathbf{w}=\mathbf{W}_{1} \mathbf{n}_{1}^{\prime}=$ $-\mathbf{W}_{2} \mathbf{n}_{2}^{\prime}$, whence $\left(\mathbf{n}_{1}^{\prime}, \mathbf{n}_{2}^{\prime}\right) \in \mathbb{Z}^{2}$ such that $\mathbf{W}_{1} \mathbf{n}_{1}^{\prime}+\mathbf{W}_{2} \mathbf{n}_{2}^{\prime}=\mathbf{0}$. From the definition of the reciprocal lattice, it follows that $\left(\mathbf{d}_{1}-\mathbf{d}_{2}\right)^{T} \mathbf{W}_{1} \mathbf{n}_{1}$ is an integer if and only if $\mathbf{d}_{2}-\mathbf{d}_{1}$ is an element of $\left(\boldsymbol{\Lambda}_{1}^{*} \cap \boldsymbol{\Lambda}_{2}^{*}\right)^{*}$, that is, the reciprocal lattice of $\Lambda_{1}^{*} \cap \Lambda_{2}^{*}$. Because $\left(\Lambda_{1}^{*} \cap \Lambda_{2}^{*}\right)^{*}=\Lambda_{1}+\Lambda_{2}, \mathbf{d}_{1}-\mathbf{d}_{2} \in \boldsymbol{\Lambda}_{1}+\boldsymbol{\Lambda}_{2}$ defines a sufficient condition that ensures $\beta_{12}$ is invariant to misregistration, where

$\Lambda_{1}+\Lambda_{2} \stackrel{\text { def }}{=}\left\{\mathbf{x}_{1}+\mathbf{x}_{2} \mid \mathbf{x}_{1} \in \Lambda_{1}, \mathbf{x}_{2} \in \Lambda_{2}\right\}$

is the sum lattice of $\boldsymbol{\Lambda}_{1}, \boldsymbol{\Lambda}_{2}$. One can also infer that the sum lattice $\boldsymbol{\Lambda}_{1}+\boldsymbol{\Lambda}_{2}$ defines the periodicity of color shift in the interseparation displacement. The color shift for any interseparation displacement $\mathbf{d}^{12}=\mathbf{d}_{1}-\mathbf{d}_{2}$ is equal to the color shift obtained with an equivalent displacement $\mathbf{d}_{e}^{12}$ lying in the unit cell of $\boldsymbol{\Lambda}_{1}+\boldsymbol{\Lambda}_{2}$. The equivalent displacement $\mathbf{d}_{e}^{12}$ is "d $\mathbf{d}^{12}$ modulo $\boldsymbol{\Lambda}_{1}+\boldsymbol{\Lambda}_{2}$," that is, the unique vector in the set $\left\{\mathbf{d}^{12}+\mathbf{V}_{\boldsymbol{\Lambda}_{1}+\boldsymbol{\Lambda}_{2}} \mathbf{n} \mid \mathbf{n} \in \mathbb{Z}^{2}\right\}$ that lies in the unit cell of the sum

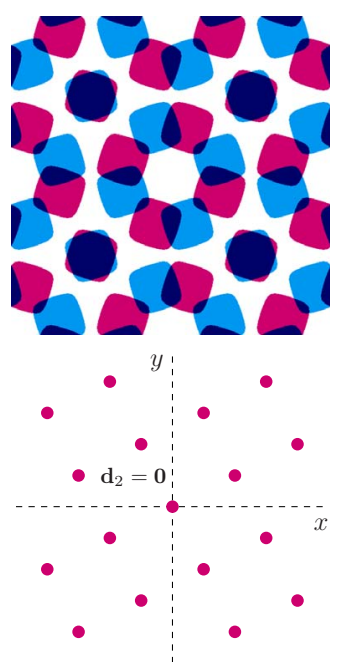

(a)

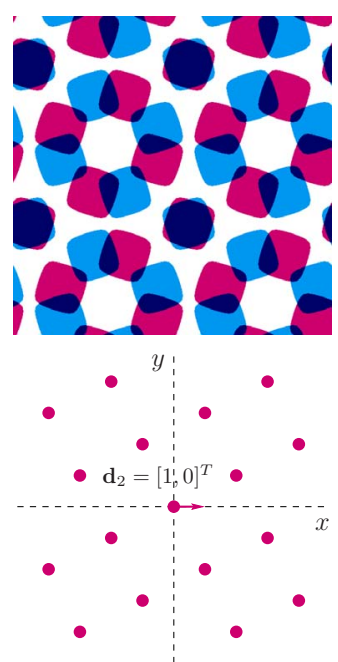

(b)

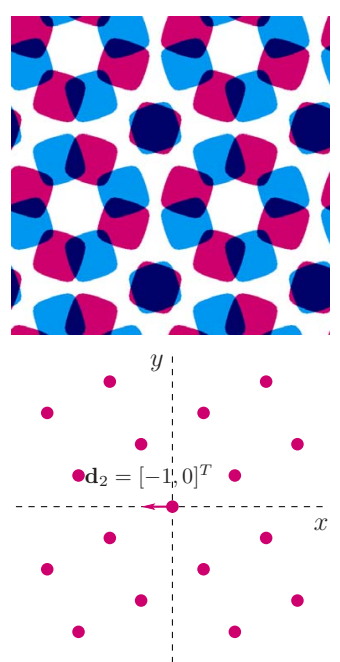

(c)

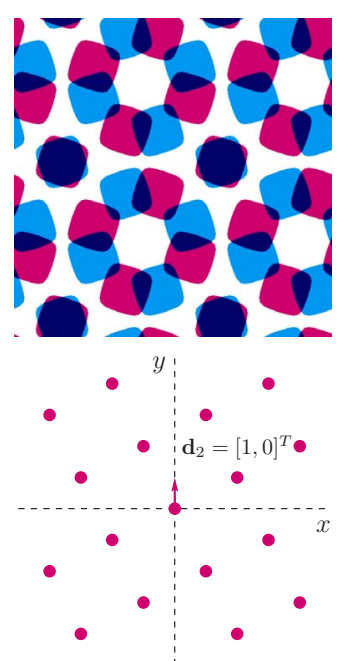

(d)

Fig. 8 Invariant misregistrations. Panel (a) shows perfect registration, and (b), (c), and (d) show nontrivial misregistrations under which average color is invariant. The top half of each subfigure shows an integral number of periods of the intersection lattice and the lower half shows the displacement misregistration vector $\mathbf{d}_{2}$ on the lattice $\Lambda_{2}$. Note that $\mathbf{d}_{1}=\mathbf{0}$ and $\mathbf{d}_{2} \in \boldsymbol{\Lambda}_{1}+\boldsymbol{\Lambda}_{2}$ for all configurations and Neugebauer primary areas are invariant to misregistration in these cases. (Color online only.) 
lattice $\boldsymbol{\Lambda}_{1}+\boldsymbol{\Lambda}_{2}$, where $\mathbf{V}_{\Lambda_{1}+\boldsymbol{\Lambda}_{2}}$ denotes the basis matrix for $\boldsymbol{\Lambda}_{1}+\boldsymbol{\Lambda}_{2}$. A basis matrix for $\boldsymbol{\Lambda}_{1}+\boldsymbol{\Lambda}_{2}$ can be computed as the greatest common left divisor (gcld) of the basis matrices for $\boldsymbol{\Lambda}_{1}$ and $\boldsymbol{\Lambda}_{2}$.

In the general $K$-separation scenario, as shown in Appendix $\mathrm{B}$, the invariant misregistrations are characterized as constraints on the pairwise displacements expressed mathematically as

$\mathbf{d}_{i}-\mathbf{d}_{j} \in \Lambda_{i}+\Lambda_{j}$ for all pairs $i \neq j$ in $\{1, \ldots, K\}$.

In addition, one can infer this also defines the periodicity of the misregistration-induced color shift in the space of interseparation displacement vectors $\mathbf{d}_{i}-\mathbf{d}_{j}$, for all $i, j$ $\in\{1, \ldots, K\}$. Thus, if $\left(\mathbf{d}_{1}, \ldots, \mathbf{d}_{K}\right)$ and $\left(\mathbf{d}_{1}^{\prime}, \ldots, \mathbf{d}_{K}^{\prime}\right)$ are two misregistration displacement vectors, their misregistrationinduced color shift is equal if for all pairs of separations $i$ and $j,\left(\mathbf{d}_{i}-\mathbf{d}_{j}\right)$ is congruent with $\left(\mathbf{d}_{i}^{\prime}-\mathbf{d}_{j}^{\prime}\right)$ modulo the sum lattice $\boldsymbol{\Lambda}_{i}+\boldsymbol{\Lambda}_{j}$, that is, $\left(\mathbf{d}_{i}-\mathbf{d}_{j}\right)-\left(\mathbf{d}_{i}^{\prime}-\mathbf{d}_{j}^{\prime}\right) \in \boldsymbol{\Lambda}_{i}+\boldsymbol{\Lambda}_{j}$. In Fig. 8 , we show color misregistration invariant configuration examples generated using the halftone periodicities shown in Fig. 3.

\section{Quantitative Evaluation of Misregistration- Induced Color Shifts}

The analysis of the preceding section characterized the conditions under which misregistration sensitivity may be encountered without quantifying the amount of color shift (i.e., sensitivity). In this section, we consider a simulation model that builds upon the analysis already presented and allows quantitative evaluation of color misregistration sensitivity. For this purpose, we compute the average reflectance using our model of Eq. (5) for two cases: one corresponding to perfectly registered separations and the other for the shift in consideration. These values may then be transformed to the approximately perceptually uniform Commission Internationale d' Eclairage L*a*b (CIELAB) color space. ${ }^{30}$ The color difference resulting from the misregistration can then be obtained in $\Delta E_{a b}^{*}$ units as the Euclidean distance between the CIELAB pairs. We are interested in the dependence of this color difference on the halftone lattices $\left\{\boldsymbol{\Lambda}_{k}\right\}_{k=1}^{K}$, the colorant area coverages $\left\{\beta_{k}\right\}_{k=1}^{K}$, and the separation displacements $\left\{\mathbf{d}_{k}\right\}_{k=1}^{K}$. The overall system for quantitative estimation of the color shift is illustrated in Fig. 9. By repeating the process for different values of these elements as well as Neugebauer primary spectra, and YN parameter $\gamma$, we can obtain quantitative estimates of the misregistration-induced color shift as a function of these parameters. The process requires a calculation of the colorant overlap areas $\beta_{\mathbf{k}(\mathbf{c})}$, which we consider next.

\subsection{Spatial Domain Computation of $\beta_{\mathbf{k}(\mathbf{c})}$}

In Sec. 3.3, in the analysis culminating in Eq. (16), we illustrated how the fractional Neugebauer primary areas can be computed in the frequency domain. Equivalent spatial domain expressions that are more suitable for simulations can be obtained through algebraic manipulation (see Appendix D)

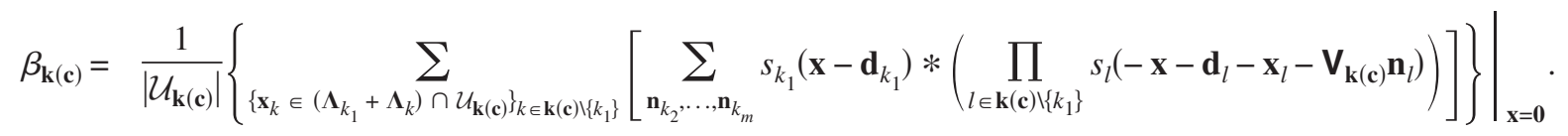

The first summation in the previous equation may be interpreted as adding up overlap areas of a reference spot of separation $k_{1}$ with neighboring spots of all remaining separations $k_{2}, \ldots, k_{m}$. The $\mathbf{x}_{k}$ 's in the first summation represents displacements of spots other than the $k_{1}$ 'th separation that potentially intersect with the spot $s_{k_{1}}\left(\mathbf{x}-\mathbf{d}_{k_{1}}\right)$ of the $k_{1}$ 'th separation. In Rogers's ${ }^{15}$ analysis for the overlay of twoscreens, the "dot neighbor distribution" corresponds to a "projection" of these displacements along the radial axis (for $K=2$ case)

\subsection{Halftone Spot Model}

The computation in Eq. (29) requires the halftone spot functions $\left\{s_{k}(\mathbf{x})\right\}_{k \in \mathbf{k}(\mathbf{c})}$ for the respective separations. In practice, these functions are produced as a result of thresholding the contone image for the $k$ 'th separation $\tau_{k}(\mathbf{x})$ against a threshold function for that separation $T_{k}(\mathbf{x})$. For most reasonable threshold functions, the spot function $s_{k}(\mathbf{x})$ may be uniquely determined by the corresponding colorant area coverage $\beta_{k}$. For orthogonal screens, a useful threshold function was defined by Pellar and Green ${ }^{31,32}$ as

$T(\mathbf{x})=\cos \left(2 \pi f_{x} x\right)+\cos \left(2 \pi f_{y} y\right)$,

where $f_{x}$ and $f_{y}$ are the screen frequencies, $x$ and $y$ are the respective spatial coordinates (along two orthogonal spatial directions) [In digital halftoning, this threshold function is usually defined as a discrete array on a discretized unit cell of the corresponding lattice, we will however find the analytic representation more convenient for our purposes]. For an arbitrary (possibly nonorthogonal) halftone, whose periodicity is given by the lattice $\boldsymbol{\Lambda}_{k}$, we generalize the equation to obtain the threshold function

$T_{k}(\mathbf{x})=\cos \left(2 \pi\left|\mathbf{w}_{1}^{k}\right| x^{\prime}\right)+\cos \left(2 \pi\left|\mathbf{w}_{2}^{k}\right| y^{\prime}\right)$,

where $\mathbf{W}_{k}=\left[\begin{array}{ll}\mathbf{w}_{1}^{k} & \mathbf{w}_{2}^{k}\end{array}\right]=\left[\begin{array}{cc}w_{x_{1}}^{k} & w_{x_{2}}^{k} \\ w_{y_{1}}^{k} & w_{y_{2}}^{k}\end{array}\right]$ is the basis matrix of $\boldsymbol{\Lambda}_{k}^{*}$.

The transformation 


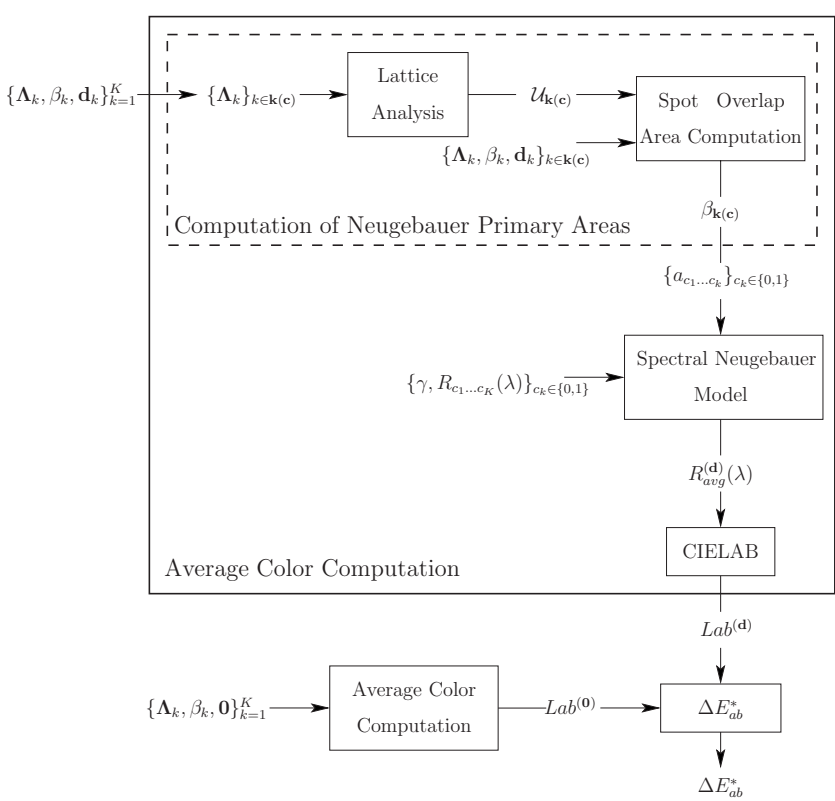

Fig. 9 Simulation model for quantitative estimation of color shift induced by interseparation misregistration for a clustered-dot color halftone.

$\left[\begin{array}{l}x^{\prime} \\ y^{\prime}\end{array}\right]=\left[\begin{array}{cc}\cos \left(\phi_{k}^{x}\right) & -\sin \left(\phi_{k}^{x}\right) \\ \sin \left(\phi_{k}^{y}\right) & \cos \left(\phi_{k}^{y}\right)\end{array}\right]\left[\begin{array}{l}x \\ y\end{array}\right]$

represents a shearing of the coordinate system that ensures a period of Eq. (31) maps to a unit cell of $\boldsymbol{\Lambda}_{k}$, and $\phi_{k}^{x}=\arctan \left(w_{y_{1}}^{k} / w_{x_{1}}^{k}\right)$ and $\phi_{k}^{y}=\left(w_{y_{2}}^{k} / w_{x_{2}}^{k}\right)$.

Individual colorant halftone separations may be obtained by thresholding the contone value for the colorant channel against the corresponding threshold function. Specifically,

$h_{k}(\mathbf{x})=\left\{\begin{array}{cc}1 & \text { if } \tau_{k}(\mathbf{x})>T_{k}(\mathbf{x}), \\ 0 & \text { otherwise, }\end{array}\right.$

defines the $k$ 'th halftone separation. For spatially constant contone images $\left[\tau_{k}(\mathbf{x})=\tau_{k}, \forall \mathbf{x}\right]$ the resulting halftone corresponds to the model of Eq. (2), however, the corresponding spot functions $\left\{s_{k}(\mathbf{x})\right\}_{k=1}^{K}$ do not allow for a closed form computation of the convolution terms in Eq. (29) and these must therefore be obtained by computer simulation.

For quantitative evaluation of the colorant overlap area $\beta_{\mathbf{k}(\mathbf{c})}$, we consider a finely sampled representation of the unit cell $\mathcal{U}_{\mathbf{k}(\mathbf{c})}$ and corresponding to each pixel of this sampled representation generate binary halftone separation values using Eq. (33). The area $\beta_{\mathbf{k}(\mathbf{c})}$ may then be obtained by counting the fraction of pixels at which the colorants overlap.
Accurate computations using this methodology require high resolution grids. In addition, in some configurations, the size of $\mathcal{U}_{\mathbf{k}(\mathbf{c})}$ may become unwieldy for the generation of images. We therefore consider a further simplification and in practice use either of these two approaches as appropriate.

\subsubsection{Simplified analytic halftone spot model}

The computation and storage requirements can be significantly reduced by using simple analytic spot functions that provide realistic approximations to actual halftone spot shapes for which overlap areas are obtained more readily from geometric relations. For this simplified model, we assume $\mathbf{w}_{1}^{k}$ and $\mathbf{w}_{2}^{k}$ are orthogonal to each other and $\left|\mathbf{w}_{1}^{k}\right|$ $=\left|\mathbf{w}_{2}^{k}\right|$ because orthogonal equifrequency halftone screens are commonly employed in clustered-dot color halftoning. Under this assumption, we examine the dependence of the spot function on the area coverage $\beta_{k}$ as shown in Fig. 10, where a linear contone ramp (ranging from white to black) has been thresholded using the function of Eq. (31). We observe that the gray levels progressing from highlight to midtone can be modeled as growing black spots on a white background up to $\beta=0.5$. From midtone to shadow gray levels, the halftone spots can be modeled as shrinking white holes on a black background, where the holes are displaced by half the lattice period relative to the black spots. Based on the observed shapes in Fig. 10, we model these spots and holes as circles in highlights and shadows, and squares in midtone gray levels. A closeness metric is employed to determine the fractional area coverage at which the model switches between the circle and square approximations. Based on the symmetry, it suffices to consider area coverages $\beta \in[0,0.5]$ to select the switch point. For a given $\beta$, let $s^{\beta}(\mathbf{x})$ denote the spot obtained from Eq. (33) with the corresponding area coverage. The error in approximating $s^{\beta}(\mathbf{x})$ by a circular or square spot is then evaluated as the area that lies in $s^{\beta}(\mathbf{x})$ or the approximating spot function, but not both (also called the symmetric difference or XOR). The shape (circle or square) providing the closest approximation is then used to approximate $s^{\beta}(\mathbf{x})$ as illustrated in Fig. 11(a). For the gray level used in the figure, we can see that the closer approximation for this specific case corresponds to the circle. Overlap area difference between $s^{\beta}(\mathbf{x})$ and the closest circle and square approximation is shown in Fig. 11(b) as a function of the area coverage $\beta$. As expected, in highlights and shadows, circular spots provide a closer approximation than square spots and vice versa in midtones. At approximately $\beta=0.35$, the error curves for square and circular spots intersect. Therefore, for $\beta \in[0.35,0.65]$, we use the square halftone spot approximation for $s^{\beta}(\mathbf{x})$, and for $\beta \in[0,0.35) \cup(0.65,1]$, we use the circular approximation.

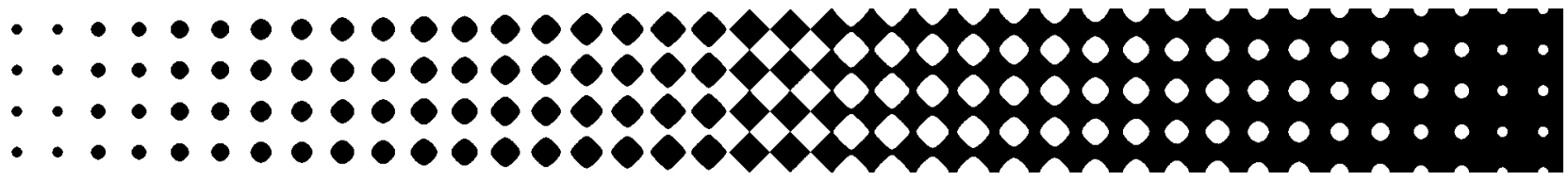

Fig. 10 Spot growth with the threshold function defined in Eq. (31) when $\left|\mathbf{w}_{1}\right|=\left|\mathbf{w}_{2}\right|$, and $\mathbf{w}_{1}$ and $\mathbf{w}_{2}$ are orthogonal. 

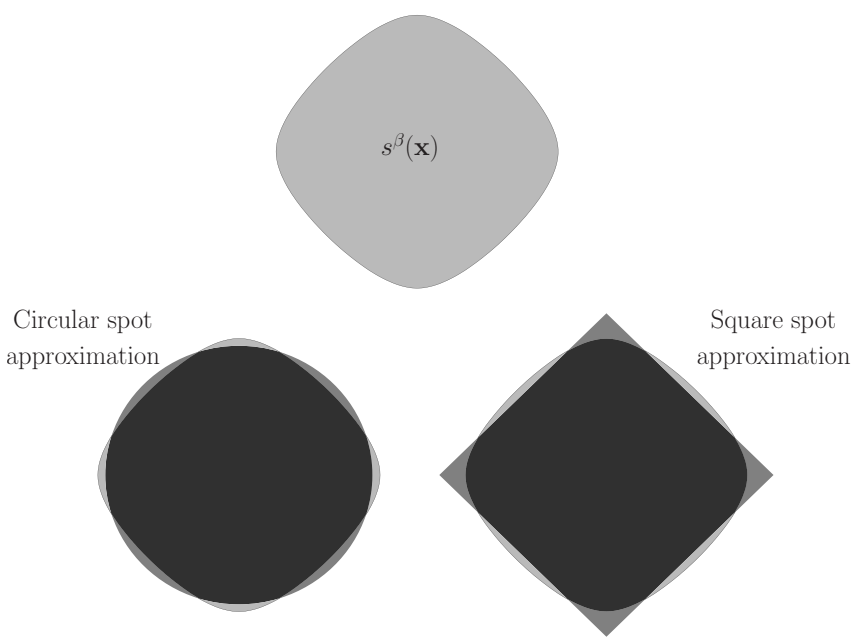

(a)

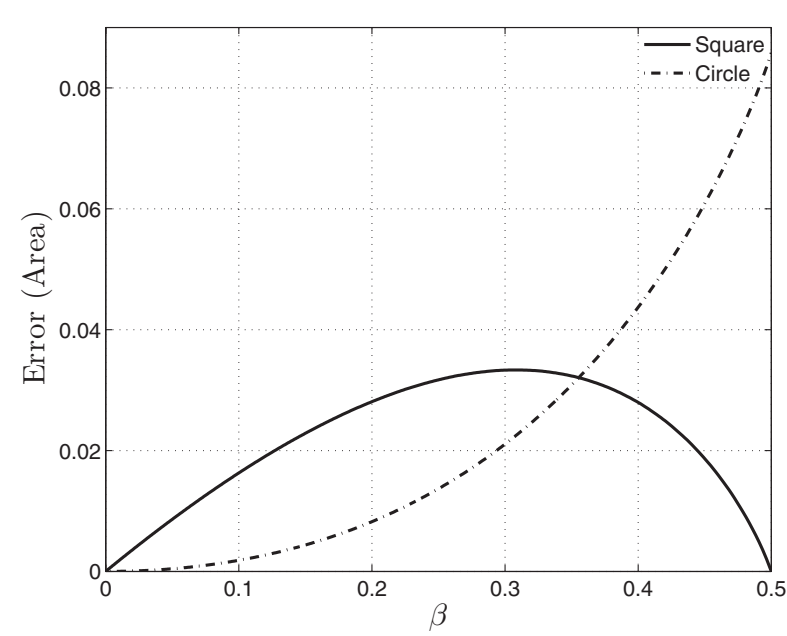

(b)

Fig. 11 Circle and square spot approximations to halftone spot function $s^{\beta}(\mathbf{x})$. Panel (a) shows an example of best approximations, (b) shows the error for the best circle and square approximations to $s^{\beta}(\mathbf{x})$ as a function of fractional area coverage $\beta$, where the error corresponds to the area lying in $s^{\beta}(\mathbf{x})$ or the approximation, but not both.

Assuming the halftone spot of a separation is either a circle or a square, a composite halftone image is composed of the overlay of these shapes at different displacements. The intersection of circles and squares can be found using simple algebraic methods, ${ }^{15,33}$ and once these points are determined, it is easy to compute the overlap area of these halftone spots geometrically. In particular, circles and squares are convex shapes, thus their intersection with other circles or squares also generates a convex region. Neighboring vertices of an intersection region are always connected to each other by either a straight line or a circular arc depending on the shape of its constituent halftone spots. Thus, an intersection region may be composed of a polygon, circular segments, or both.

For the purpose of illustration, we show the intersection of three halftone spots (two circles and a square) in Fig. 12.

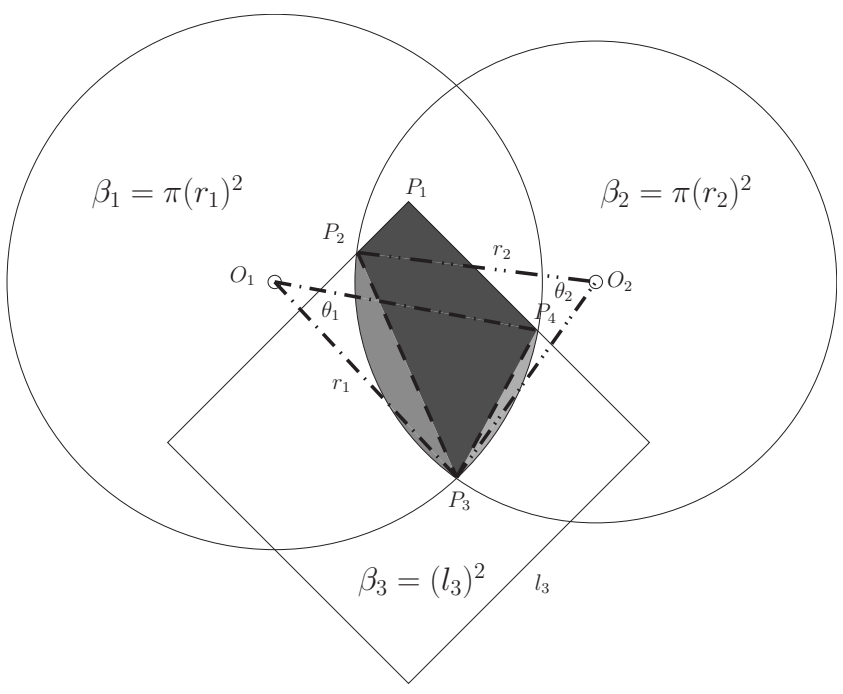

Fig. 12 An example of three intersecting halftone spots showing computation of areas that contribute to $\beta_{1}, \beta_{2}, \beta_{3}$, and $\beta_{123}$.
In this figure, the intersection region is formed by the polygon $P_{1} \rightarrow P_{2} \rightarrow P_{3} \rightarrow P_{4} \rightarrow P_{1}$ and two circular segments attached to this polygon, whose secant lines are $\overline{P_{2} P_{3}}$ and $\overline{P_{3} P_{4}}$. Each of these regions is shown in Fig. 12 using different shades of gray. The areas for these regions are readily computed, from which, the overlap area is directly obtained by summing the three indicated areas together. Within the unit cell $\mathcal{U}_{123}$, there are multiple instances of halftone spots of separations 1,2, and 3. The aforementioned computation is repeated for each possible selection that incorporates a spot from each of these separations. The sum of the areas from these individual computations is divided by the area of the unit cell $\mathcal{U}_{123}$ to obtain $\beta_{123}$.

This geometric model does not have a resolution constraint and computes significantly faster than the pixelcounting approach. We quantify this computational advantage in Table 5 in Sec. 8.2.

\section{Model Parameters}

Parameter values necessary for an exercising model of Fig. 9 can be obtained from experimental data. We briefly outline this process here. The spectra of $2^{K}$ Neugebauer primaries can be directly measured from prints of patches of each of the primaries. Single-colorant ramps for each of the $K$ separations are used to relate the digital control values that drive the printer to the respective area coverages $\left\{\beta_{k}\right\}_{k=1}^{K}$. For a single-colorant halftone image $h_{k}(\mathbf{x})$, the fractional area coverage $\beta_{k}$ is derived from the Neugebauer estimate of the spectrum using a least-squares procedure. ${ }^{34}$ For a single-colorant image, Eq. (5) becomes

$R_{\text {avg }}^{1 / \gamma}(\lambda)-R_{P}^{1 / \gamma}(\lambda)=\beta_{k}\left(R_{k}^{1 / \gamma}(\lambda)-R_{P}^{1 / \gamma}(\lambda)\right)$,

where $R_{\text {avg }}(\lambda), R_{k}(\lambda)$, and $R_{P}(\lambda)$ are the reflective spectra of the halftone image, $k^{\prime}$ th colorant, and paper substrate, respectively. In vector form, this can be written as $\mathbf{r}_{\text {avg }}$ $-\mathbf{r}_{P}=\beta_{k}\left(\mathbf{r}_{k}-\mathbf{r}_{P}\right)$, where $\mathbf{r}_{a v g}$ is a column vector of samples 
Table 1 CMYK halftone periodicities for the digital conventional configuration (for printer addressability $600 \times 600$ dpi; Ipi=lines per inch).

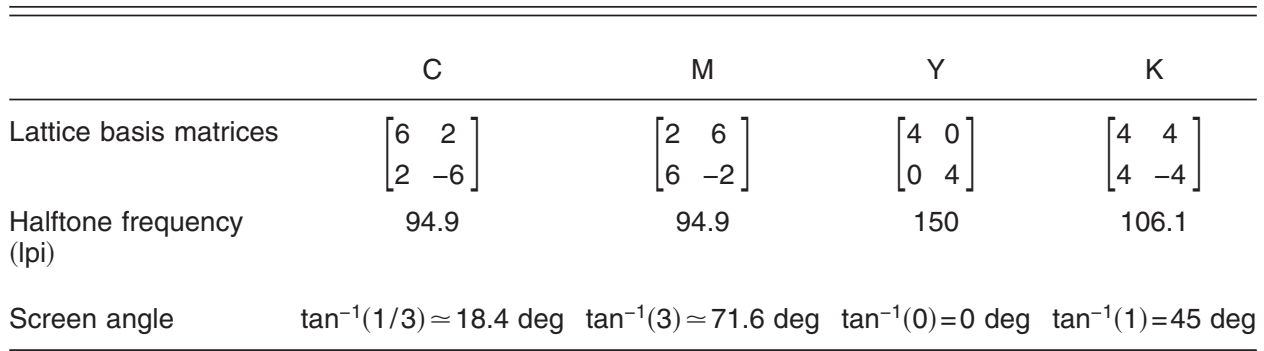

of $R_{\text {avg }}(\lambda) \frac{1}{\gamma}$ (at different values of the wavelength $\lambda$ ) and the other vectors are similarly the collections of samples of corresponding spectra. In this convention, the least-squares estimate of $\beta_{k}$ is obtained as

$\beta_{k}=\frac{\left(\mathbf{r}_{a v g}-\mathbf{r}_{P}\right)^{T}\left(\mathbf{r}_{k}-\mathbf{r}_{P}\right)}{\left\|\mathbf{r}_{k}-\mathbf{r}_{P}\right\|^{2}}$.

The previous procedure requires the value of the YN coefficient $\gamma$. The optimal value of $\gamma$ is selected from a set of candidate values by determining the value that minimizes the average mean-square error between the predicted reflectance value in Eq. (5) and the measured reflectance values over the complete set of colorant ramps.

\section{Simulation and Experimental Results}

Our experimental setup used a xerographic CMYK printer with an addressability of $4800 \times 600$ dpi [For convenience, we will state our lattice basis matrices in terms of a printer having identical addressability of $600 \mathrm{dpi}$ along each of the two orthogonal directions. Equivalent numbers for the $4800 \times 600$ dpi case are readily obtained by multiplying the first-rows of the basis matrices by a factor of 8 corresponding to the ratio of addressabilities in the horizontal and vertical directions]. The 16 Neugebauer primaries for the printer were measured from printed targets. Digital halftone screens with angular orientations close to the conventional analog screens were used as the primary halftones for our study although alternative halftone configurations were also investigated. The lattice periodicities for these halftones are specified by the basis matrices listed in Table 1 . These orthogonal screens are oriented as $C$ at $\tan ^{-1}(1 / 3)$ $\simeq 18.4 \mathrm{deg}, \mathrm{M}$ at $\tan ^{-1}(3) \simeq 71.6 \mathrm{deg}, \mathrm{Y}$ at $\tan ^{-1}(0)=0 \mathrm{deg}$, and $\mathrm{K}$ at $\tan ^{-1}(1)=45 \mathrm{deg}$. These angles are chosen to approximate the conventional 30 -deg difference between the frequency vectors of analog $\mathrm{C}, \mathrm{M}$, and $\mathrm{K}$ screens shown in Fig. 4(b). Thus, this configuration is referred to as the digital conventional configuration throughout this section. In addition to this configuration, we also explore two-colorant dot-on-dot/dot-off-dot halftone configurations. ${ }^{4}$ These configurations employ the same lattice periodicity for the twocolorant separations and maximize/minimize the overlap between the halftone spots of the two separations, respectively. The dot-on-dot configuration is obtained by using the same threshold function for the two separations. If the threshold function for one of the separations is displaced by half a period, a corresponding dot-off-dot configuration is obtained. The latter thus represents a misregistered version of the former. This specific halftone configuration and misregistration typically leads to the largest change in color. ${ }^{1,35}$ It is, therefore, particularly helpful for studying the effects of parameters other than the halftone periodicities. In our experiments, the basis matrix corresponding to the black (K) separation of Table 1 is used when referring to dot-ondot and dot-off-dot geometries.

As outlined in Sec. 6, single-colorant ramps of the C, M, $\mathrm{Y}$, and $\mathrm{K}$ colorants that ranged in area coverage from 0 to $100 \%$ were printed and utilized to estimate the relationship of digital CMYK values to the colorant fractional areas $\left\{\beta_{k}\right\}_{k=1}^{4}$. The YN parameter $\gamma$ was estimated as 1.4 and used throughout for the digital conventional halftone configuration. Color computations were performed using the CIE D50 standard illuminant ${ }^{30}$ and with a white point corresponding to the unprinted paper.

We carry out several simulations in which we investigate the change in average halftone color due to interseparation misregistration as a function of various experimental parameters. Specifically, we consider the impact of optical dot gain, colorant combinations, periodicity of the individual halftone separations, colorant area coverages, and misregistration amounts. We present results that explore various subsets of these factors. In doing so, we focus our attention on two-screen combinations because this allows us to explore the remaining parameter space more comprehensively and present results visually. We also consider specific questions of interest for three-screen CMK combinations toward the end of this section.

\subsection{Optical Dot Gain}

We consider the impact of optical dot gain on color misregistration sensitivity by evaluating color shifts (using the methodology of Fig. 9) for different values of the YN parameter $\gamma$. In general, an increase in the optical dot gain parameter $\gamma$ tends to reduce the magnitude of misregistration-induced color shifts. This is in agreement with physical intuition. As the optical dot gain increases, there is greater mixing of light entering the paper through the differently colored regions corresponding to the various Neugebauer primaries. This mixing reduces the dependence of the average spectrum/color on interseparation misregistration.

In Fig. 13, we illustrate this dependence on $\gamma$ for the sensitive CM dot-on-dot halftone configuration under the situation, where the fractional area coverages $\beta_{1}$ and $\beta_{2}$ 


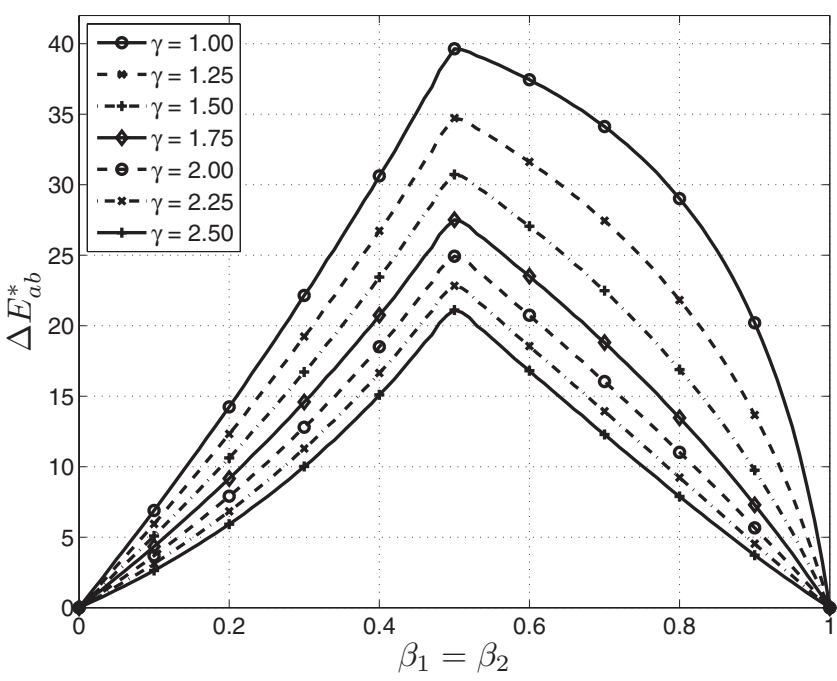

Fig. 13 Effect of optical dot gain on the amount of change in average color between the dot-on-dot and dot-off-dot configurations of $C$ $(k=1)$ and $\mathrm{M}(k=2)$ colorants when both colorants have equal fractional area coverages.

corresponding to the $\mathrm{C}$ and $\mathrm{M}$ separations are equal. The abscissa of the graph in Fig. 13 represents this fractional area coverage and the ordinate represents the change in color in $\Delta E_{a b}^{*}$ units produced when one of the separations is displaced by half the lattice period (producing a dot-off-dot configuration). The different plots on the graph represent the color change due to this misregistration for different values of the $\mathrm{YN}$ parameter $\gamma$. Note that for a given area coverage, as $\gamma$ increases, the amount of color shift reduces. This effect is quite significant when an increase in $\gamma$ from 1.0 to 2.5 reduces the worst case misregistration-induced color shift from 40 to approximately 21 in $\Delta E_{a b}^{*}$ units. Also, in the absence of YN correction $(\gamma=1)$, the corresponding curve in this figure is highly asymmetric with respect to the midtone gray level 0.5. In particular, shadow tones are significantly more sensitive to misregistration than the symmetrically located values in the highlight tones (about 0.5). As the YN parameter $\gamma$ increases, these curves become more symmetric and the amount of color shifts are also reduced. By examining differences in Neugebauer primary areas and spectra, instead of the $\Delta E_{a b}^{*}$ difference in CIELAB coordinates, we see that the CIELAB cube-root nonlinearity is the primary source of the asymmetry, which is in effect compensated by the higher $\gamma$ values.

\subsection{Dependence on Colorant Combinations}

We investigate color misregistration sensitivities of the different two-colorant combinations by evaluating the misregistration-induced color shifts for the dot-on-dot halftone configuration. In our simulations, we set the fractional area coverages of each of the colorants equal to 0.5 and a misregistration displacement for one of the screens equal to half the lattice period (which produces the corresponding dot-off-dot configuration). This configuration is chosen because it yields the largest (worst case) misregistrationinduced color shift for the $\mathrm{CM}$ colorant pair for all values of $\gamma$ in Fig. 13. All possible colorant pairs are considered. For each of these pairs, the average color is computed for the aforementioned perfectly registered and misregistered configurations. The color differences between the two configurations are computed in the individual CIELAB coordinates and in overall $\Delta E_{a b}^{*}$ units. These are listed in Table 2. It is clear that YK is the most and CY is the least sensitive colorant combination to misregistration in terms of the amount of color change. The MK combination, however, is the most sensitive one in terms of the magnitude of changes in the $L^{*}$ and $a^{*}$ coordinates. When different halftone geometries of these colorant combinations are tested (at different area coverages), the relative sensitivities for the different colorants maintain the observed ordering. Therefore, it would be advantageous to use less misregistration sensitive geometries for YK and MK colorant combinations. The relative sensitivities for colorant pairs observed in our simulations are in agreement with intuition developed from the registration insensitivity of colorants with nonoverlapping spectral bands (see Sec. 4.1). The CY combination has low sensitivity because cyan and yellow colorants have the least overlap in their spectral absorptance. Because black absorbs strongly in all regions of the spectrum, all combinations involving $\mathrm{K}$ demonstrate high sensitivity.

Table 2 Effect of colorant combinations on the amount of color change between the dot-on-dot and dot-off-dot halftone image of two colorants when both colorants have a fractional area coverage of 0.5 .

\begin{tabular}{lcccc}
\hline \hline Colorant Pair & $\Delta L^{*}$ & $\Delta a^{*}$ & $\Delta b^{*}$ & $\Delta E_{a b}^{*}$ \\
\hline CM & -18.81 & 17.96 & -20.50 & 33.12 \\
CY & -3.13 & -3.63 & 11.19 & 12.17 \\
CK & -28.53 & -22.48 & -32.09 & 48.47 \\
MY & -3.52 & 1.82 & 33.20 & 33.44 \\
MK & -31.28 & 47.18 & -7.92 & 57.16 \\
YK & -4.24 & -4.94 & 66.07 & 66.39 \\
\hline \hline
\end{tabular}


Table 3 Maximum amount of color change (in $\Delta E^{*}$ units) and misregistration sensitivity metric ( $\left.\zeta\right)$ for different geometries of $C$ ( $\left.k=1\right)$ and $\mathrm{M}$ $(k=2)$ colorants. (Lattice basis matrices are listed for printer addressability $600 \times 600 \mathrm{dpi}$; Ipi=lines per inch.)

\begin{tabular}{|c|c|c|c|c|c|c|c|c|c|c|c|c|}
\hline \multirow{3}{*}{$\frac{\text { Row }}{1}$} & \multicolumn{2}{|c|}{ Lattice Basis Matrices } & \multirow[b]{2}{*}{$\mathbf{V}_{12}=\mathbf{V}_{\Lambda_{1} \cap \Lambda_{2}}$} & \multirow[b]{2}{*}{$V_{\Lambda_{1}+\Lambda_{2}}$} & \multicolumn{2}{|c|}{ Frequencies (Ipi) } & \multicolumn{2}{|c|}{ Angles (deg) } & \multirow[b]{2}{*}{$\beta_{1}$} & \multirow[b]{2}{*}{$\beta_{2}$} & \multirow[b]{2}{*}{$\operatorname{Max} . \Delta E_{a b}^{*}$} & \multirow[b]{2}{*}{$\zeta_{12}$} \\
\hline & $\mathbf{v}_{1}$ & $\mathbf{v}_{2}$ & & & $f_{1}$ & $f_{2}$ & $\phi_{1}$ & $\phi_{2}$ & & & & \\
\hline & {$\left[\begin{array}{cc}4 & 4 \\
4 & -4\end{array}\right]$} & {$\left[\begin{array}{cc}4 & 4 \\
4 & -4\end{array}\right]$} & {$\left[\begin{array}{cc}4 & 4 \\
4 & -4\end{array}\right]$} & {$\left[\begin{array}{cc}4 & 4 \\
4 & -4\end{array}\right]$} & 106.1 & 106.1 & 45 & 45 & 0.50 & 0.50 & 31.35 & 1 \\
\hline 2 & {$\left[\begin{array}{cc}4 & 0 \\
0 & -4\end{array}\right]$} & {$\left[\begin{array}{cc}4 & 4 \\
4 & -4\end{array}\right]$} & {$\left[\begin{array}{cc}4 & 4 \\
4 & -4\end{array}\right]$} & {$\left[\begin{array}{cc}4 & 0 \\
0 & -4\end{array}\right]$} & 150 & 106.1 & 0 & 45 & 0.50 & 0.78 & 15.55 & 2 \\
\hline 3 & {$\left[\begin{array}{cc}8 & 8 \\
8 & -8\end{array}\right]$} & {$\left[\begin{array}{cc}4 & 4 \\
4 & -4\end{array}\right]$} & {$\left[\begin{array}{cc}8 & 8 \\
8 & -8\end{array}\right]$} & {$\left[\begin{array}{cc}4 & 4 \\
4 & -4\end{array}\right]$} & 53 & 106.1 & 45 & 45 & 0.88 & 0.58 & 7.96 & 4 \\
\hline 4 & {$\left[\begin{array}{cc}6 & 2 \\
2 & -6\end{array}\right]$} & {$\left[\begin{array}{cc}4 & 0 \\
0 & -4\end{array}\right]$} & {$\left[\begin{array}{cc}8 & 4 \\
4 & -8\end{array}\right]$} & {$\left[\begin{array}{cc}2 & 2 \\
2 & -2\end{array}\right]$} & 94.9 & 150 & 18.4 & 0 & 0.50 & 0.78 & 3.44 & 10 \\
\hline 5 & {$\left[\begin{array}{cc}6 & 2 \\
2 & -6\end{array}\right]$} & {$\left[\begin{array}{cc}4 & 4 \\
4 & -4\end{array}\right]$} & {$\left[\begin{array}{cc}12 & 4 \\
4 & -12\end{array}\right]$} & {$\left[\begin{array}{cc}2 & 2 \\
2 & -2\end{array}\right]$} & 94.9 & 106.1 & 18.4 & 45 & 0.91 & 0.89 & 2.16 & 20 \\
\hline 6 & {$\left[\begin{array}{cc}4 & 2 \\
2 & -4\end{array}\right]$} & {$\left[\begin{array}{cc}2 & 4 \\
4 & -2\end{array}\right]$} & {$\left[\begin{array}{cc}10 & 0 \\
0 & -10\end{array}\right]$} & {$\left[\begin{array}{cc}2 & 0 \\
0 & -2\end{array}\right]$} & 134.2 & 134.2 & 26.6 & 63.4 & 0.55 & 0.55 & 2.21 & 25 \\
\hline 7 & {$\left[\begin{array}{cc}6 & 2 \\
2 & -6\end{array}\right]$} & {$\left[\begin{array}{cc}2 & 6 \\
6 & -2\end{array}\right]$} & {$\left[\begin{array}{cc}10 & 10 \\
10 & -10\end{array}\right]$} & {$\left[\begin{array}{cc}2 & 2 \\
2 & -2\end{array}\right]$} & 94.9 & 94.9 & 18.4 & 71.6 & 0.55 & 0.55 & 2.22 & 25 \\
\hline 8 & {$\left[\begin{array}{cc}8 & 2 \\
2 & -8\end{array}\right]$} & {$\left[\begin{array}{cc}2 & 8 \\
8 & -2\end{array}\right]$} & {$\left[\begin{array}{cc}34 & 0 \\
0 & -34\end{array}\right]$} & {$\left[\begin{array}{cc}2 & 0 \\
0 & -2\end{array}\right]$} & 72.8 & 72.8 & 14 & 76 & 0.88 & 0.88 & 0.25 & 289 \\
\hline
\end{tabular}

\subsection{Impact of Halftone Geometry}

We next consider the impact of halftone geometry (lattice) on color misregistration sensitivity. For this purpose, we use the CM colorant pair because it exhibits significant sensitivity to misregistration in all three of the CIELAB coordinates. A number of lattice basis matrices are chosen for $\mathrm{C}$ and $\mathbf{M}$ separations, which are listed as $\mathbf{V}_{1}$ and $\mathbf{V}_{2}$, respectively, in Table 3. Basis matrices for the intersection lattice $\Lambda_{1} \cap \Lambda_{2}$ and the sum lattice $\Lambda_{1}+\Lambda_{2}$ are also listed in the table. For each pair of lattices, the maximum possible color shift due to misregistration is determined by a computational search over the possible values of the colorant areas and displacements. The corresponding colorant area coverages $\beta_{1}, \beta_{2}$, and the largest amount of the color shift obtained at these coverages are indicated in Table 3. Invariably the displacement producing the largest color shift was close to half a period of the sum lattice $\boldsymbol{\Lambda}_{1}+\boldsymbol{\Lambda}_{2}$. As expected, the largest amount of color shift is observed for the dot-on-dot geometry (corresponding to row 1 of Table 3), whose average color is known as extremely sensitive to interseparation misregistration. Relatively smaller color shifts are observed for rotated halftone geometries. The last column of the table lists a misregistration sensitivity metric for two-colorant halftone combinations that we describe subsequently in Sec. 8.1.

\subsection{Dependence on Colorant Area Coverages}

The dependence of misregistration-induced color shift on the colorant area coverages is examined next. Because the dependence on colorant area coverage exhibits significant variations under changes in halftone geometry, we consider it for the two-colorant dot-on-dot geometry and for each of the distinct pairs of lattice combinations for the digital conventional CMYK screen set. This latter set of combinations corresponds to rows 2 (YK), $4(\mathrm{CY}), 5(\mathrm{CK})$, and $7(\mathrm{CM})$ of Table 3.

For each of the colorant pairs, we fixed the interseparation misregistration to half a period of the sum of the two lattices and determined the color shift caused by this misregistration for different colorant area coverages $\beta_{k_{1}}, \beta_{k_{2}}$ for the two colorants. The plots in Fig. 14 present the results of these computations, where a CM combination was used for the dot-on-dot case [in Fig. 14(a)] [For the dot-on-dot configuration geometry, the shape of the plot in Fig. 14(a) remains largely unchanged for different colorant choices, only the magnitudes of the shifts vary]. Observe that the dynamic range of variation in Figs. 14(b) to 14(f) is much smaller than the range in Figs. 14(a) and 14(g): as expected the digital conventional geometry exhibit significantly less sensitivity to misregistration than the dot-on-dot configuration except for the YK pair. Also, the completely different topography of the surface plots in Figs. 14(a) to 14(g) demonstrate that the dependence of misregistration sensitivity on area coverage varies considerably with halftone geometry. The behavior for the digital conventional geometry indicates that the color change caused by misregistration does not vary unimodally with the colorant areas.

\subsection{Dependence on Amount of Displacement Misregistration}

With improving technologies for interseparation registration, ${ }^{36-38}$ only small displacements may actually be 


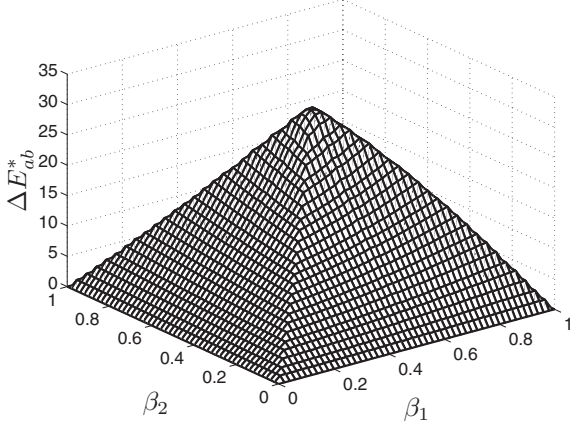

(a) Dot-on-dot CM

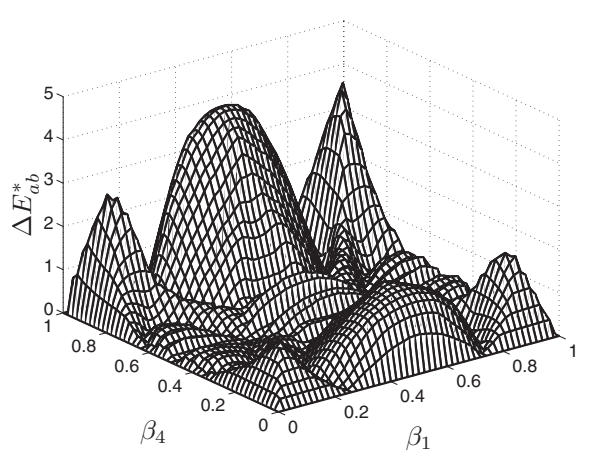

(d) Digital conventional CK

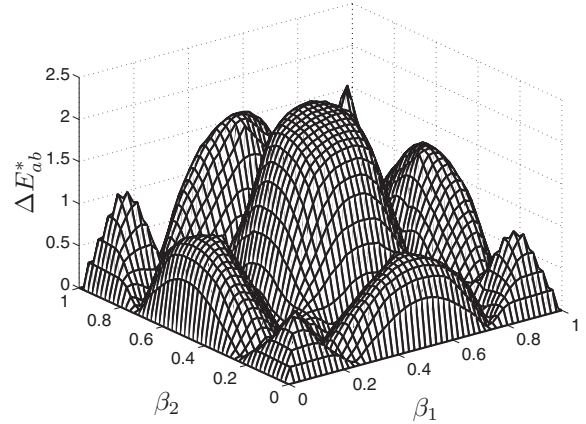

(b) Digital conventional CM

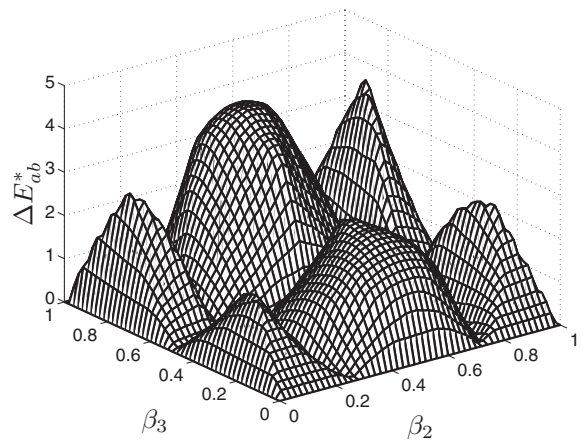

(e) Digital conventional MY

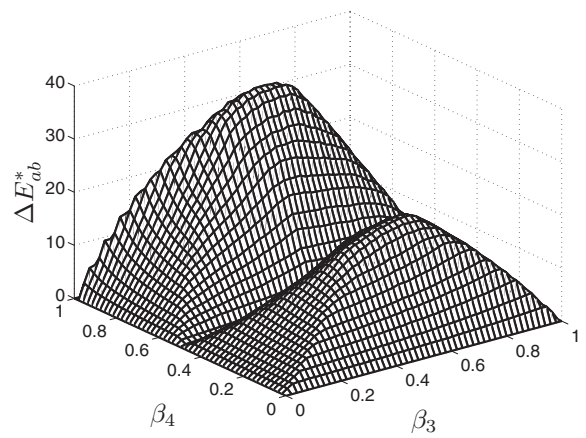

(g) Digital conventional YK

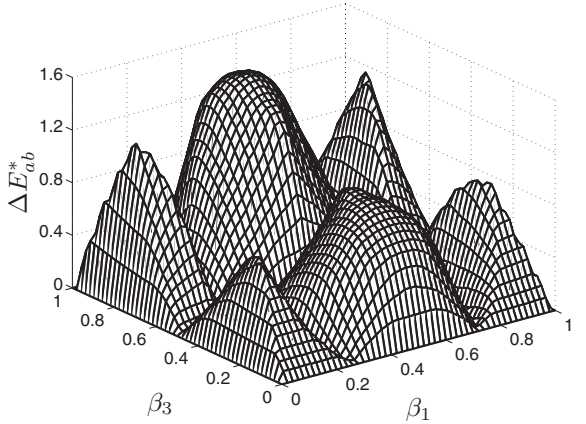

(c) Digital conventional CY

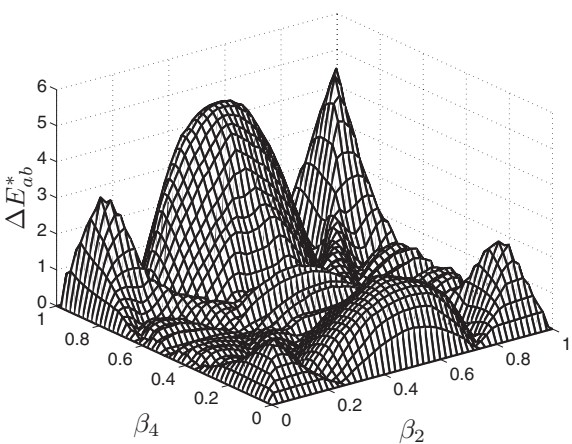

(f) Digital conventional MK

Fig. 14 Amount of color change (in $\Delta E_{a b}^{\star}$ units) between a perfectly registered and a misregistered composite halftone image of two colorants as a function of the fractional area coverages of $C(k=1)$, $\mathrm{M}(k=2), \mathrm{Y}(k=3)$, and $\mathrm{K}(k=4)$ colorants. In each configuration, $k_{2}$ 'th separation is misregistered by a half period of the sum of the lattices in the misregistered halftone image. Note the differences in the dynamic ranges of the different subfigures.

encountered in practice making the worst case computations of Table 3 overly pessimistic. We therefore consider the dependence of interseparation displacement amount on the misregistration-induced color shift.

To explore how the misregistration-induced color shift varies with different interseparation displacements, we once again consider the two-colorant CM dot-on-dot configuration, and each of the distinct colorant pairs for the digital conventional CMYK screen set. For these seven configurations, we set the fractional area coverages of each of these colorants to the area coverages corresponding to the highest peaks observed in Fig. 14. Figures 15(a) to $15(\mathrm{~g})$ show plots of the magnitude of color shift (in $\Delta E_{a b}^{*}$ units) as a function of the interseparation displacement coordinates $\Delta x_{k_{2}}$ and $\Delta y_{k_{2}}$ of the $k_{2}$ 'th separation along the $x$ and $y$ directions, respectively.

Note that the dynamic range of variations and $x$ and $y$ axes in these plots are different. As in Fig. 14, the twocolorant CM dot-on-dot and the digital conventional YK pair demonstrate high sensitivity to misregistration, whereas other colorant pairs from the digital conventional CMYK screen set are less sensitive. However, a larger amount of misregistration is needed to reach the highest peaks of Figs. 15(a) and $15(\mathrm{~g})$ than the remaining digital conventional pairs shown in Figs. 15(b) to 15(f). These results can also help establish misregistration tolerances 


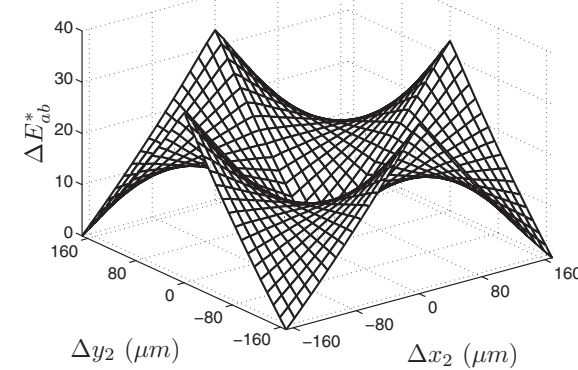

(a) Dot-on-dot CM

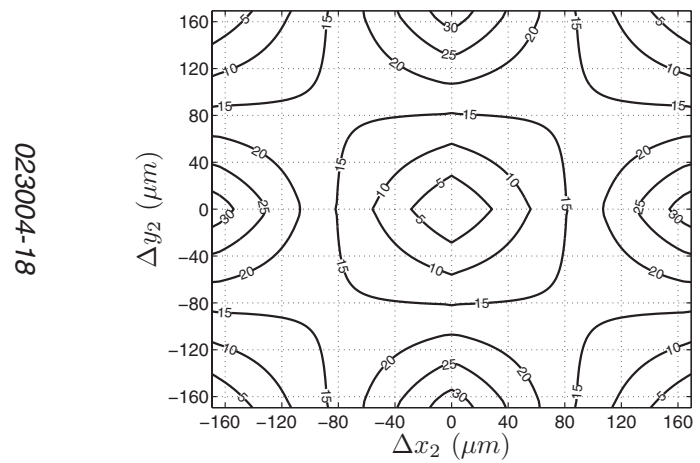

(h) Dot-on-dot CM

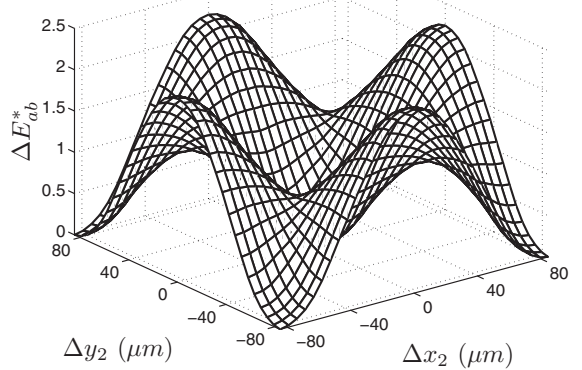

(b) Digital conventional CM

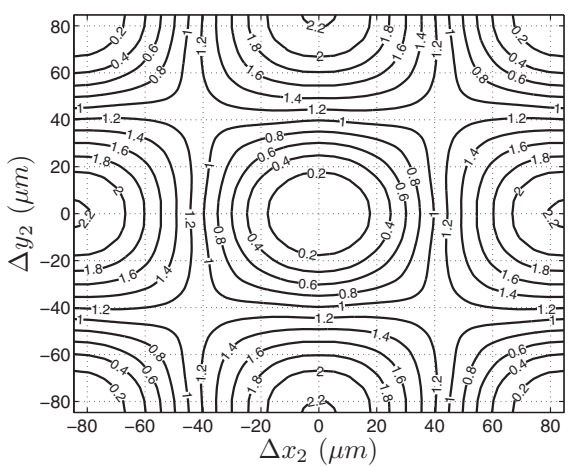

(i) Digital conventional CM

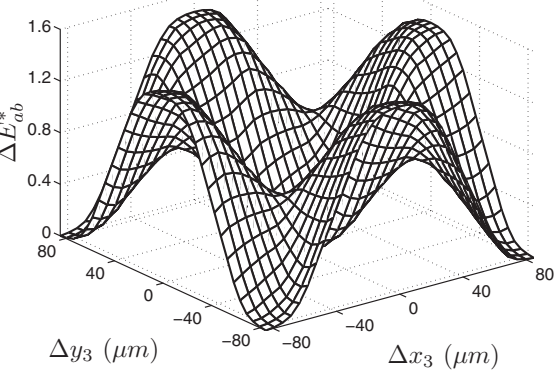

(c) Digital conventional CY

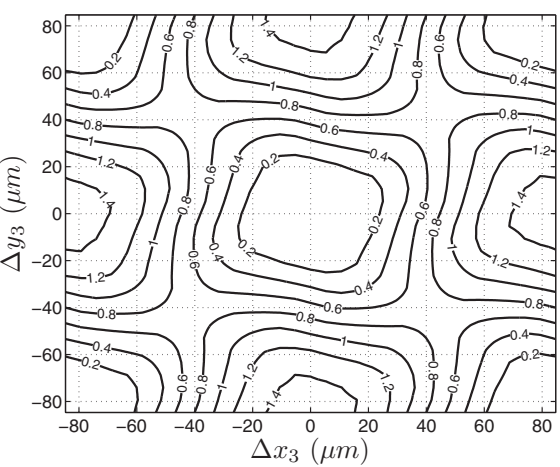

(j) Digital conventional CY

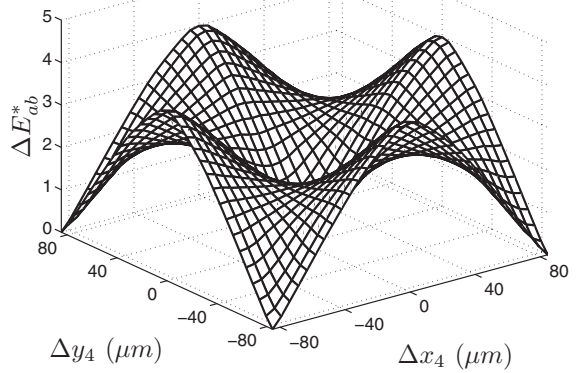

(d) Digital conventional CK

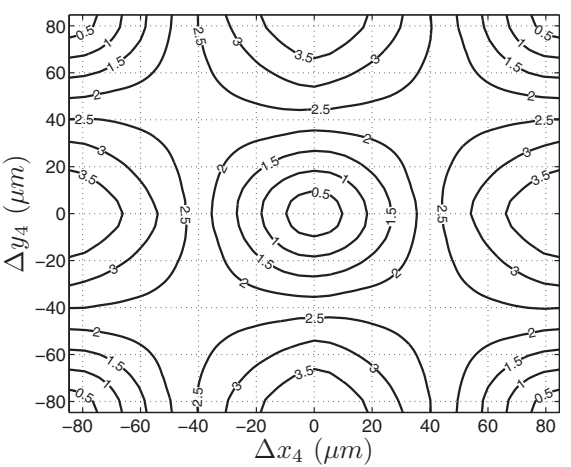

(k) Digital conventional CK

Fig. 15 Amount of color change (in $\Delta E_{a b}^{*}$ units) between a perfectly registered and a misregistered composite halftone image of distinct pairs of $C(k=1), M(k=2), Y(k=3)$, and $\mathrm{K}(k=4)$ colorants as a function of the displacement amounts in one of the separations. In each configuration, fractional area coverages of the colorants are set to the area coverages corresponding to the peaks observed in Fig 14. Panels (a) to (g) represent the color errors as surface plots for the two-colorant CM dot-on-dot and the two-colorant digital conventional halftone configurations of distinct colorant pairs. Panels $(\mathrm{h})$ to $(\mathrm{n})$ are contour maps corresponding to the topographies in (a) to (g), respectively. Labels on the contour lines in (h) to (n) plots correspond to the amount of color change (in $\Delta E^{*}$ units). Thus, contours represen " that is less than the label for the contour. Note that misregistration amounts are varied within the unit
cell of the sum lattice $\Lambda_{k_{1}}+\Lambda_{k_{2}}$ of each configuration and given in micrometers. Note the differences in the dynamic ranges of the different subfigures. 


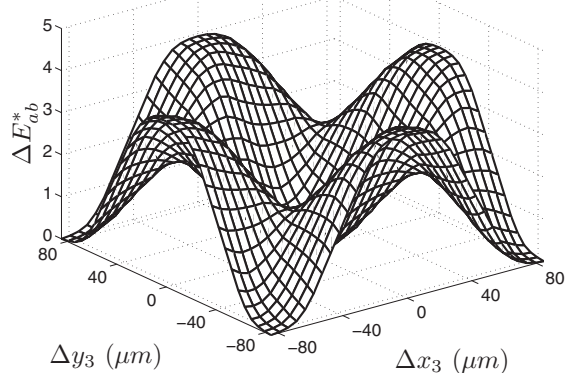

(e) Digital conventional MY

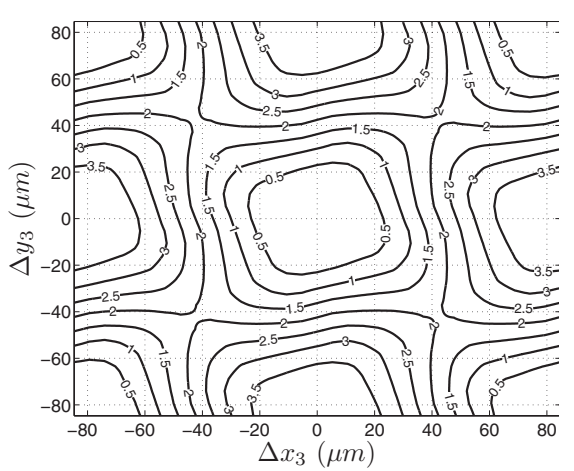

(1) Digital conventional MY

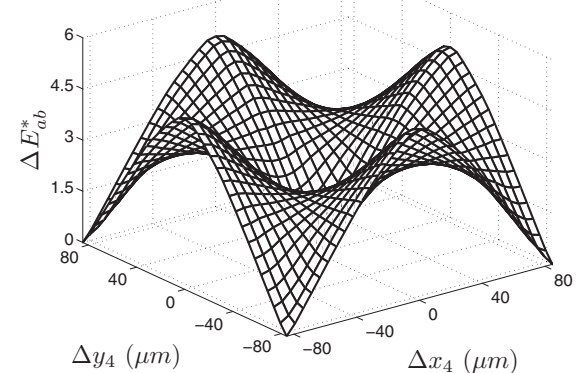

(f) Digital conventional MK

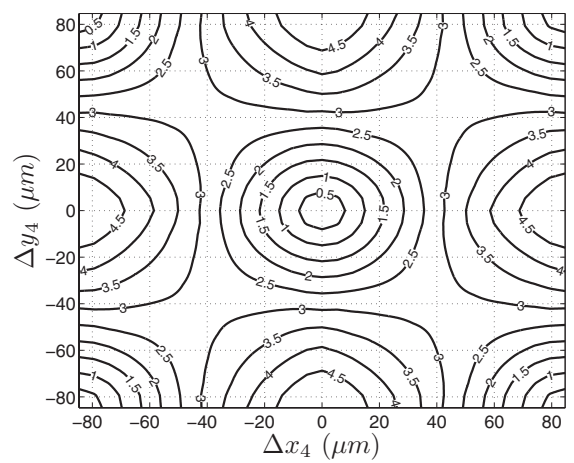

(m) Digital conventional MK

Fig. 15 (Continued).

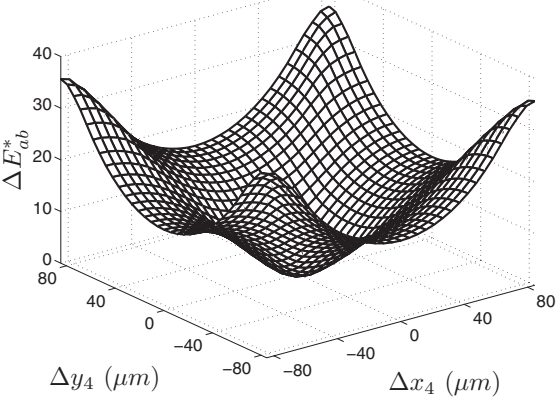

(g) Digital conventional YK

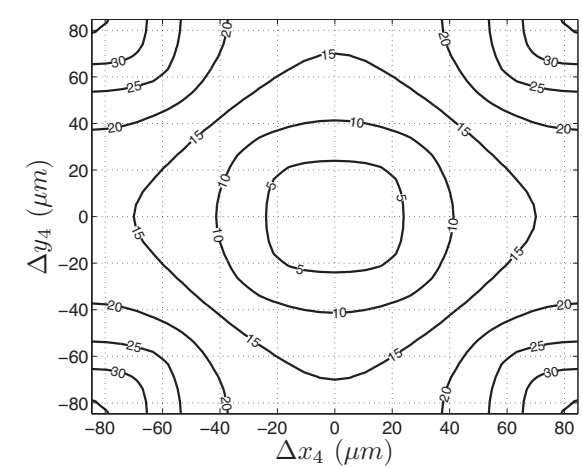

(n) Digital conventional YK 

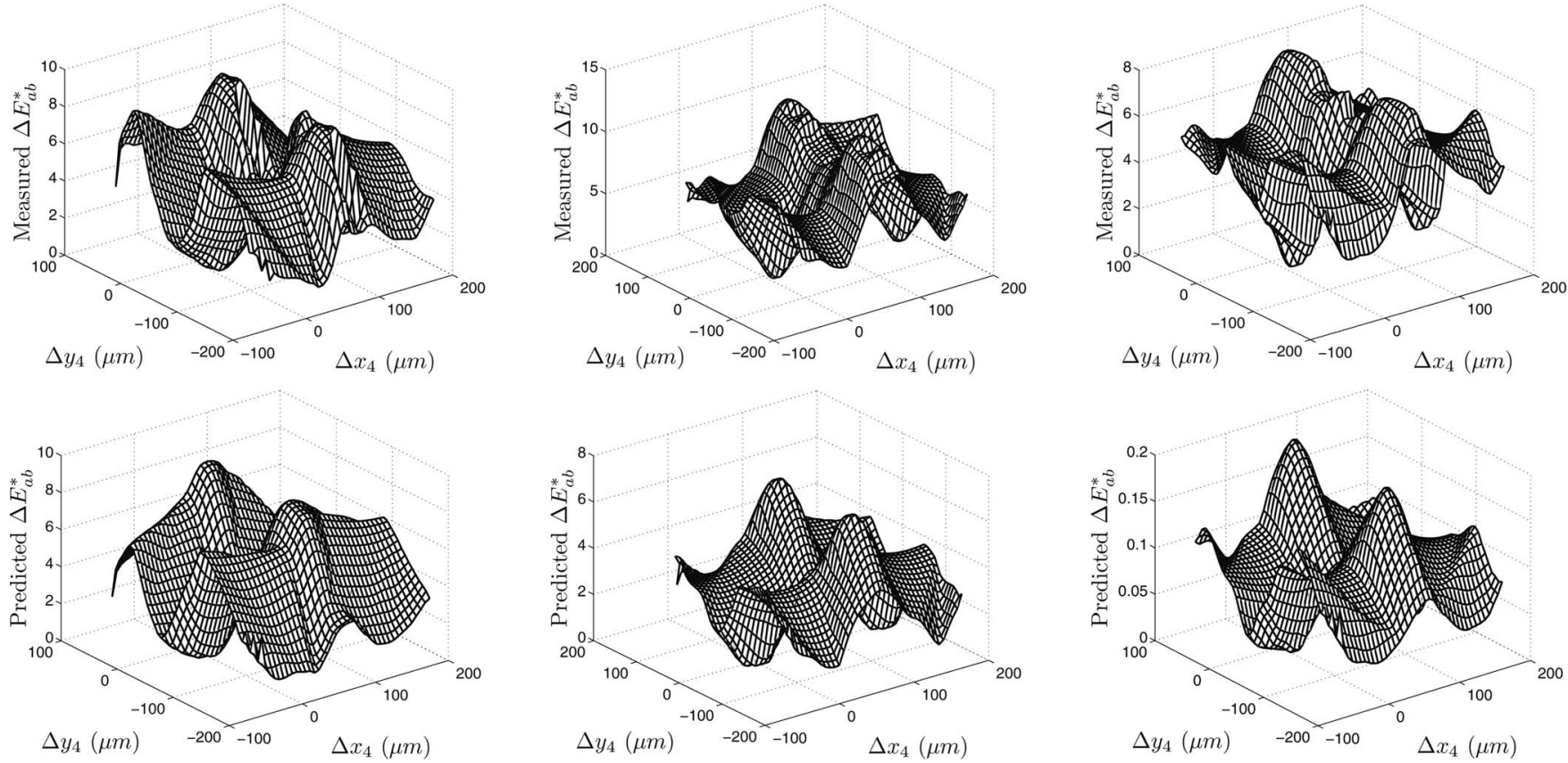

(a) $\beta_{3} \approx \beta_{4} \approx 0.3$

(b) $\beta_{3} \approx \beta_{4} \approx 0.4$

(c) $\beta_{3} \approx \beta_{4} \approx 0.5$
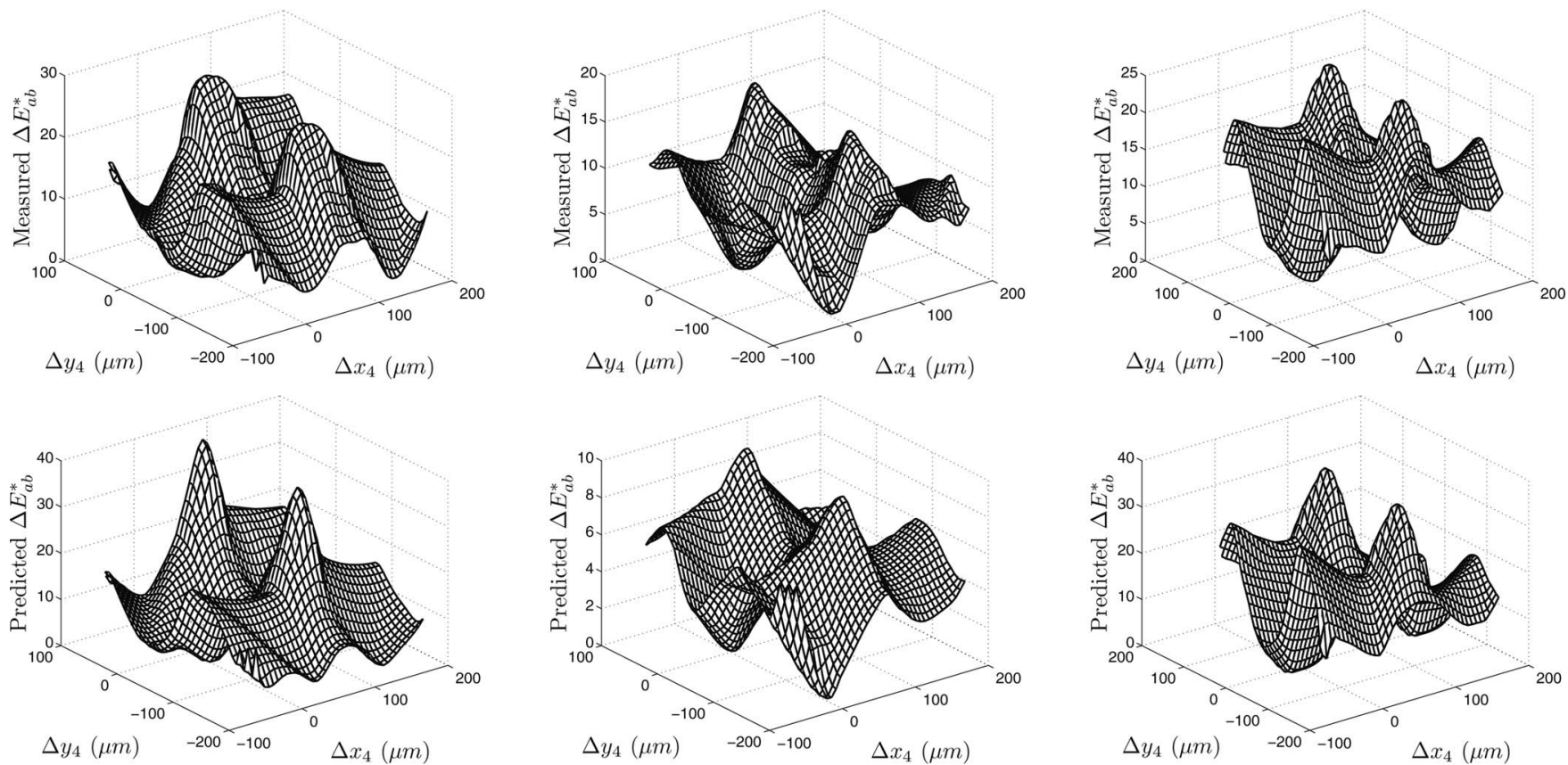

(d) $\beta_{3} \approx 0.5$ and $\beta_{4} \approx 0.3$

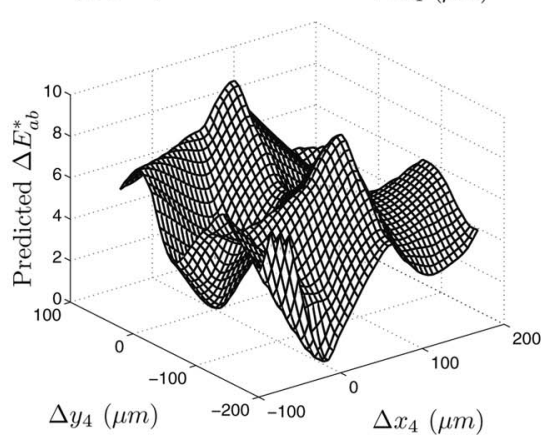

(e) $\beta_{3} \approx 0.6$ and $\beta_{4} \approx 0.4$

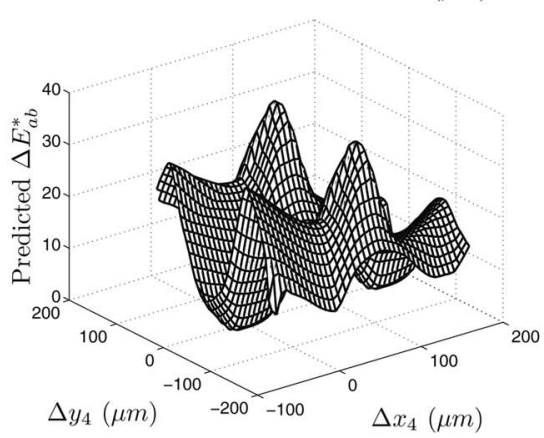

(f) $\beta_{3} \approx \beta_{4} \approx 0.7$

Fig. 16 Comparison of experimentally measured data versus simulation results for $Y(k=3)$ and $K$ $(k=4)$ combination in the digital conventional orientation. Note the differences in the dynamic ranges of the different subfigures.

based on known color tolerances. We illustrate this in Figs. $15(\mathrm{~h})$ to $15(\mathrm{n})$, which show the contour maps of the topographies shown in Figs. 15(a) to 15. The contours about the origin $\left(\Delta x_{k_{2}}=0, \Delta y_{k_{2}}=0\right)$ in Figs. 15(h) to $15(\mathrm{n})$ represent the region of registration displacements for which the color shift is less than or equal to the $\Delta E_{a b}^{*}$ tolerance indicated on the contour lines.

\subsection{Model Predictions Versus Experimentally Measured Color Shifts}

Experimental validation of the model predictions is challenging for multiple reasons. First, spatially varying interseparation misregistration is inevitable in the printing systems we use for any experimental study. Thus, it is not feasible to obtain prints with a specific desired amount of 
interseparation misregistration. Second, our model for color halftones incorporates only the first-order effects of geometry and optics and provides predictions of color change due to interseparation misregistration based on these effects. Actual printing systems, however, include additional physical effects that influence the color of the printed output and the color shifts caused by interseparation misregistration. In the present investigation, we do not attempt to model these additional physical effects, which usually depend on the printing technology used. As a result, the model predictions of color shifts can be masked by these other effects, particularly in scenarios where the predicted color changes are relatively small.

We addressed the first of the above challenges by using methods that allow us to estimate the interseparation misregistration in our printed samples. ${ }^{39}$ We addressed the issue of potential masking of predicted effects by other physical phenomena by focusing the experiments on scenarios where the predictions of misregistration-induced color shift were rather large (and therefore less likely to be masked). Specifically, we selected the YK two-colorant combination from the digital conventional CMYK screen set. As can be seen from Figs. 14(g) and 15(g), the predicted color shifts due to misregistration are rather large for this colorant pair in this halftone configuration.

For our experiment, we generated targets with combinations of yellow (Y) and black (K) colorants, where the area coverages for the two colorants ranged from approximately 0.3 through 0.8 . Each target was composed of multiple patches. The colorant area coverages for the two colorants were constant over the patches in a target. The individual $\mathrm{Y}$ and $\mathrm{K}$ separations in a patch were halftoned using the corresponding screens for the digital conventional geometry and a different interseparation misregistration was deliberately introduced in each of the patches, except one. These deliberate misregistrations covered the values allowed by the device addressability. In addition, to allow an estimation of the uncontrolled (nondeliberate) interseparation misregistration as a function of spatial location, we included on each printed page a grid of fiducial markers. ${ }^{39}$

From the printed targets, average color values were measured for each patch. The interseparation misregistration for each patch was also estimated by adding together the deliberate misregistration introduced in the target and the estimate of the uncontrolled misregistration in the printer, where the latter was obtained from the fiducial markers using the method of Ref. 39. Predictions of average color were made for each patch using the simulation framework of Fig. 9 with the estimated interseparation misregistration for the patch and the corresponding colorant coverage values. Color differences were then computed between each of the patches in a target and the first patch in the target, for both the measured and the predicted values. Figure $16 \mathrm{com}-$ pares these measured versus predicted color shifts for the YK colorant combination (which exhibited the most sensitivity to misregistration).

From the plots, several observations can be made. First, on an absolute basis, the agreement between the predictions and the measured values is rather poor. As mentioned earlier, this is due to physical effects that our simple model ignores. On the other hand, we observe that the relative shapes of the plots for the predictions and the measure-

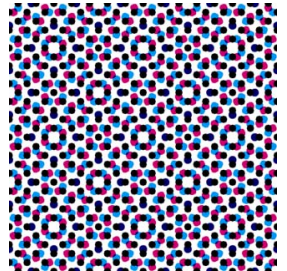

Clear-centered rosette

(a)

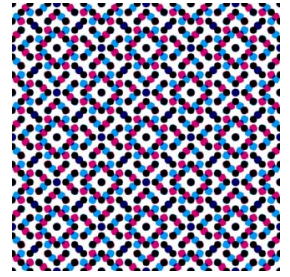

Dot-centered rosette

(b)
Fig. 17 Rosettes in digital conventional CMK halftone screens. (Color online only.)

ments are in general agreement. This indicates that the proposed methodology is still useful in halftone design, where often only the relative amounts of errors are of interest.

Specifically, the plots of Fig. 16(c) demonstrate rather poor agreement between the simulations and experiments. This is because for an area coverage of 0.5 , for the ideal square dot shapes, these two halftone configurations are theoretically misregistration invariant. They correspond exactly to the configuration shown in Fig. 7 and discussed in Sec. 4.3. In practice, however, by examining the prints under a microscope, we observed that in this case, the change in misregistration caused a significant change in the shape and sizes of the dots of the separations (possibly due to xerographic development interactions).

\subsection{Three-Colorant Combinations}

For combinations having more than two colorants, a graphical presentation of the color misregistration sensitivity becomes infeasible due to the large number of variables (which cannot be readily represented as 2D or 3D perspective plots). We therefore considered two specific questions of interest, for three-colorant CMK combinations. First, we consider equi-colorant-area configurations $\left(\beta_{k_{1}}=\beta_{k_{2}}=\beta_{k_{3}}\right)$ and evaluate the change in color caused by a half period displacement in each of the separations, which transforms the microstructure from the clear-centered rosette to the dot-centered rosette (As shown in Sec. 4.4, smaller displacements may also produce the same microstructure). Figure 17 illustrates specific instances of these configurations for our digital conventional CMK screens.

Figure 18(a) shows a plot of the magnitude of color shift between these two extreme rosettes as a function of the fractional area for the three colorants. For the purpose of comparison, we also show in Fig. 18(b) the corresponding plot obtained for the case of conventional CMK analog screens that was previously investigated by Daels et al. ${ }^{19}$ (Conventional screens require $\infty$ measurement aperture. Because this is impossible to realize in simulations, we choose the regions corresponding to the images shown in Fig. 5 as the unit cell of the overlay, which is almost periodic within the overall image). Recall that the two rosettes for this case were shown earlier in Fig. 5. A comparison of Figs. 18(a) and 18(b) show that with respect to misregistration-induced color shifts, digital CMK screens surprisingly behave quite differently than the conventional analog screens they are designed to approximate. Specifically, the conventional analog configuration shows a unimodal behavior with variation in colorant area coverage, whereas multiple 


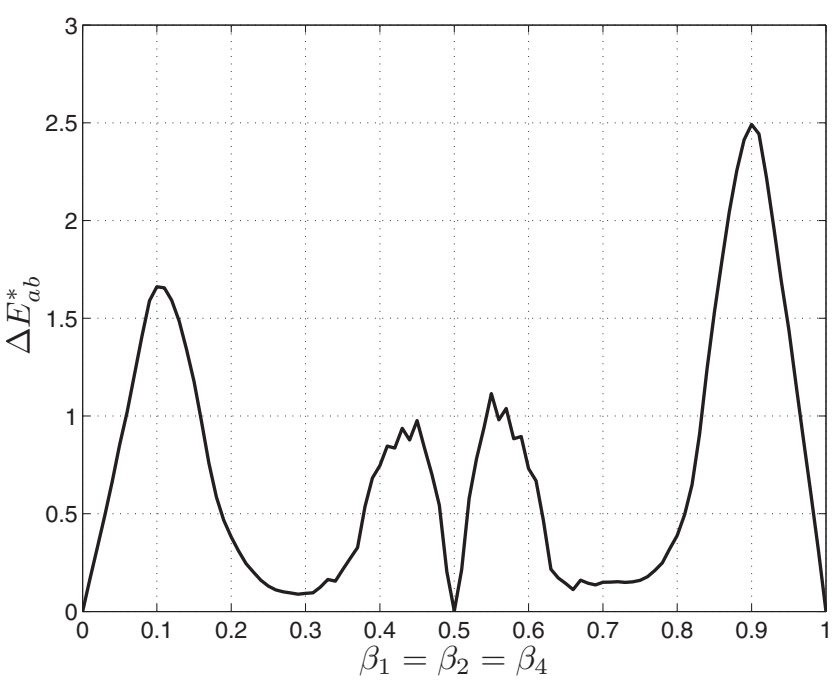

Digital conventional

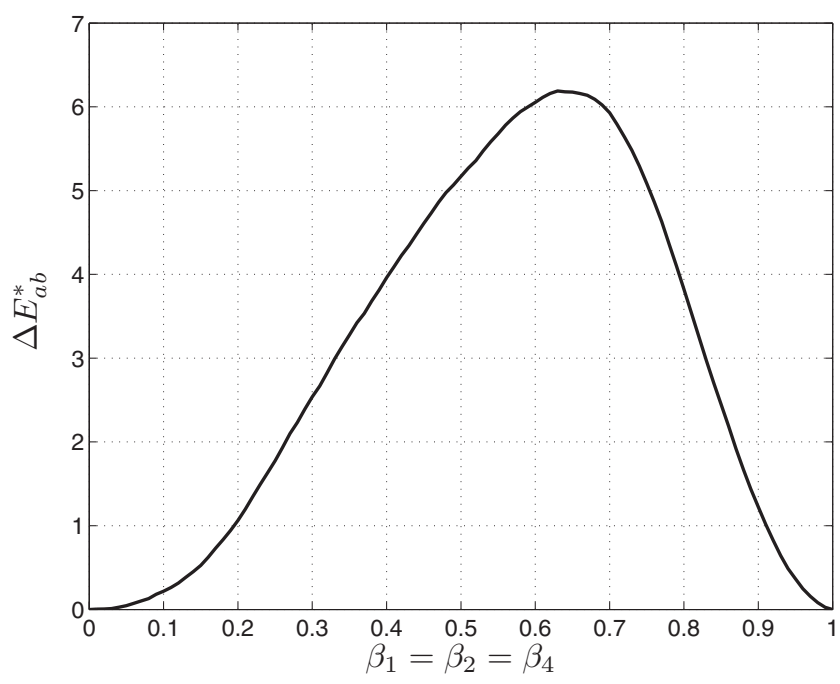

Conventional

Fig. 18 Amount of color change (in $\Delta E^{*}$ units) between clear-centered and dot-centered rosettes of $\mathrm{C}(k=1), \mathrm{M}(k=2)$, and $\mathrm{K}(k=4)$ halftones when all separations have equal fractional area coverage.

maxima and minima are observed for the digital screens. The maximum color shift for the two cases (across the range of colorant coverages) are also quite different with the maximal color shift for the digital configuration (2.5 $\Delta E_{a b}^{*}$ units) being significantly lower than the maximal color shift for the analog conventional (6.2 $\Delta E_{a b}^{*}$ units).

Next, we tested the conjecture whether the two extremes of clear-centered and dot-centered rosettes for equal colorant configurations represent the worst case scenarios. Our simulations indicate that these do not in fact represent the situation with largest color shifts; it is possible to obtain color shifts around $7 \Delta E_{a b}^{*}$ units for the conventional and $5.3 \Delta E_{a b}^{*}$ units for the digital conventional configurations between a perfectly registered and a misregistered composite CMK halftone image [These corresponded to $\beta_{1}=0.55$, $\beta_{2}=0.63, \beta_{4}=0.59$ for the conventional and $\beta_{1}=0.09, \beta_{2}$ $=0.53, \beta_{4}=0.89$ for the digital conventional $\mathrm{C}(k=1), \mathrm{M}$ $(k=2), \mathrm{K}(k=4)$ screen configurations].

\section{Discussion}

In this work, we characterized the sensitivity of clustereddot color halftones to interseparation misregistration using a simple representation that combines a periodic lattice representation for individual halftones with an overlay-based Neugebauer model. Although the representation allowed us to characterize misregistration sensitivity as a function of several factors, it ignores several additional physical effects that come into play in practical printing systems:

1. Actual halftone dots in printing systems do not display a "hard profile" corresponding to the on/off states. Instead, actual dots show variation in colorant density particularly close to the edges. More sophisticated models (e.g., Ref. 40) can incorporate these effects, but these models tend to be much more computation intensive. Our simpler model allows us to more readily explore the parameter space that influences misregistration-induced color shifts.
2. In some printing systems, intercolorant suppression may be encountered. ${ }^{6,41}$ For instance, within an offset printing process, spatial positions on paper that are covered with wet ink arrayed in a halftone pattern can be less efficient in trapping ink than dry locations on paper. Thus, the presence of a first halftone image separation modulates, or suppresses, a second halftone image separation. Analogous suppression occurs in electrophotographic processes in steps such as development and transfer. This suppression phenomenon is not considered explicitly in our work. However, we note that the Neugebauer primary reflectances are measured from prints that also incorporate this suppression ensuring that it is accounted for to a first-order approximation.

3. As described in Sec. 4.2, nonsingular halftone configurations are insensitive to interseparation misregistration. With this motivation, one might wish to search for a nonsingular lattice configuration for the colorants. Although this may be feasible for an analog printing device, addressable device locations of a digital printer are confined to a rectilinear grid and the singularity condition is always satisfied for any two periodicity lattices. Thus, digital clustered-dot color halftones are typically sensitive to interseparation misregistration.

4. In Sec. 4.3, we illustrated that misregistration invariance may be obtained for specific spot functions. The practical significance of this result is limited by the fact that the spot function conditions cannot be universally met for a halftone screen when arbitrary gray levels are considered. However, the result may be useful in the design of tints and gradations of tints, where the use of a threshold halftone screen function is not mandated.

5. Our computation of the colorant overlap areas assumes a "measurement aperture" that corresponds to a unit cell of the intersection lattice of the colorant 
Oztan, Sharma, and Loce: Misregistration sensitivity in clustered-dot color halftones

Table 4 Maximum misregistration for some printing technologies (see Ref. 42) (Ipi=lines per inch).

\begin{tabular}{lccc}
\hline \hline Method of Printing & Substrate & Frequency (Ipi) & Max. Misregistration $(\mu \mathrm{m})$ \\
\hline Sheet-fed offset & Gloss coated & 150 & 80 \\
Sheet-fed offset & Uncoated & 150 & 80 \\
Web-fed offset & Gloss coated & 150 & 100 \\
Web-fed offset & Uncoated commercial & 133 & 130 \\
Web-fed offset & Newsprint & 100 & 150 \\
Flexography & Coated & 133 & 150 \\
Flexography & Newsprint & 100 & 200 \\
Flexography & Kraft (corrugated, other) & 65 & 250 \\
Screen printing (wet-on-wet) & Fabric & any & 0 \\
Screen printing (dried) & Paper, fabric, other & 100 & 150 \\
Gravure & Gloss coated & 150 & 80 \\
\hline \hline
\end{tabular}

lattices involved (or an integer multiple thereof). The size of this unit cell is not constrained in our analysis. In practice, however, the aperture size of a color measurement device is finite. If the size of a unit cell of the aforementioned intersection lattice is large in comparison to the measurement aperture, practically one may observe registration sensitivity for configurations that are insensitive under the current analysis. However, these configurations would also typically demonstrate perceptible local variations in halftone color even with perfect interseparation misregistration.

6. Throughout this paper, interseparation misregistration is assumed to be a constant displacement of a separation with respect to another that does not vary spatially. In practice, however, there might be spatial variations in misregistration within a separation. In addition, there might also be small angular misregis-

Table 5 Average computation time required to compute Neugebauer primary fractional areas by using pixel-counting and proposed method. A 2.8-GHz Intel Pentium 4 PC system with 2 GBytes of main memory running on Microsoft Windows XP was used for timing simulations in MATLAB 7.0.1.

\begin{tabular}{ccccccc}
\hline \hline & & \multicolumn{2}{c}{ Pixel-Count Method } & & \multicolumn{2}{c}{ Algebraic Method } \\
$\beta_{1}$ & $\beta_{2}$ & $t_{\text {avg }}(\mathrm{sec})$ & $\Delta E_{a b}^{*}$ & & $t_{\text {avg }}(\mathrm{sec})$ & $\Delta E_{a b}^{*}$ \\
\hline 0.4 & 0.4 & 5.88 & 1.74 & & 0.22 & 1.63 \\
0.3 & 0.3 & 5.88 & 0.35 & & 0.13 & 0.46 \\
0.4 & 0.3 & 5.88 & 0.69 & & 0.33 & 0.60 \\
\hline \hline
\end{tabular}

tration, which might lead to color moiré. These types of misregistrations are beyond the scope of this paper and not considered in our analysis.

7. We noted in our simulation results in Sec. 7.1 that the misregistration-induced color shifts are reduced as the optical dot gain increases. It is known that low frequency halftone screens suffer less from optical dot gain than high frequency halftone screens. ${ }^{6}$ Therefore, one may expect that the low frequency halftone screens exhibit more sensitivity to misregistration than the high frequency halftone screens. However, at the same time, because the periods of the low frequency halftones are larger than the periods of the high frequency halftones, the worst case misregistration displacement for the former are larger in magnitude than the worst case misregistration displacements for the latter. These worst case displacements may not actually be attained in practical systems. Table 4 shows examples of maximum expected misregistration amounts of some printing technologies, circa $1995 .{ }^{42}$ Current technologies for xerographic printing can further confine registration errors around the 40 to $50-\mu \mathrm{m}$ range, which can still yield relatively large amounts of color shifts as shown in Figs. 15(h) to 15(n).

\subsection{A Misregistration Sensitivity Metric for Clustered-Dot Halftone Geometries}

The goal of halftone screen design is to ensure the printed image does not exhibit any objectionable color moiré and color sensitivity to interseparation misregistration and achieves a large color gamut while keeping the parameters such as lattice periodicities within the specifications. Some configurations, such as dot-off-dot halftones, may not exhibit any color moiré and offer larger gamut than other 
configurations, such as rotated halftone screens; however, these can be extremely sensitive to interseparation misregistration. ${ }^{35}$ Thus, despite their limitations, halftone periodicities that are less sensitive to interseparation misregistration are preferable and commonly used in practice. Using computer-aided design software, it is possible to find configurations subject to certain frequency, gamut, and moiré constraints. ${ }^{43}$ In addition, if available, a misregistration sensitivity constraint could also play a significant role in selecting color halftone configurations, which satisfy the aforementioned requirements. For this purpose, we suggest a readily computable metric for the color misregistration sensitivity of an overlay of two-colorant halftone separations based on their geometry alone.

Equation (29) for the spatial domain computation of area coverages of colorant combinations averages the areas of every possible spot intersection within $\mathcal{U}_{\mathbf{k}(\mathbf{c})}$. As the number of distinct terms contributing to this averaging increases, we expect that fractional areas (thus, average halftone color) exhibit less sensitivity to interseparation misregistration. Thus, for a two-colorant halftone overlay, the number of possible spot overlaps contributing to the averaging may be thought of as a simple metric for the misregistration sensitivity. This number is bounded by the pairwise displacements of the halftone spots within $\mathcal{U}_{\mathbf{k}(\mathbf{c})}$. Consider an overlay of two halftone separations with lattices $\boldsymbol{\Lambda}_{k_{1}}$ and $\boldsymbol{\Lambda}_{k_{2}}$. As Eq. (29) suggests, the number of distinct terms included in the averaging is equal to the number of elements of the sum lattice $\boldsymbol{\Lambda}_{k_{1}}+\boldsymbol{\Lambda}_{k_{2}}$ in $\mathcal{U}_{k_{1} k_{2}}$. Let $\mathcal{U}_{\boldsymbol{\Lambda}_{k_{1}}+\boldsymbol{\Lambda}_{k_{2}}}$ represent a unit cell of the lattice $\boldsymbol{\Lambda}_{k_{1}}+\boldsymbol{\Lambda}_{k_{2}}$. We define a metric $\zeta_{k_{1} k_{2}}$ indicating denseness of $\mathcal{U}_{\boldsymbol{\Lambda}_{k_{1}}+\boldsymbol{\Lambda}_{k_{2}}}$ in $\mathcal{U}_{k_{1} k_{2}}$ as the total number of unit cells $\mathcal{U}_{\Lambda_{k_{1}}+\Lambda_{k_{2}}}$ of the sum lattice that are needed to tile a unit cell $\mathcal{U}_{k_{1} k_{2}}$ of the intersection lattice. The metric $\zeta_{k_{1} k_{2}}$ can be computed as the ratio between the areas of $\mathcal{U}_{k_{1} k_{2}}$ and $\mathcal{U}_{\boldsymbol{\Lambda}_{k_{1}}+\boldsymbol{\Lambda}_{k_{2}}}$ and is referred to as the index of $\boldsymbol{\Lambda}_{k_{1}}+\boldsymbol{\Lambda}_{k_{2}}$ in $\boldsymbol{\Lambda}_{k_{1}} \cap \boldsymbol{\Lambda}_{k_{2}} \cdot{ }^{28}$

The quantitative amount of misregistration-induced color shift is not determined by the geometry alone and depends, among other factors, on the spectral characteristics of the colorants, shapes of the halftone spots, and the interseparation misregistration amounts. Thus, we cannot expect the metric $\zeta$ to directly indicate the amount of color shift. Nevertheless, for a given colorant pair, this metric can be expected to be related to the maximum achievable color shift for the corresponding halftone geometry. Table 3 shows the maximum achievable color shift and the metric $\zeta$ for the overlay of $\mathrm{C}$ and $\mathrm{M}$ halftone screens in different halftone geometries. One can see that the metric offers good correlation with the maximum color shift for the geometry. The dot-on-dot halftone configuration (row 1) exhibits the most sensitivity to misregistration and has the lowest value of $\zeta$. In general, the configurations with smaller values for the maximum color shift have higher values of $\zeta$, maintaining a monotonic relation with one exception.

\subsection{Computational Considerations}

In Sec. 5.2, we proposed an approximation that allows analytic computation of colorant overlap areas with the objec- tive of reducing computations. We next quantify the computational advantage and concurrently evaluate the accuracy of the approximation. Table 5 indicates the average computation time required to calculate the Neugebauer primary areas for a two-colorant digital conventional CM halftone configuration for the pixel-counting and analytic computation methods. For the pixel-counting method, we set the unit cell size of the intersection lattice to 1280 $\times 1280$ pixels to reduce the pixelation effects. To evaluate the performance of the analytic computation method with different spot functions, we consider all possible scenarios: square-square, circle-circle, and square-circle spot intersections and these results for these scenarios are listed in the respective order in the table.

It can be seen that analytic computation significantly reduces the computation time compared to the pixelcounting technique with a good approximation to the amount of color shift. This computational advantage is particularly useful in simulations, which require long computation times. However, pixel-counting technique is particularly useful to evaluate the misregistration-induced color shifts in arbitrary lattice periodicity configurations such as uniform rosette halftones ${ }^{44}$ or frequency vector sharing methods, where nonorthogonal halftone geometries are employed.

\section{Conclusion}

In this paper, we examined interseparation misregistrationinduced color shifts in periodic clustered-dot color halftones. Thorough analysis of the problem requires understanding of both spectral and spatial interactions between the colorant separations of the printed image. We present a mathematical characterization of the problem that reveals scenarios under which color invariance to misregistration can be achieved depending on the colorant spectra, separation periodicities, halftone spot functions, and displacement amounts. Specifically, our analysis revealed conditions on halftone spot shapes and misregistration amounts under which invariance can be achieved, in spite of having colorant spectra and individual separation halftone periodicities that would otherwise indicate sensitivity. For simulations, we presented a computationally efficient hybrid analyticalnumerical framework that allows us to obtain quantitative estimates of color shifts due to misregistration as a function of spectral and spatial characteristics of the separations. Our experimental results indicate that several physical effects that are not included within our model can have a significant influence on the color shifts due to interseparation misregistration. As a result, the model's predictions are not quantitatively accurate though they are in qualitative agreement with experimental data when the predicted variations are large enough to avoid masking by the other factors.

\section{Appendix A: Fourier Transform of Halftone Overlay $h_{\mathrm{k}(\mathrm{c})}(\mathbf{x})$}

The convolution of two functions $H_{1}(\mathbf{u})$ and $H_{2}(\mathbf{u})$ can be written in integral form as 


$$
\begin{aligned}
\left(H_{1} * H_{2}\right)(\mathbf{u}) & =\int H_{1}\left(\mathbf{u}_{1}\right) H_{2}\left(\mathbf{u}-\mathbf{u}_{1}\right) \mathrm{d} \mathbf{u}_{1} \\
& =\iint H_{1}\left(\mathbf{u}_{1}\right) H_{2}\left(\mathbf{u}_{2}\right) \delta\left[\mathbf{u}-\left(\mathbf{u}_{1}+\mathbf{u}_{2}\right)\right] \mathrm{d} \mathbf{u}_{1} \mathrm{~d} \mathbf{u}_{2} .
\end{aligned}
$$

$$
\left(H_{1} * \cdots * H_{K}\right)(\mathbf{u})=\int \cdots \int H_{1}\left(\mathbf{u}_{1}\right) \ldots H_{K}\left(\mathbf{u}_{K}\right) \delta\left(\mathbf{u}-\sum_{k=1}^{K} \mathbf{u}_{k}\right) \mathrm{d} \mathbf{u}_{1} \ldots \mathrm{d} \mathbf{u}_{K} .
$$

Equation (12) can be written using the individual colorant halftone separation model given in Eq. (13) as

$$
\begin{aligned}
H_{\mathbf{k}(\mathbf{c})}(\mathbf{u})= & \int \cdots \int H_{k_{1}}^{\left(\mathbf{d}_{k_{1}}\right)}\left(\mathbf{u}_{k_{1}}\right) \ldots H_{k_{m}}^{\left(\mathbf{d}_{k_{m}}\right)}\left(\mathbf{u}_{k_{m}}\right) \delta\left(\mathbf{u}-\sum_{k \in \mathbf{k}(\mathbf{c})} \mathbf{u}_{k}\right) \mathrm{d} \mathbf{u}_{k_{1}} \ldots \mathrm{d} \mathbf{u}_{k_{m}} \\
= & \int \cdots \int \prod_{k \in \mathbf{k}(\mathbf{c})}\left(\frac{1}{\left|\mathbf{V}_{k}\right|} S_{k}\left(\mathbf{u}_{k}\right) \exp \left(-2 \pi j \mathbf{d}_{k}^{T} \mathbf{u}_{k}\right) \sum_{\mathbf{n}_{k}} \delta\left(\mathbf{u}_{k}-\mathbf{W}_{k} \mathbf{n}_{k}\right)\right) \delta\left(\mathbf{u}-\sum_{l \in \mathbf{k}(\mathbf{c})} \mathbf{u}_{l}\right) \mathrm{d} \mathbf{u}_{k_{1}} \ldots \mathrm{d} \mathbf{u}_{k_{m}} \\
= & \prod_{k \in \mathbf{k}(\mathbf{c})}\left(\frac{1}{\left|\mathbf{V}_{\mathbf{k}}\right|} \sum_{\mathbf{n}_{k}} S_{k}\left(\mathbf{W}_{k} \mathbf{n}_{k}\right) \exp \left(-2 \pi j \mathbf{d}_{k}^{T} \mathbf{W}_{k} \mathbf{n}_{k}\right)\right) \delta\left(\mathbf{u}-\sum_{l \in \mathbf{k}(\mathbf{c})} \mathbf{W}_{l} \mathbf{n}_{l}\right)=\sum_{\mathbf{n}_{k_{1}}} \ldots \sum_{\mathbf{n}_{k_{m}}} \prod_{k \in \mathbf{k}(\mathbf{c})}\left(\frac{1}{\left|\mathbf{V}_{k}\right|} S_{k}\left(\mathbf{W}_{k} \mathbf{n}_{k}\right)\right. \\
& \left.\times \exp \left(-2 \pi j \mathbf{d}_{k}^{T} \mathbf{W}_{k} \mathbf{n}_{k}\right)\right) \delta\left(\mathbf{u}-\sum_{l \in \mathbf{k}(\mathbf{c})} \mathbf{W}_{l} \mathbf{n}_{l}\right) .
\end{aligned}
$$

\section{Appendix B: Invariant Misregistrations for K-Colorant Configuration}

Consider the summation in Eq. (16) for the scenario where $\mathbf{k}(\mathbf{c})=k_{1} k_{2} \ldots k_{m}$. Denoting

$\mathcal{S}_{\mathbf{k}(\mathbf{c})}=\left\{\left(\mathbf{u}_{1}, \mathbf{u}_{2}, \ldots, \mathbf{u}_{m}\right) \in \Lambda_{k_{1}}^{*} \times \Lambda_{k_{2}}^{*} \times \cdots \times \Lambda_{k_{m}}^{*} \mid \sum_{l} \mathbf{u}_{l}=\mathbf{0}\right\}$,

we can rewrite Eq. (16) as

$\beta_{\mathbf{k}(\mathbf{c})}=\frac{1}{\prod_{k \in \mathbf{k}(\mathbf{c})}\left|\mathbf{V}_{k}\right|} \sum_{\left(\mathbf{u}_{1}, \ldots, \mathbf{u}_{m}\right) \in \mathcal{S}_{\mathbf{k}(\mathbf{c})}} S_{k_{1}}\left(\mathbf{u}_{1}\right) S_{k_{2}}\left(\mathbf{u}_{2}\right) \ldots S_{k_{m}}\left(\mathbf{u}_{m}\right) \times \exp \left[-2 \pi j\left(\mathbf{d}_{k_{1}}^{T} \mathbf{u}_{1}+\mathbf{d}_{k_{2}}^{T} \mathbf{u}_{2}+\cdots+\mathbf{d}_{k_{m}}^{T} \mathbf{u}_{m}\right)\right]$.

It follows that for displacements for which the term

$\mathbf{d}_{k_{1}}^{T} \mathbf{u}_{1}+\mathbf{d}_{k_{2}}^{T} \mathbf{u}_{2}+\cdots+\mathbf{d}_{k_{m}}^{T} \mathbf{u}_{m}$

is integer-valued, the exponential becomes unity, which is identical to the perfectly registered case. Thus, $\mathbf{d}_{\mathbf{k}(\mathbf{c})}$ $=\left(\mathbf{d}_{k_{1}}, \ldots, \mathbf{d}_{k_{m}}\right)$ is an invariant displacement for the fractional area $\beta_{\mathbf{k}(\mathbf{c})}$ if the following condition holds

$$
\begin{aligned}
\forall\left(\mathbf{u}_{1}, \mathbf{u}_{2}, \ldots, \mathbf{u}_{m}\right) \in & \boldsymbol{\Lambda}_{k_{1}}^{*} \times \Lambda_{k_{2}}^{*} \times \cdots \times \Lambda_{k_{m}}^{*}, \\
& \ni \sum_{l} \mathbf{u}_{l}=\mathbf{0}, \\
& \sum_{l} \mathbf{d}_{k_{l}} \mathbf{u}_{l} \in \mathbb{Z} .
\end{aligned}
$$

Next, we show that (41) is equivalent to

$$
\left(\mathbf{d}_{k_{j}}-\mathbf{d}_{k_{i}}\right) \mathbf{u}_{j} \in \mathbb{Z}, \quad \forall \mathbf{u}_{j} \in \Lambda_{k_{j}}^{*} \cap \Lambda_{k_{i}}^{*},
$$

for all pairs $i \neq j$ in $\{1, \ldots, m\}$. To show that (41) implies (42), we rewrite $\sum_{l=1}^{m} \mathbf{u}_{l}=\mathbf{0}$ as $\mathbf{u}_{i}=-\sum_{j=1, j \neq i}^{m} \mathbf{u}_{j}$ and substitute in (41) to obtain

$$
\begin{gathered}
\sum_{\substack{j=1 \\
j \neq i}}^{m}\left(\mathbf{d}_{k_{j}}-\mathbf{d}_{k_{i}}\right)^{T} \mathbf{u}_{j} \in \mathbb{Z}, \quad \forall\left\{\mathbf{u}_{j}\right\}_{j \neq i}, \quad \ni \mathbf{u}_{j} \in \Lambda_{k_{j}}^{*}, \\
\text { and } \sum_{j \neq i} \mathbf{u}_{j} \in \Lambda_{k_{i}}^{*}
\end{gathered}
$$

Setting $\mathbf{u}_{j}=\mathbf{0}$ for all $j=1, \ldots, m, j \neq i, l$, we see that (43) implies $\left(\mathbf{d}_{k_{l}}-\mathbf{d}_{k_{i}}\right)^{T} \mathbf{u}_{j} \in \mathbb{Z}$, for all $\mathbf{u}_{l} \in \boldsymbol{\Lambda}_{k_{l}}^{*} \cap \boldsymbol{\Lambda}_{k_{i}}^{*}$. This establishes that (41) implies (42). To show (42) implies (41), we 
note that using Lemma 1 from Appendix $\mathrm{C}$, any $\left(\mathbf{u}_{1}, \mathbf{u}_{2}, \ldots, \mathbf{u}_{m}\right) \in \mathcal{S}_{\mathbf{k}(\mathbf{c})}$ can be decomposed into the form $\mathbf{u}_{i}=\sum_{j \neq i} \mathbf{u}_{i}^{j}, \quad i=1, \ldots, m, j=1, \ldots, m, j \neq i$ such that $\mathbf{u}_{i}^{j}$ $\in \boldsymbol{\Lambda}_{k_{i}}^{*} \cap \boldsymbol{\Lambda}_{k_{j}}^{*}$ and $\mathbf{u}_{i}^{j}=-\mathbf{u}_{j}^{i}$ [Note that when $\mathbf{V}_{i}^{-1} \mathbf{V}_{j}$ is a matrix of rational numbers, we can assume without loss of generality that $\boldsymbol{\Lambda}_{i}$ and $\boldsymbol{\Lambda}_{j}$ represent integer lattices]. Using this expression, we can write (40) as

$$
\begin{aligned}
\sum \mathbf{d}_{k_{i}}^{T} \mathbf{u}_{i} & =\mathbf{d}_{k_{1}}^{T}\left(\sum_{j \neq 1} \mathbf{u}_{1}^{j}\right)+\mathbf{d}_{k_{2}}^{T}\left(\sum_{j \neq 2} \mathbf{u}_{2}^{j}\right)+\cdots+\mathbf{d}_{k_{m}}^{T}\left(\sum_{j \neq m} \mathbf{u}_{m}^{j}\right) \\
& =\sum_{i=1}^{m-1} \sum_{j=i+1}^{m}\left(\mathbf{d}_{k_{i}}-\mathbf{d}_{k_{j}}\right)^{T} \mathbf{u}_{i}^{j} .
\end{aligned}
$$

Using the definition of the reciprocal lattice, if $\mathbf{d}_{k_{i}}-\mathbf{d}_{k_{j}}$ $\in\left(\Lambda_{k_{i}}^{*} \cap \Lambda_{k_{j}}^{*}\right)^{*}=\boldsymbol{\Lambda}_{k_{i}}+\boldsymbol{\Lambda}_{k_{j}}$ for all colorant pairs $k_{i} \neq k_{j}$ in $\mathbf{k}(\mathbf{c})$, then $\left(\mathbf{d}_{k_{i}}-\mathbf{d}_{k_{j}}\right)^{T} \mathbf{u}_{i}^{j}$ is integer-valued. This establishes that (42) implies (41). Therefore, $\mathbf{d}_{\mathbf{k}(\mathbf{c})}=\left(\mathbf{d}_{k_{1}}, \ldots, \mathbf{d}_{k_{m}}\right)$ is an invariant misregistration for the fractional area $\beta_{\mathbf{k}(\mathbf{c})}$ if

$\mathbf{d}_{k_{i}}-\mathbf{d}_{k_{j}} \in \Lambda_{k_{i}}+\Lambda_{k_{j}}$ for all pairs $k_{i} \neq k_{j}$ in $\left\{k_{1}, \ldots, k_{m}\right\}$.

The displacement misregistrations for which the complete set of areas $\left\{\beta_{\mathbf{k}(\mathbf{c})}\right\}_{\mathbf{c}}$ are invariant, define the invariant misregistrations for the general $K$ colorant case. From our analysis for a single colorant pair in Sec. 4.4, the twocolorant overlap area $\beta_{i j}$ is invariant if displacement misregistrations satisfy the condition $\mathbf{d}_{i}-\mathbf{d}_{j} \in \boldsymbol{\Lambda}_{i}+\boldsymbol{\Lambda}_{j}$. Thus, this condition must hold for each colorant pair $(i, j)$ to ensure that the corresponding colorant overlap areas are invariant. We demonstrated above that these sets of conditions on the pairwise displacements are in fact sufficient to ensure invariance of the fractional area $\beta_{\mathbf{k}(\mathbf{c})}$ for any colorant combination $\mathbf{k}(\mathbf{c})$. Thus, we see that misregistrations for which any combination of colorants is guaranteed to be invariant are characterized by

$\mathbf{d}_{i}-\mathbf{d}_{j} \in \boldsymbol{\Lambda}_{i}+\boldsymbol{\Lambda}_{j}$ for all pairs $i \neq j$ in $\{1, \ldots, K\}$.

\section{Appendix C: An Antisymmetric Decomposition}

Lemma 1 Suppose $\boldsymbol{\Lambda}_{1}, \boldsymbol{\Lambda}_{2}, \ldots, \boldsymbol{\Lambda}_{m}$ are integer lattices, that is, $\mathbf{\Lambda}_{i} \subseteq \mathbb{Z}^{2}, \quad i=1, \ldots, m$, and $\left(\mathbf{u}_{1}, \mathbf{u}_{2}, \ldots, \mathbf{u}_{m}\right) \in \boldsymbol{\Lambda}_{1} \times \boldsymbol{\Lambda}_{2}$ $\times \cdots \times \Lambda_{m}$ satisfy $\sum \mathbf{u}_{l}=\mathbf{0}$. Then, there exists $\mathbf{u}_{i}^{j}, \quad i$ $=1, \ldots, m, j=1, \ldots, m, j \neq i$ such that

$$
\begin{aligned}
& \text { 1. } \mathbf{u}_{i}^{j} \in \boldsymbol{\Lambda}_{i} \cap \boldsymbol{\Lambda}_{j} \\
& \text { 2. } \mathbf{u}_{i}=\sum_{\substack{j=1 \\
j \neq i \\
j \neq i}}^{j} \mathbf{u}_{i} \\
& \text { 3. } \mathbf{u}_{i}^{j}=-\mathbf{u}_{j}^{i} .
\end{aligned}
$$

Proof We shall establish this result by using induction on $m$.

First consider the case $m=2$. We have $\mathbf{u}_{1} \in \boldsymbol{\Lambda}_{1}, \mathbf{u}_{2} \in \Lambda_{2}$, and $\mathbf{u}_{1}+\mathbf{u}_{2}=\mathbf{0}$. It follows that $\mathbf{u}_{1}=-\mathbf{u}_{2}$. If we set $\mathbf{u}_{1}^{2}=\mathbf{u}_{1}$ and $\mathbf{u}_{2}^{1}=\mathbf{u}_{2}$, then $\mathbf{u}_{1}^{2} \in \Lambda_{1} \cap \Lambda_{2}$ and $\mathbf{u}_{2}^{1} \in \Lambda_{2} \cap \Lambda_{1}$, and $\mathbf{u}_{1}^{2}=-\mathbf{u}_{2}^{1}$. Thus, the result holds for $m=2$.
Next assuming the lemma holds for $m \leq(l-1)$, we consider the case $m=l$. We have $\sum_{i=1}^{l} \mathbf{u}_{i}=\mathbf{0}$ and $\mathbf{u}_{i} \in \boldsymbol{\Lambda}_{i}$ for $i$ $\in 1, \ldots, l$. Rewriting the above equation as

$\mathbf{u}_{l}=-\sum_{i=1}^{(l-1)} \mathbf{u}_{i}$

we observe that the left-hand side (LHS) lies in $\boldsymbol{\Lambda}_{l}$ and the right-hand side (RHS) lies in $\sum_{i=1}^{(l-1)} \boldsymbol{\Lambda}_{i}$. Thus, $\mathbf{u}_{l}$ $\in \boldsymbol{\Lambda}_{l} \cap\left(\sum_{i=1}^{(l-1)} \boldsymbol{\Lambda}_{i}\right)=\sum_{i=1}^{(l-1)}\left(\boldsymbol{\Lambda}_{l} \cap \boldsymbol{\Lambda}_{i}\right)$.

The last equality above follows from the distributivity of lattice intersection over summation for integer lattices. This distributivity follows from the distributivity of the least common right multiple $(\mathrm{lcrm})$ over the greatest common left divisor (gcld) for integer matrices and the fact that, for integer lattices the basis matrix for the intersection lattice $\boldsymbol{\Lambda}_{i} \cap \boldsymbol{\Lambda}_{j}$ is $\mathbf{V}_{\boldsymbol{\Lambda}_{i} \cap \boldsymbol{\Lambda}_{j}}=\operatorname{lcrm}\left(\mathbf{V}_{i}, \mathbf{V}_{j}\right)$ and the basis matrix for the summation lattice $\boldsymbol{\Lambda}_{i}+\boldsymbol{\Lambda}_{j}$ is $\mathbf{V}_{\boldsymbol{\Lambda}_{i}+\boldsymbol{\Lambda}_{j}}=\operatorname{gcld}\left(\mathbf{V}_{i}, \mathbf{V}_{j}\right)$ (see Ref. 27, pp. $35-38){ }^{45}$

Now, because $\mathbf{u}_{l} \in \sum_{i=1}^{(l-1)}\left(\boldsymbol{\Lambda}_{l} \cap \boldsymbol{\Lambda}_{i}\right)$, there exists $\mathbf{u}_{l}^{j}, j$ $=1, \ldots,(l-1)$, such that $\mathbf{u}_{l}^{j} \in \boldsymbol{\Lambda}_{l} \cap \boldsymbol{\Lambda}_{j}$ and $\mathbf{u}_{l}=\sum_{j=1}^{(l-1)} \mathbf{u}_{l}^{j}$. For $i=1, \ldots,(l-1)$, define $\mathbf{w}_{i}=\mathbf{u}_{i}+\mathbf{u}_{l}^{i}$. Then, $\mathbf{w}_{i} \in \Lambda_{i}$, for all $i$ $=1, \ldots, l$ and

$$
\begin{aligned}
\sum_{i=1}^{(l-1)} \mathbf{w}_{i} & =\sum_{i=1}^{(l-1)} \mathbf{u}_{i}+\sum_{i=1}^{(l-1)} \mathbf{u}_{l}^{i} \\
& =\sum_{i=1}^{l} \mathbf{u}_{i}=\mathbf{0} .
\end{aligned}
$$

Thus, by the induction hypothesis there exists $\mathbf{w}_{i}^{j}, i$ $=1, \ldots,(l-1), j=1, \ldots,(l-1), j \neq i$ such that $\mathbf{w}_{i}^{j} \in \boldsymbol{\Lambda}_{i} \cap \boldsymbol{\Lambda}_{j}$, $\mathbf{w}_{i}=\sum_{j=1, j \neq i}^{(l-1)} \mathbf{w}_{i}^{j}$, and $\mathbf{w}_{i}^{j}=-\mathbf{w}_{j}^{i}$. Define

$\mathbf{u}_{i}^{j}=\left\{\begin{array}{cl}\mathbf{w}_{i}^{j} & \text { for } i=1, \ldots,(l-1), j=1, \ldots,(l-1), j \neq i, \\ -\mathbf{u}_{l}^{i} & \text { for } j=l, i=1, \ldots,(l-1) .\end{array}\right.$

Then, $\mathbf{u}_{j}^{i} \in \boldsymbol{\Lambda}_{i} \cap \boldsymbol{\Lambda}_{j}$ and $\sum_{j=1, j \neq i}^{l} \mathbf{u}_{i}^{j}=\mathbf{u}_{i}$. The latter equation holds trivially for $i=l$. For $i \leq(l-1)$, LHS $=\sum_{j=1, j \neq i}^{(l-1)} \mathbf{w}_{i}^{j}-\mathbf{u}_{l}^{i}$ $=\mathbf{w}_{i}-\mathbf{u}_{l}^{i}=\mathbf{u}_{i}$ (from the definition of $\mathbf{w}_{i}$ ). Finally, we can readily see that $\mathbf{u}_{i}^{j}=-\mathbf{u}_{j}^{i}, i=1, \ldots, l, j=1, \ldots, l, j \neq i$. This completes the induction step and the proof.

\section{Appendix D: Computation of Fractional Area $\boldsymbol{\beta}_{\mathrm{k}(\mathrm{c})}$ in Spatial Domain}

Consider the overlay of the halftone screens that constitutes $\mathbf{k}(\mathbf{c})$. Assume that $\boldsymbol{\Lambda}_{\mathbf{k}(\mathbf{c})}=\bigcap_{k \in \mathbf{k}(\mathbf{c})} \boldsymbol{\Lambda}_{k}$ is a 2D lattice, whose periodicity is given by $\mathbf{V}_{\mathbf{k}(\mathbf{c})}$ and unit cell by $\mathcal{U}_{\mathbf{k}(\mathbf{c})}$. First, we consider the representation of a single separation halftone screen in Eq. (3) and express this in alternate form as 
$h_{k}^{\left(\mathbf{d}_{k}\right)}(\mathbf{x})=\widetilde{s}_{k}\left(\mathbf{x}-\mathbf{d}_{k}\right) *\left(\sum_{\mathbf{n}_{k}} \delta\left(\mathbf{x}-\mathbf{V}_{\mathbf{k}(\mathbf{c})} \mathbf{n}_{k}\right)\right)$,

where the term $\widetilde{s}_{k}\left(\mathbf{x}-\mathbf{d}_{k}\right)$ includes repetitions of the halftone spot function of the $k^{\prime}$ th separation in $\mathcal{U}_{\mathbf{k}(\mathbf{c})}$ and can be written as

$\widetilde{s}_{k}\left(\mathbf{x}-\mathbf{d}_{k}\right)=s_{k}\left(\mathbf{x}-\mathbf{d}_{k}\right) *\left(\sum_{\mathbf{x}_{k} \in \boldsymbol{\Lambda}_{k} \cap \mathcal{U}_{\mathbf{k}(\mathbf{c})}} \delta\left(\mathbf{x}-\mathbf{x}_{k}\right)\right)$.

Using this convention, the Fourier transform of $h_{k}^{\left(\mathbf{d}_{k}\right)}(\mathbf{x})$ can be written as

$$
\begin{aligned}
H_{k}^{\left(\mathbf{d}_{k}\right)}(\mathbf{u})= & S_{k}(\mathbf{u}) \exp \left(-2 \pi j \mathbf{d}_{k}^{T} \mathbf{u}\right) \\
& \times\left(\sum_{\mathbf{x}_{k} \in \boldsymbol{\Lambda}_{k} \cap \mathcal{U}_{\mathbf{k}(\mathbf{c})}} \exp \left(-2 \pi j \mathbf{x}_{k}^{T} \mathbf{u}\right)\right) \\
& \times\left(\frac{1}{\left|\mathcal{U}_{\mathbf{k}(\mathbf{c})}\right|} \sum_{\mathbf{n}_{k}} \delta\left(\mathbf{u}-\mathbf{W}_{\mathbf{k}(\mathbf{c})} \mathbf{n}_{k}\right)\right),
\end{aligned}
$$

where $\mathbf{W}_{\mathbf{k}(\mathbf{c})}$ is the basis matrix for the lattice $\boldsymbol{\Lambda}_{\mathbf{k}(\mathbf{c})}^{*}$. Using this expression, Eq. (16) can be rewritten as

$$
\beta_{\mathbf{k}(\mathbf{c})}=\frac{1}{\mid \mathcal{U}_{\left.\mathbf{k}(\mathbf{c})\right|^{m}}} \sum_{\mathbf{n}_{k_{1}}, \ldots, \mathbf{n}_{k_{m}}}\left(\prod_{k \in \mathbf{k}(\mathbf{c})} S_{k}\left(\mathbf{W}_{\mathbf{k}(\mathbf{c})} \mathbf{n}_{k}\right) \exp \left(-2 \pi j \mathbf{d}_{k}^{T} \mathbf{W}_{\mathbf{k}(\mathbf{c})} \mathbf{n}_{k}\right)\left(\sum_{\mathbf{x}_{k} \in \Lambda_{k} \cap \mathcal{U}_{\mathbf{k}(\mathbf{c})}} \exp \left(-2 \pi j \mathbf{x}_{k}^{T} \mathbf{W}_{\mathbf{k}(\mathbf{c})} \mathbf{n}_{k}\right)\right) \delta\left(\mathbf{n}_{k_{1}}+\cdots+\mathbf{n}_{k_{m}}\right)\right)
$$

The set of indices satisfying $\mathbf{n}_{k_{1}}+\mathbf{n}_{k_{2}}+\cdots+\mathbf{n}_{k_{m}}=\mathbf{0}$ can equivalently be defined by the condition $\mathbf{n}_{k_{1}}=-\left(\mathbf{n}_{k_{2}}+\cdots+\mathbf{n}_{k_{m}}\right)$. Thus, the summation in Eq. (52) can be written as

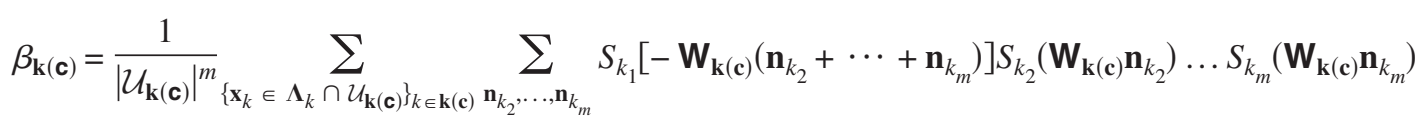

$$
\begin{aligned}
& \times \exp \left[-2 \pi j\left(\left(\mathbf{d}_{k_{2}}+\mathbf{x}_{k_{2}}-\mathbf{d}_{k_{1}}-\mathbf{x}_{k_{1}}\right)^{T} \mathbf{W}_{\mathbf{k}(\mathbf{c})} \mathbf{n}_{k_{2}}+\cdots+\left(\mathbf{d}_{k_{m}}+\mathbf{x}_{k_{m}}-\mathbf{d}_{k_{1}}-\mathbf{x}_{k_{1}}\right)^{T} \mathbf{W}_{\mathbf{k}(\mathbf{c})} \mathbf{n}_{k_{m}}\right)\right]
\end{aligned}
$$

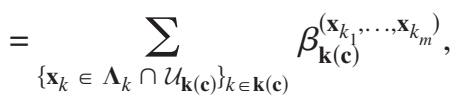

where

$$
\begin{aligned}
\beta_{\mathbf{k}(\mathbf{c})}^{\left(\mathbf{x}_{k_{1}}, \cdots, \mathbf{x}_{k_{m}}\right)}= & \frac{1}{\left|\mathcal{U}_{\mathbf{k}(\mathbf{c})}\right|^{m}} \int S_{k_{1}}\left(\mathbf{u}_{k_{1}}\right) \exp \left(-2 \pi j\left(\mathbf{d}_{k_{1}}+\mathbf{x}_{k_{1}}\right)^{T} \mathbf{u}_{k_{1}}\right)\left(S_{k_{2}}\left(\mathbf{u}_{k_{2}}\right) \exp \left(-2 \pi j\left(\mathbf{d}_{k_{2}}+\mathbf{x}_{k_{2}}\right)^{T} \mathbf{u}_{k_{2}}\right) \sum_{\mathbf{n}_{k_{2}}} \delta\left(\mathbf{u}_{k_{2}}-\mathbf{W}_{\mathbf{k}(\mathbf{c})} \mathbf{n}_{k_{2}}\right)\right) \cdots \\
& \left(S_{k_{m}}\left(\mathbf{u}_{k_{m}}\right) \exp \left(-2 \pi j\left(\mathbf{d}_{k_{m}}+\mathbf{x}_{k_{m}}\right)^{T} \mathbf{u}_{k_{m}}\right) \sum_{\mathbf{n}_{k_{m}}} \delta\left(\mathbf{u}_{k_{m}}-\mathbf{W}_{\mathbf{k}(\mathbf{c})} \mathbf{n}_{k_{m}}\right)\right) \delta\left(\mathbf{u}_{k_{1}}+\mathbf{u}_{k_{2}}+\cdots+\mathbf{u}_{k_{m}}\right) \mathrm{d} \mathbf{u}_{k_{1}} .
\end{aligned}
$$

Following Eq. (37), Eq. (54) can be modified such that it represents the value of a convolution integral at $\mathbf{u}=\mathbf{0}$ as

$$
\begin{aligned}
\beta_{\mathbf{k}(\mathbf{c})}^{\left(\mathbf{x}_{k_{1}}, \ldots, \mathbf{x}_{k_{m}}\right)=} & \frac{1}{\left|\mathcal{U}_{\mathbf{k}(\mathbf{c})}\right|^{m}} S_{k_{1}}(\mathbf{u}) \exp \left(-2 \pi j\left(\mathbf{d}_{k_{1}}+\mathbf{x}_{k_{1}}\right)^{T} \mathbf{u}\right) *\left(S_{k_{2}}(\mathbf{u}) \exp \left(-2 \pi j\left(\mathbf{d}_{k_{2}}+\mathbf{x}_{k_{2}}\right)^{T} \mathbf{u}\right)\right. \\
& \times\left.\sum_{\mathbf{n}_{k_{2}}} \delta\left(\mathbf{u}-\mathbf{W}_{\left.\left.\mathbf{k}(\mathbf{c}) \mathbf{n}_{k_{2}}\right)\right) * \cdots}\right) *\left(S_{k_{m}}(\mathbf{u}) \exp \left(-2 \pi j\left(\mathbf{d}_{k_{m}}+\mathbf{x}_{k_{m}}\right)^{T} \mathbf{u}\right) \sum_{\mathbf{n}_{k_{m}}} \delta\left(\mathbf{u}-\mathbf{W}_{\mathbf{k}(\mathbf{c}) \mathbf{n}_{k_{m}}}\right)\right)\right|_{\mathbf{u}=\mathbf{0}} .
\end{aligned}
$$

The value of the convolution integral at $\mathbf{u}=\mathbf{0}$ can be written as the spatial average of the inverse Fourier transform of the function as

$\beta_{\mathbf{k}(\mathbf{c})}^{\left(\mathbf{x}_{k_{1}}, \ldots, \mathbf{x}_{k_{m}}\right)}=\frac{1}{\left|\mathcal{U}_{\mathbf{k}(\mathbf{c})}\right|} \sum_{\mathbf{n}_{k_{2}}, \ldots, \mathbf{n}_{k_{m}}} \int s_{k_{1}}\left(\boldsymbol{\tau}-\mathbf{d}_{k_{1}}-\mathbf{x}_{k_{1}}\right) \prod_{k \in \mathbf{k}(\mathbf{c})\left\{k_{1}\right\}} s_{k}\left(\boldsymbol{\tau}-\mathbf{d}_{k}-\mathbf{x}_{k}-\mathbf{V}_{\mathbf{k}(\mathbf{c})} \mathbf{n}_{k}\right) \mathrm{d} \boldsymbol{\tau}$

If all displacement vectors are constrained to the unit cells of $\boldsymbol{\Lambda}_{k_{i}}+\boldsymbol{\Lambda}_{k_{j}}$, then it can be shown that these multiplications are zero unless the indices of the summation $\mathbf{n}_{k_{2}}, \ldots, \mathbf{n}_{k_{m}}$ take values from the set $\{-1,0,1\}$. Similar to Eq. (37), this function is modified such that it represents the value of a function at $\mathbf{x}=\mathbf{0}$ as 


$$
\begin{aligned}
\beta_{\mathbf{k}(\mathbf{c})}^{\left(\mathbf{x}_{\left.k_{1}, \ldots, \mathbf{x}_{k_{m}}\right)}\right.} & =\left.\frac{1}{\left|\mathcal{U} \mathcal{U}_{\mathbf{k}(\mathbf{c})}\right|} \sum_{\mathbf{n}_{k_{2}}, \ldots, \mathbf{n}_{k_{m}}} \int s_{k_{1}}\left(\boldsymbol{\tau}-\mathbf{d}_{k_{1}}-\mathbf{x}_{k_{1}}\right) \prod_{k \in \mathbf{k}(\mathbf{c})\left\{\left\{k_{1}\right\}\right.} s_{k}\left(\boldsymbol{\tau}-\mathbf{d}_{k}-\mathbf{x}_{k}-\mathbf{V}_{\mathbf{k}(\mathbf{c})} \mathbf{n}_{k}-\mathbf{x}\right) \mathrm{d} \boldsymbol{\tau}\right|_{\mathbf{x}=\mathbf{0}} \\
& =\left.\frac{1}{|\mathcal{U} \mathbf{k}(\mathbf{c})|} \sum_{\mathbf{n}_{k_{2}}, \ldots, \mathbf{n}_{k_{m}}} s_{k_{1}}\left(\mathbf{x}-\mathbf{d}_{k_{1}}-\mathbf{x}_{k_{1}}\right) *\left(\prod_{k \in \mathbf{k}(\mathbf{c})\left\{k_{1}\right\}} s_{k}\left(-\mathbf{x}-\mathbf{d}_{k}-\mathbf{x}_{k}-\mathbf{V}_{\mathbf{k}(\mathbf{c})} \mathbf{n}_{k}\right)\right)\right|_{\mathbf{x}=\mathbf{0}}
\end{aligned}
$$

Then, $\beta_{\mathbf{k}(\mathbf{c})}$ can be computed following Eq. (53) as

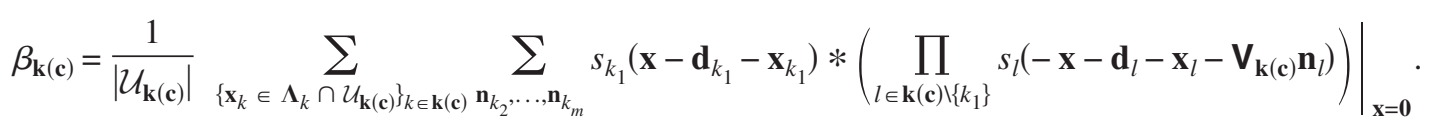

This equation can be equivalently written as that

$$
\begin{aligned}
& \beta_{\mathbf{k}(\mathbf{c})}=\left.\frac{1}{\left|\mathcal{U}_{\mathbf{k}(\mathbf{c})}\right|} \sum_{\left\{\mathbf{x}_{k} \in \boldsymbol{\Lambda}_{k} \cap \mathcal{U}_{\mathbf{k}(\mathbf{c})\}}\right.} k \in \mathbf{k}(\mathbf{c}) \sum_{\mathbf{n}_{k_{2}}, \ldots, \mathbf{n}_{k_{m}}} s_{k_{1}}\left(\mathbf{x}-\mathbf{d}_{k_{1}}\right) *\left(\prod_{l \in \mathbf{k}(\mathbf{c})\left\{k_{1}\right\}} s_{l}\left[-\mathbf{x}-\mathbf{d}_{l}-\left(\mathbf{x}_{l}-\mathbf{x}_{k_{1}}\right)-\mathbf{V}_{\mathbf{k}(\mathbf{c})} \mathbf{n}_{l}\right]\right)\right|_{\mathbf{x}=\mathbf{0}},
\end{aligned}
$$

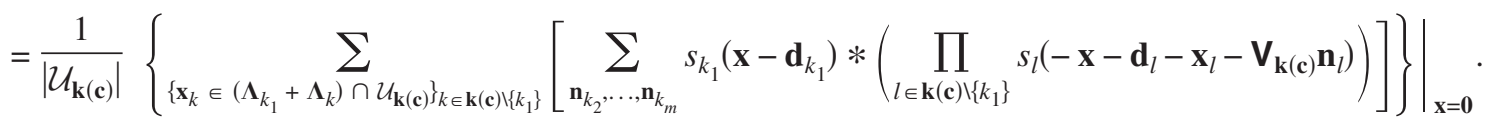

\section{Appendix E: List of Symbols}

$K=$ Number of colorant separations in the contone/halftone image

$k=$ Index of the $k^{\prime}$ th colorant separation of the contone/halftone image

$\mathbf{x}=$ Vector of 2D spatial coordinates $\mathbf{x}$ $=[x, y]^{T}$

$h_{k}(\mathbf{x})=$ Halftone separation of the $k$ th colorant separation of the contone image

$\boldsymbol{\Lambda}_{k}=$ Periodicity lattice for the $k^{\prime} t h$ halftone separation

$\mathbf{V}_{k}=$ Basis matrix for the lattice $\boldsymbol{\Lambda}_{k}$

$\mathbf{n}_{k}=$ Coordinate index for point $\mathbf{V}_{k} \mathbf{n}_{k}$ in the lattice $\boldsymbol{\Lambda}_{k}$

$\mathcal{U}_{k}=$ Unit cell of the lattice $\boldsymbol{\Lambda}_{k}$

$s_{k}(\mathbf{x})=$ Halftone spot function of the $k^{\prime}$ th halftone separation

$\beta_{k}=$ Fractional area covered by the $k^{\prime}$ th halftone separation

$\mathbf{d}_{k}=$ Displacement misregistration of the $k^{\prime} t h$ halftone separation

$\lambda=$ Optical wavelength

$\gamma=$ Yule-Nielsen correction factor

$\mathbf{c}=K$-bit binary index string $c_{1} \cdots c_{K}$ representing a Neugebauer primary, where the binary value $c_{k}$ indicates presence/ absence of the $k^{\prime}$ th colorant in the Neugebauer primary

$R_{\mathbf{c}}(\lambda)=$ Reflective spectrum of the Neugebauer primary c

$a_{\mathbf{c}}=$ Fractional area covered by the Neugebauer primary $\mathbf{c}$

$\mathbf{k}(\mathbf{c})=k_{1} \cdots k_{m}$ string of colorant indices for which $c_{k_{l}}$ is non-zero

$h_{\mathbf{k}(\mathbf{c})}(\mathbf{x})=$ Halftone overlay obtained by the superposition of the halftone separations that constitute $\mathbf{k}(\mathbf{c})$

$$
\begin{aligned}
& \Lambda_{\mathbf{k}(\mathbf{c})}=\text { Periodicity lattice for the halftone over- } \\
& \mathbf{V}_{\mathbf{k}(\mathbf{c})}=\text { Basis matrix for the lattice } \boldsymbol{\Lambda}_{\mathbf{k}(\mathbf{c})} \\
& \mathcal{U}_{\mathbf{k}(\mathbf{c})}=\text { Unit cell of the lattice } \Lambda_{\mathbf{k}(\mathbf{c})} \\
& \beta_{\mathbf{k}(\mathbf{c})}=\text { Fractional area covered by the overlay } \\
& \mathbf{u}=\text { Vector of } 2 \mathrm{D} \text { frequency coordinates } \mathbf{u} \\
& =[u, v]^{T} \\
& H_{k}(\mathbf{u})=\text { Fourier transform of the halftone sepa- } \\
& \text { ration } h_{k}(\mathbf{x}) \\
& \Lambda_{k}^{*}=\text { Reciprocal lattice of the lattice } \boldsymbol{\Lambda}_{k} \\
& \mathbf{W}_{k}=\text { Basis matrix for the lattice } \Lambda_{k}^{*} \\
& S_{k}(\mathbf{u})=\text { Fourier transform of the halftone spot } \\
& \text { function } s_{k}(\mathbf{x}) \\
& H_{\mathbf{k}(\mathbf{c})}(\mathbf{u})=\text { Fourier transform of the halftone over- } \\
& \text { lay } h_{\mathbf{k}(\mathbf{c})}(\mathbf{x})
\end{aligned}
$$

\section{Acknowledgment}

This work was supported in part by an award from the Xerox Foundation and by a grant from New York State Office of Science, Technology, and Academic Research (NYSTAR) through the Center for Electronic Imaging Systems (CEIS). We would like to thank Dr. Wencheng Wu of Xerox Corporation, Webster, New York for providing measurements of interseparation displacement misregistration for our printed targets. We would also like to thank two of the anonymous reviewers for their extensive comments and suggestions that have helped improve this paper.

\section{References}

1. B. Oztan, G. Sharma, and R. P. Loce, "Quantitative evaluation of misregistration induced color shifts in color halftones," in Color Imaging X: Processing, Hardcopy, and Applications, R. Eschbach and G. G. Marcu, Eds., Proc. SPIE 5667 501-512 (2005). 
2. B. Oztan, G. Sharma, and R. P. Loce, "Analysis of misregistration induced color shifts in the superposition of periodic screens," in Color Imaging XI: Processing, Hardcopy, and Applications, R. Eschbach and G. G. Marcu, Eds., Proc. SPIE 6058, 60580X1 (2006)

3. B. Oztan, G. Sharma, and R. P. Loce, "Conditions for color misregistration sensitivity in clustered-dot halftones," in Proc. IEEE Intl. Conf. Image Proc., 4, 221-224 (2007).

4. C. M. Hains, S. Wang, and K. T. Knox, "Digital color halftones," Chapter 6, in Digital Color Imaging Handbook, G. Sharma, Ed., CRC Press, Boca Raton, Fla. (2003).

5. F. A. Baqai, J.-H. Lee, A. U. Agar, and J. P. Allebach, "Digital color halftoning," IEEE Signal Process. Mag. 22, 87-96 (2005).

6. J. A. C. Yule, Principles of Color Reproduction, Applied to Photomechanical Reproduction, Color Photography, and the Ink, Paper, and Other Related Industries, Wiley, New York (1967)

7. G. Sharma, "Color fundamentals for digital imaging," Chapter 1, in Digital Color Imaging Handbook, G. Sharma, Eds., CRC Press, Boca Raton, Fla. (2003).

8. R. N. Bracewell, The Fourier Transform and Its Applications, 2 ed., McGraw-Hill, New York (1986)

9. W. H. F. Talbot, "Improvements in the art of engraving," British Patent Specification No. 565 (1852)

10. H. E. J. Neugebauer, "Die theoretischen Grundlagen des Mehrfarbenbuchdrucks," Zeitschrift für wissenschaftliche Photographie Photophysik und Photochemie 36, 73-89 (1937); Reprinted in Neugebauer Memorial Seminar on Color Reproduction, K. Sayangi, Ed., Proc. SPIE 1184, 194-202 (1989).

11. J. A. S. Viggiano, "Modeling the color of multi-colored halftones," Proc.-TAGA pp. 44-62 (1990).

12. E. Demichel, in Procédé 26, 17-21 (1924); ibid 26, 26-27 (1924)

13. R. Balasubramanian, "A printer model for dot-on-dot halftone screens," in Color Hard Copy and Graphic Arts IV, J. Bares, Ed., Proc. SPIE 2413, 356-364 (1995).

14. R. D. Hersch and A. K. Singla, "An ink spreading model for dot-ondot spectral prediction," in Proc. IS\&T/SID 14th Color Imag. Conf. 38-43 (2006).

15. G. L. Rogers, "Neugebauer revisited: random dots in halftone screening," Color Res. Appl. 23, 104-113 (1998).

16. I. Amidror and R. D. Hersch, "Neugebauer and Demichel: dependence and independence in n-screen superpositions for colour printing," Color Res. Appl. 25, 267-277 (2000)

17. I. Amidror and R. D. Hersch, "Analysis of the microstructures ("rosettes') in the superposition of periodic layers," J. Electron. Imaging 11, 316-337 (2002)

18. J. Chen, "An investigation of color variation as a function of register in dot-on-dot multicolor halftone printing," in Proc.-TAGA pp. 315 334 (1984).

19. K. Daels and P. Delabastita, "Color balance in conventional halftoning," in Proc.-TAGA pp. 1-18 (1994).

20. T. S. Rao and G. R. Arce, "Halftone patterns for arbitrary screen periodicities," J. Opt. Soc. Am. A 5, 1502-1511 (1988).

21. T. S. Rao, G. R. Arce, and J. P. Allebach, "Analysis of ordered dither for arbitrary sampling lattices and screen periodicities," IEEE Trans. Acoust., Speech, Signal Process. 38, 1981-2000 (1990).

22. J. W. S. Cassels, An Introduction to the Geometry of Numbers, 1st ed., Springer, New York (1959).

23. J. A. C. Yule and W. J. Neilsen [sic], "The penetration of light into paper and its effect on halftone reproduction," in Proc.-TAGA pp. 65-76 (1951).

24. F. R. Ruckdeschel and O. G. Hauser, "Yule-Nielsen effect in printing: a physical analysis," Appl. Opt. 17, 3376-3383 (1978).

25. M. Pearson, " $\mathrm{n}$ value for general conditions," in Proc.-TAGA 32 415-425 (1980)

26. D. R. Wyble and R. S. Berns, "A critical review of spectral models applied to binary color printing," Color Res. Appl. 25, 4-19 (2000).

27. C. C. MacDuffee, The Theory of Matrices, Chelsea, New York (1946).

28. E. Dubois, "The sampling and reconstruction of time-varying imagery with application in video systems," Proc. IEEE 73, 502-522 (1985)

29. C. S. Iliopoulos, "Worst-case complexity bounds on algorithms for computing the canonical structure of finite abelian groups and the hermite and smith normal forms of an integer matrix," SIAM J. Com put. 18(4), 658-669 (1989).

30. CIE, "Colorimetry." CIE Publication No. 15.2, Central Bureau of the CIE, Vienna (1986). The commonly used data on color matching functions is available at the CIE Web site at http://www cie.co.at/.

31. R. J. Pellar and L. Green, "Electronic halftone generator," U.S. Patent No. 4,149,183 (1979).

32. R. J. Pellar, "Electronic halftone generator," U.S. Patent No. 4,196,151 (1980)

33. I. E. Sutherland and G. W. Hodgman, "Reentrant polygon clipping," Commun. ACM 17, 32-42 (1974).

34. M. Xia, E. Saber, G. Sharma, and A. M. Tekalp, "End-to-end color printer calibration by total least squares regression," IEEE Trans. Image Process. 8, 700-716 (1999).
35. W. L. Rhodes and C. M. Hains, "The influence of halftone orientation on color gamut and registration sensitivity," in Proc. IS\&T's 46th Annu. Conf., pp. 180-182 (1993).

36. D. N. Curry, "Two dimensional linearity and registration error correction in a hyperacurity printer," U.S. Patent No. 5,732,162 (1998).

37. F. P. Gauthier and D. L. Jovic, "Image registration method," U.S Patent No. 5,760,914 (1998)

38. R. P. Loce, M. E. Banton, and S. J. Harrington, "System for electronic compensation of beam scan trajectory distortion," U.S. Patent No. 6,529,643 (2003)

39. D. R. Rasmussen, E. N. Dalal, and S. J. Zoltner, "Technique for accurate color-color registration measurements," U.S. Patent No. 6,529,616 (2003)

40. S. Gustavson, "Color gamut of halftone reproduction," J. Imaging Sci. Technol. 41, 283-290 (1997).

41. R. D. Hersch and F. Crété, "Improving the Yule-Nielsen modified spectral Neugebauer model by dot surface coverages depending on the ink superposition conditions," in Color Imaging X: Processing, Hardcopy, and Applications, R. Eschbach and G. G. Marcu, Eds., Proc. SPIE 5667, 434-445 (2005).

42. B. Lawler, The Complete Guide to Trapping, 2 ed., Hayden Books, Indianapolis, IN (1995).

43. F. Baqai and J. Allebach, "Computer-aided design of clustered-dot color screens based on a human visual system model," Proc. IEEE 90, 104-122 (2002)

44. S. Wang, "Uniform rosette for moiré free color halftoning," in Color Imaging XII: Processing, Hardcopy, and Applications, R. Eschbach and G. G. Marcu, Eds., Proc. SPIE 6493, 64931E (2007).

45. T. Chen and P. P. Vaidyanathan, "The role of integer matrices in multidimensional multirate systems," IEEE Signal Process. Mag. 41(3), 1035-1047 (1993)

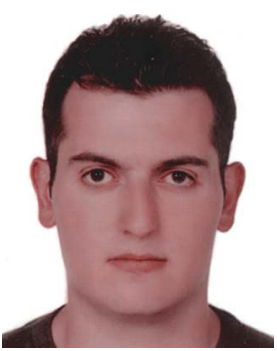

Basak Oztan received his BS (with high honors) in electrical and electronics engineering from Middle East Technical University, Ankara, Turkey, in 2003; his MS in electrical and computer engineering from the University of Rochester, Rochester, New York, in 2004. He is currently pursuing a $\mathrm{PhD}$ in the Department of Electrical and Computer Engineering, University of Rochester, Rochester, New York. He was a summer intern at the Xerox Webster Research Center, Webster, New York, during the summers of 2005 and 2006. He received a student paper award at International Conference on Acoustics, Speech, and Signal Processing (IEEE) 2006 in image and multidimensional signal processing category. His research interests include color imaging, color halftoning, and watermarking for printed media. He is a student member of SPIE, IS\&T, IEEE, and the IEEE Signal Processing Society.

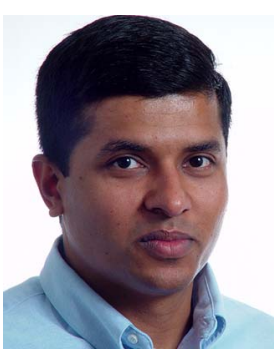

Gaurav Sharma received his BE in electronics and communication engineering from Indian Institute of Technology Roorkee (formerly University of Roorkee), India, in 1990; his ME in electrical communication engineering from the Indian Institute of Science, Bangalore, India, in 1992; and his MS in applied mathematics and PhD in electrical and computer engineering (ECE) from North Carolina State University (NCSU), Raleigh, in 1995 and 1996, respectively. From August 1992 through August 1996, he was a research assistant at the Center for Advanced Computing and Communications in the ECE Department at NCSU. From August 1996 through August 2003, he was with Xerox Research and Technology, in Webster, New York, initially as a member of research staff and subsequently at the position of principal scientist. Since the fall of 2003, he has been an associate professor at the University of Rochester in the Department of Electrical and Computer Engineering and in the Department of Biostatistics and Computational Biology. His research interests include color science and imaging, multimedia security and watermarking, and genomic signal processing. $\mathrm{He}$ is the editor of the Color Imaging Handbook, published by CRC press in 2003. He is a member of IS\&T; a senior member of the IEEE; and a member of Sigma Xi, Phi Kappa Phi, and Pi Mu Epsilon honor societies. He currently serves as an associate editor for the Journal of Electronic Imaging, IEEE Transactions on Image Processing, and IEEE Transactions on Information Forensics and Security. 


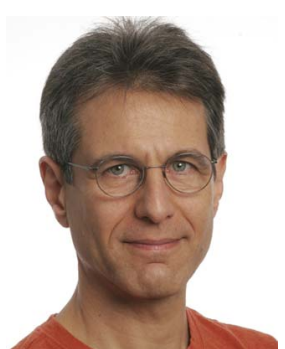

Robert P. Loce is a principal scientist at the Xerox Research Center, Webster, New York. He joined Xerox in 1981 with an associate degree in optical engineering technology from Monroe Community College. While working in the Optical and Imaging Technology and Research departments at Xerox, he received his BS in photographic science from the Rochester Institute of Technology (RIT) in 1985, his MS in optical engineering from the University of Rochester in 1987, and PhD in imaging science from RIT in 1993. His current work involves development of image processing methods for color electronic printing. He has publications and many patents in the areas of halftoning, digital image rendering, optics, imaging systems, and digital image enhancement. His publications include book chapters on digital halftoning and digital document processing, and a book on enhancement and restoration of digital documents. He is a fellow of SPIE and member of IEEE. He is currently an associate editor for Journal of Electronic Imaging and has been an associate editor for Real-Time Imaging and IEEE Transactions on Image Processing. 